\title{
Copyright
}

by

Amin Ettehadtavakkol

2009 
The Thesis Committee for Amin Ettehadtavakkol

Certifies that this is the approved version of the following thesis:

\section{Gas Storage Facility Design Under Uncertainty}

\section{APPROVED BY SUPERVISING COMMITTEE:}

Supervisor:

Christopher J. Jablonowski

Larry W. Lake 


\title{
Gas Storage Facility Design Under Uncertainty
}

\author{
by
}

\section{Amin Ettehadtavakkol, BS.}

\author{
Thesis \\ Presented to the Faculty of the Graduate School of \\ The University of Texas at Austin \\ in Partial Fulfillment of the Requirements for the Degree of
}

Master of Science in Engineering

The University of Texas at Austin

December 2009 


\section{Dedication}

To my parents 


\section{Acknowledgements}

I would like to express my sincere appreciation for guidance and encouragement provided by my advisor, Dr. Christopher J. Jablonowski. The full and hearty support of Dr. Larry W. Lake is gratefully acknowledged. I also appreciate the unconditional support of my parents, Mostafa, and Noori, my brothers and my sister.

December 2009 


\title{
Abstract \\ Gas Storage Facility Design Under Uncertainty
}

\author{
Amin Ettehadtavakkol, MSE \\ The University of Texas at Austin, 2009
}

Supervisor:

Christopher J. Jablonowski

In the screening and concept selection stages of gas storage projects, many estimates are required to value competing projects and development concepts. These estimates are important because they influence which projects are selected and which concept proceeds into detailed engineering. In most cases, there is uncertainty in all of the estimates. As a result, operators are faced with the complex problem of determining the optimal design. A systematic uncertainty analysis can help operators solve this problem and make better decisions. Ideally, the uncertainty analysis is comprehensive and includes all uncertain variables, and simultaneously accounts for reservoir behavior, facility options, and economic objectives. This thesis proposes and demonstrates a workflow and an integrated optimization model for uncertainty analysis in gas storage. The optimization model is fast-solving and eliminates most constraints on the scope of the uncertainty analysis. Using this or similar workflows and models should facilitate analysis and communication of results within the project team and with other stakeholders. 


\section{Table of Contents}

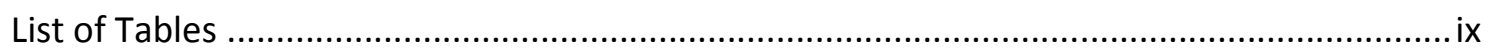

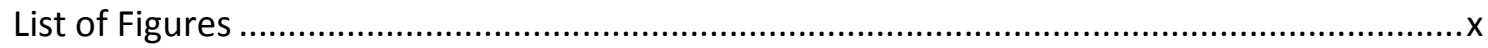

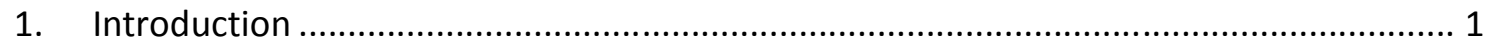

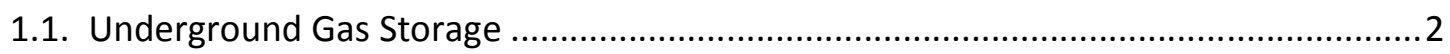

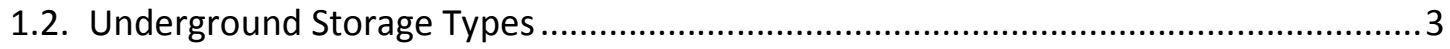

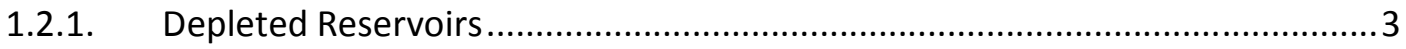

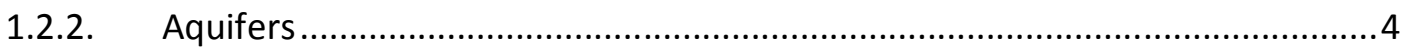

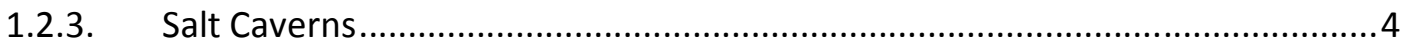

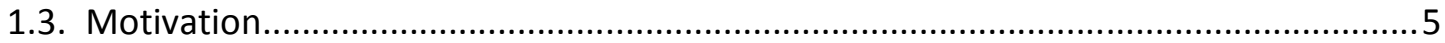

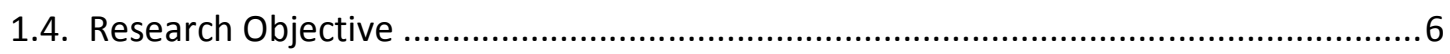

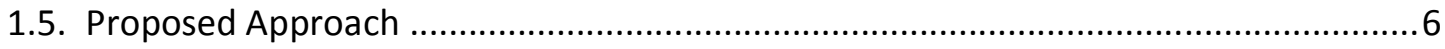

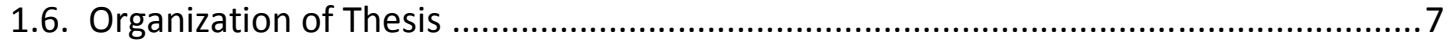

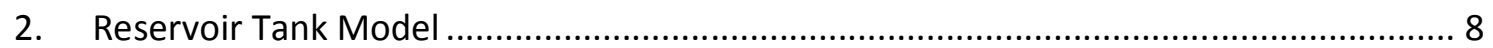

2.1. Compressible Liquid Tank Model....................................................................... 10

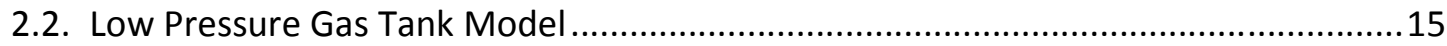

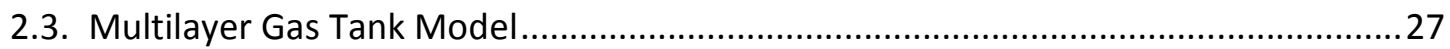

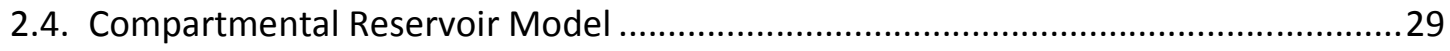

2.5. Multi Layer - Multi Compartment Reservoir ............................................................ 34

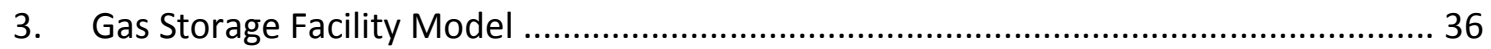

3.1. Literature Review on Gas Storage Facility Design and Planning ................................38

3.2. Selected Approach to Problem Definition ................................................................. 41

3.3. Basic Definitions for Gas Storage Facility ............................................................... 42

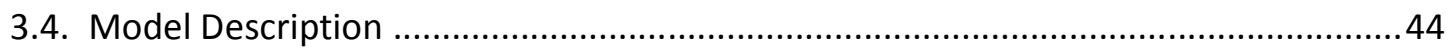

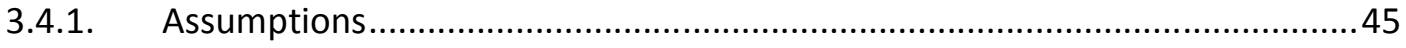

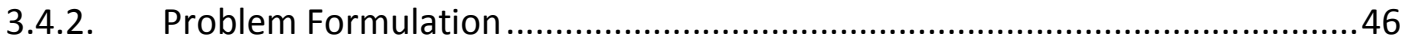

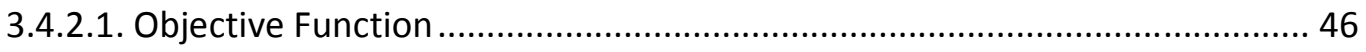

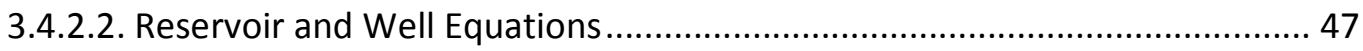

3.4.2.3. Compression and Associated Horsepower Requirements ........................... 48 


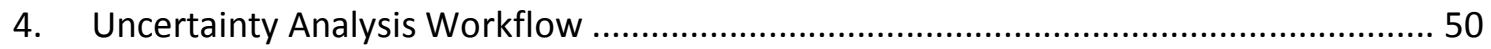

4.1. Step 1: Specify the Functionality Requirements....................................................51

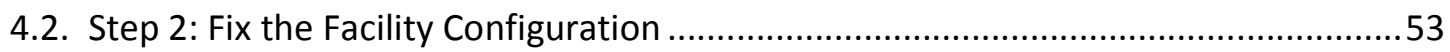

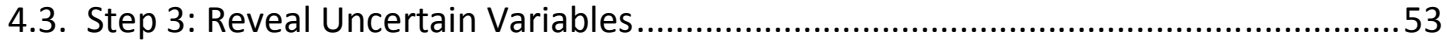

4.4. Step 4: Minimize the Total Cost for the Facility ........................................................53

4.5. Step 5: Create the Probability Density Function for the Total Cost ............................54

4.6. Step 6: Obtain the Optimal Configuration ..................................................................54

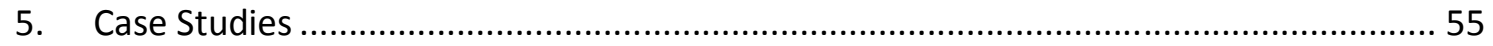

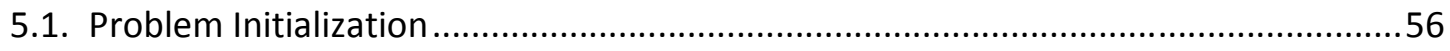

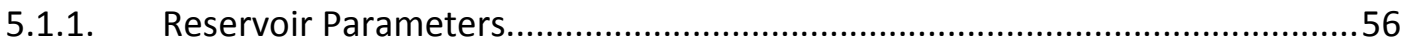

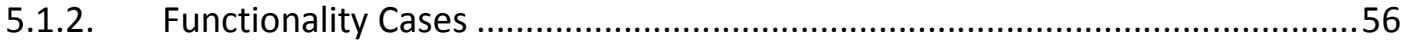

5.1.3. Constants, Parameters, Variables and Decision Variables .............................57

5.2. Asset-based problem: Deterministic Profit Maximization .......................................... 57

5.2.1. Analysis of the Compression Horsepower .................................................. 62

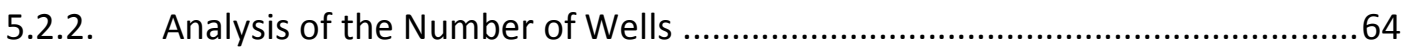

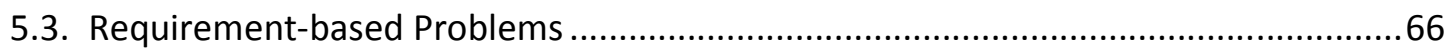

5.3.1. Problem A: Deterministic Analysis for Functionality Requirements ..............66

5.3.2. Problem B: Uncertainty Analysis for Functionality Requirements .................70

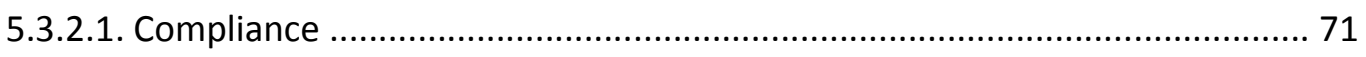

5.3.3. Problem C: Uncertainty Analysis for Reservoir Properties ..............................74

5.3.3.1. Sensitivity Analysis of Model Outputs …..................................................... 75

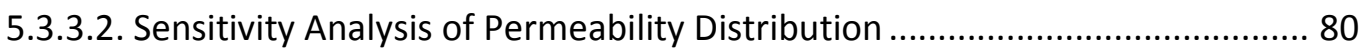

5.3.4. Problem D: Joint Uncertainty in Permeability \& Functionality Requirements 81

6. Summary, Conclusions and Recommendations for Future Work ................................. 86

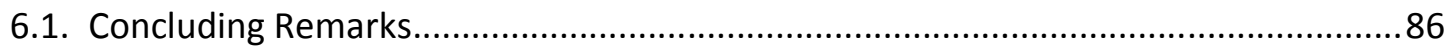

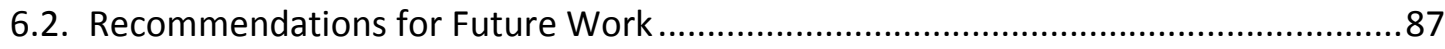

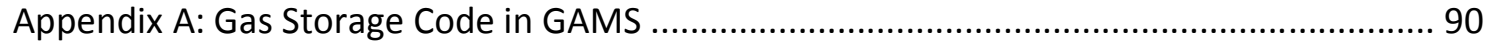

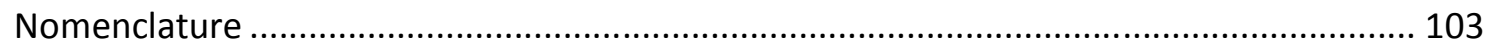

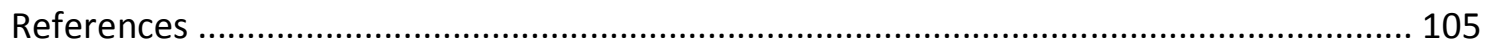

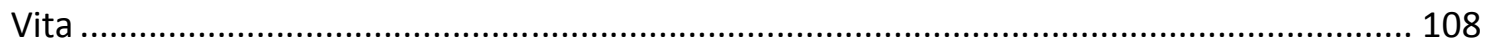




\section{List of Tables}

Table 2-1: Producer/Injector parameters ......................................................................... 18

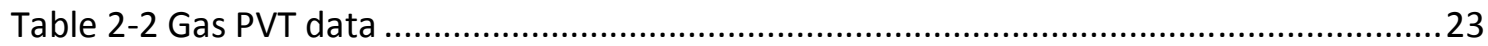

Table 2-3 Summary of Equations for dry gas tank model at low pressure ............................26

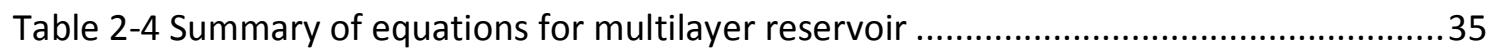

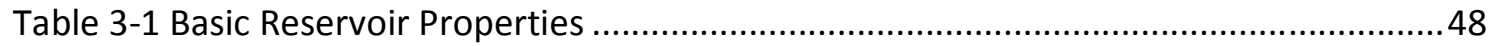

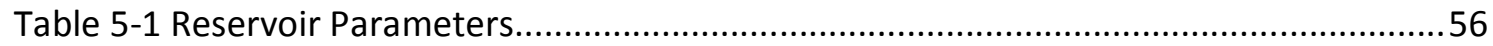

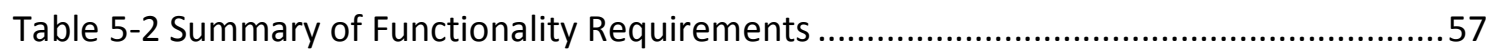

Table 5-3. Comparison between cost minimization and profit maximization problems ........59

Table 5-4: Economic characteristics of the diagnostic case ..............................................59

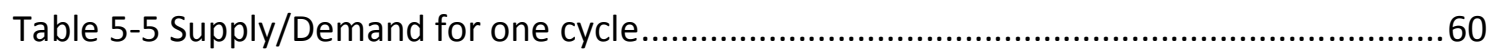

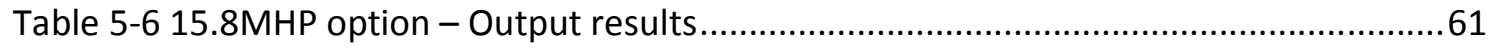

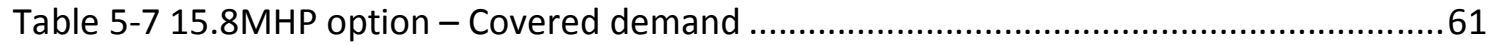

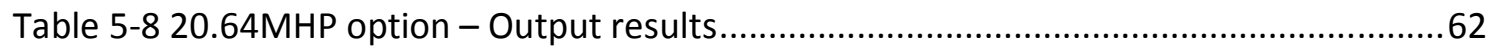

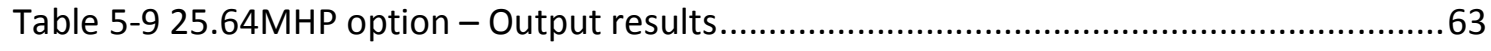

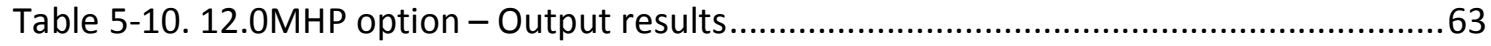

Table 5-11 Statistical parameters for 25.64MHP and different well counts .........................71

Table 5-12 Permeability Stats - All Observations............................................................... 76

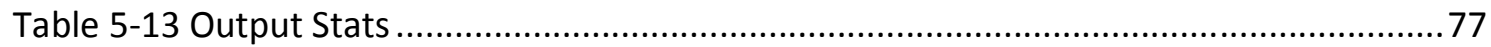




\section{List of Figures}

Figure 2-1 Linear relation between the $\mathrm{P} / \mathrm{Z}$ and flow rates......................................22

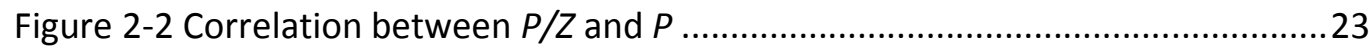

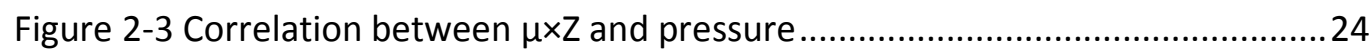

Figure 3-1 General annual trend for market demand .............................................36

Figure 4-1 Uncertainty Analysis Workflow ............................................................. 51

Figure 5-1 Relative NPV value for configuration of 32 wells ...................................64

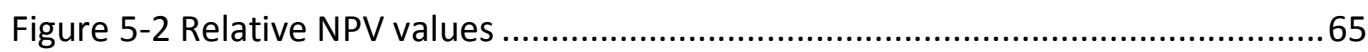

Figure 5-3 Relative NPV values: around the optimal point .......................................65

Figure 5-4 Cost Curves for Functionality Case 1 .......................................................67

Figure 5-5 Cost Curves for Functionality Case 2 .................................................67

Figure 5-6 Optimal Base Gas for Functionality Case 1..............................................69

Figure 5-7 Optimal Base Gas for Functionality Case 2 ............................................69

Figure 5-8 PDF of CAPEX/Working Gas for Functionality Case 2 ...............................70

Figure 5-9 of Working Gas for Functionality Case 2 .................................................. 71

Figure 5-10 Cost and Compliance Curves for Functionality Case 2 …........................73

Figure 5-11 Cost and Compliance Curves for Functionality Case 2: Multiple HP ........73

Figure 5-12 Cost Curves and Compliance ............................................................ 74

Figure 5-13 CAPEX/WG - Permeability Correlation - 45 Wells....................................76

Figure 5-14 Base gas - Permeability Correlation - 45 Wells ........................................78

Figure 5-15 Cost Curves and Compliance for Three Permeability Distributions ..........81

Figure 5-16 Cost Curves and Compliance for Joint Uncertainty Analysis ....................83 


\section{Introduction}

Demand for natural gas is seasonal, and gas storage facilities are used to balance supply and demand. Natural gas storage facilities employ underground geologic formations as the storage medium. The type of geologic formation selected for the storage facility determines the performance characteristics and constraints of the storage facility (its functionality), and thus the application of the storage asset. Likewise, when the functionality is specified first, it dictates the type of geologic formation that must be acquired.

Gas storage facilities are typically located close to demand centers, and most employ depleted oil or gas reservoirs as the storage medium. ${ }^{1}$ The reservoirs are often high permeability and lie in intermediate depths (2000-5000 ft) with normal temperature gradients. The reservoirs do not bear mobile aqueous phase or mobile oleic phase (Bennion et al., 2000). A reservoir is good gas storage candidate if it has sufficient permeability to allow production and injection at high rates in peak periods. It should also have sufficient pore volume and structural closure. The major components of a reservoir-based gas storage facility include the reservoir, horizontal and/or vertical wells, gathering lines and associated flow controls, and dehydration and compression equipment. One may also consider the base gas (the quantity of gas intended as permanent inventory) as part of the facility because it requires a significant capital expenditure akin to the surface facilities and wells. More detailed information on the technology, economics, and regulation of the gas storage industry can be found in $\operatorname{FERC}(2004)$ and $\operatorname{EIA}(2004,2006)$.

\footnotetext{
1 Gas is also stored in salt caverns and aquifers.
} 


\subsection{Underground Gas Storage}

Gas storage provides gas supply when current production is inadequate to meet the demand. Gas storage has existed since the early 20th Century, traditionally serving the purpose of meeting seasonal load variations and being utilized in emergency situations. However, because of recent unbundling of gas storage and other regulatory shifts, natural gas storage applications have broadened. Storage is utilized to:

- Meet regulatory obligations to maintain a reliable supply of gas at the lowest cost to the ratepayer.

- Prevent shippers from incurring imbalance penalties, in addition to facilitating daily nomination changes, 'park and loan' services, and simultaneous injection and withdrawals.

- Mitigate price volatility by ensuring commodity liquidity at gas market centers.

- Offset the tapered reduction in traditional supplies used to meet winter demand.

- Levelize production by providing an outlet for producers to store gas when it is not immediately marketable.

- Act as a tool for marketers to capitalize on speculation and arbitrage.

- Balance volume delivery integrity during summer demand spikes due to gasfired electric generation. ${ }^{2}$

Many of the preceding applications for natural gas storage were enabled by regulatory changes, although the economic benefits and financial opportunities are the primary drivers in the development of natural gas projects. The financial viability of a storage asset is strongly influenced by the type of geologic formation selected

\footnotetext{
${ }^{2}$ Federal Energy Regulatory Commission 2004, p. 4.
} 
for the storage facility, the market demand and the storage costs. This provides the basic motivation for investigating the appropriate configuration for a storage facility.

\subsection{Underground Storage Types}

There are three types of underground storage: depleted reservoirs, aquifers and salt caverns. Depleted reservoirs are investigated in detail in this thesis. However, a brief description of all types is presented in this section.

\subsubsection{Depleted Reservoirs}

Depleted oil and gas reservoirs are the most common geologic formations used in natural gas storage. These formations are reservoirs that have already been depleted of all economically recoverable hydrocarbons. Depleted reservoirs are proven and secure containers of hydrocarbons, having usually contained them for millennia.

There are several advantages to using reservoirs that have been previously explored and produced. One advantage is that the geologic characteristics of the formation have already been determined through exploration, production and history matching. Wells, gathering lines, and other extractive equipment left inplace can be re-utilized for storage withdrawal. Furthermore, depleted reservoirs already contain economically unrecoverable hydrocarbon deposits and thus do not require the injection of physically unrecoverable gas. Although, in some instances gas injected is enriched by the residual hydrocarbons. The increased heating content of the gas can necessitate further processing upon withdrawal if the gas's heating value exceeds the limit for "pipeline quality" standards. All of these advantages yield tangible cost savings in the development of gas storage facilities. On average a, depleted reservoir requires approximately $50 \%$ of its working gas capacity to be allocated for base gas. These reservoirs are typically evaluated by the financial ramifications of their geologic and geographic characteristics (NaturalGas.org, 2009).

Geologically, reservoir formations with high porosity and high permeability are ideal. A reservoir's porosity dictates the volume of gas it can store, while its 
permeability indicates its deliverability and injectivity. Depleted oil and gas reservoirs typically have the largest storage capacities. However, they are not characterized as having particularly high deliverability or injectivity, in comparison to other storage media. Geographically, reservoirs near a market center or transportation infrastructure are economically attractive (NaturalGas.org, 2009).

\subsubsection{Aquifers}

Aquifers are porous permeable underground formations that are occupied by water. The quality of water occupying aquifers varies from fresh to virtually brinesaturated. It is possible to re-condition and modify these formations for the storage of natural gas, although they are more expensive to develop than depleted reservoirs (NaturalGas.org, 2009).

A significant amount of resources, in the form of time and capital, are invested in geological exploration of an aquifer. The physical parameters of the formation must be determined in order to assess whether a formation is technically capable of storing gas and is economically viable. Significant resources must also be invested in the development of storage infrastructure. Wells, extraction equipment, compression equipment, and pipelines are required. Powerful injection equipment is needed to meet the pressure requirements to displace the aquifer's resident water with natural gas. Gas extracted from an aquifer has to be re-processed and

dehydrated in order to meet "pipeline quality" standards. Additionally, aquifers lack the gas retention capabilities of depleted reservoirs; therefore special 'collector wells' must be installed to capture injected gas that would otherwise escape the primary aquifer formation (NaturalGas.org, 2009).

\subsubsection{Salt Caverns}

Salt caverns are storage facilities formed out of underground salt deposits. These salt deposits exist in the form of salt domes or salt beds. Salt domes are the thicker of the two formations. Salt domes used for gas storage typically have depths of 6,000 to 1,500 feet beneath the surface. Salt beds are thinner, yet wider than salt 
domes. They typically have thicknesses that do not exceed 1,000 feet. Comparatively, salt beds are more expensive to develop into storage facilities and more prone to deterioration than salt domes (NaturalGas.org, 2009).

In order to convert these salt formations into salt caverns suitable for gas storage, a process known as leaching is implemented. Wells are drilled into the salt formation and high volumes of water are cycled through the formation. The water

dissolves and extracts a portion of the salt deposited; essentially creating a cavernous void within the formation that gas can be stored in. The process of leaching and the installation of the requisite extraction equipment represent vast development costs. Of all three geologic formations, salt caverns require the largest amount of capital investment (NaturalGas.org, 2009).

\subsection{Motivation}

During the screening and concept selection stages of gas storage projects, many estimates are required to value competing projects and development concepts. These estimates determine which projects are selected and which concept proceeds into front end engineering and design. Estimates are required for reservoir properties, well performance, capital costs, operating costs, facility performance, construction schedule, and market demand and price. The importance of these estimates is self-evident, they determine which projects go forward, the configuration and sizing of facilities, project timing, and ultimately, the value derived from the project.

In most cases, there is uncertainty in all of the estimates used in screening and concept selection. As a result, operators are faced with the complex problem of developing a design that is robust to various revelations of the uncertain variables. A systematic uncertainty analysis can help operators solve this problem by describing and quantifying the sensitivity of project value to the design decisions and to the uncertainties. Use of the information from the uncertainty analysis should result in better decisions. Ideally, an uncertainty analysis is comprehensive and includes all 
uncertain variables, and simultaneously accounts for reservoir and production behavior, facility options, and economic objectives.

The investment costs also influence how the storage asset is utilized. Natural gas storage projects are substantial investments; development costs for storage projects can range from $\$ 5$ - $\$ 25$ million per billions of cubic feet (Bcf) depending on the size and type of facility (FERC, 2004).

\subsection{Research Objective}

The goal of the present investigation is to propose and demonstrate a workflow and integrated model for uncertainty analysis in gas storage facility design. To achieve this goal an integrated optimization model of the reservoir, facilities and market is developed. To perform the uncertainty analysis a systematic framework is proposed. Using the model along with the workflow can help to analyze the trade offs and make better facility design decisions.

\subsection{Proposed Approach}

To achieve the research objectives, the following approach is adopted:

1. Define and explain the basics of gas storage reservoirs and their key uncertainties

2. Define and explain the concepts of the tank reservoir model

3. Develop the gas storage optimization model based on the features of gas storage projects and the gas tank model equations

4. Propose a workflow for uncertainty analysis

5. Apply the workflow to the gas storage optimization model to solve problems. The concepts of optimal configuration, consistency and feasibility are presented and analyzed. The examples will illustrate the potentials of the proposed workflow to reveal the effects of uncertainty on the optimal configuration 


\subsection{Organization of Thesis}

Chapter 2 discusses the tank model and the reasons to select the tank model. The equations of a gas tank reservoir model are developed. The model is also extended to describe vertical and horizontal heterogeneity. The purpose of this chapter is to provide the required equations for describing the reservoir behavior. Obviously, no model is perfect; however, based on the purpose there is a level of descriptive detail that would suffice to capture the key features. Chapter 3 is specifically devoted to the formulation of the gas storage model in the form of a mathematical programming problem. Chapter 4 provides a detailed description of the workflow and its components. The purpose is to apply the workflow to the mathematical model of gas storage and to analyze the effects of those uncertainties on the optimal configuration. Chapter 5 presents the results of stylized cost minimization and profit maximization problems. Determinist, probabilistic and joint uncertainty problems are analyzed and compared. The stylized problems illustrate the potentials of the proposed systematic framework to analyze the trade offs and make better facility design decisions. 


\section{Reservoir Tank Model}

The purpose of the current chapter is to provide a model for describing the reservoir behavior. As we will see, the main challenge of selecting a reservoir model is to balance the accuracy against the level of detail included and the imposed computational effort. The equations and correlations presented in this chapter are included in the integrated gas storage model.

The tank, zero-dimensional or homogeneous model is the basic building block of any reservoir system (Odeh, 1969). The tank model is a mathematical representation of hydrocarbon production (Walsh and Lake, 2003). Like with any other model, the tank model entails simplifications and assumptions (Hultzsch and lake, 2007). It assumes that average properties are sufficient to represent the reservoir. The tank model can be used to estimate the pressure and production behavior of homogeneous to weakly heterogeneous formations where the variance of the permeability field assumed lognormal is small (Coats, 1969; Jankovic, Fiori and Dagan, 2006).

We start with the low pressure gas tank model which is relatively simple and can be applied to low to moderate-pressure gas reservoirs. Afterwards we discuss the multilayer gas tank model, and present a simple example to show how to develop the equations for a dry gas reservoir. The equations derived in this section form a basis to simulate a gas storage reservoir, which is the subject of steps III and IV.

There is a substantial literature that discusses the advantages and disadvantages of the tank models against the detailed, fine-grid reservoir models. To summarize the discussions, supporters of fine-grid models commonly argue that for 
most reservoirs that are inherently heterogeneous, the gross-simplification of reservoir geology with a tank model is not a valid alternative to detailed modeling. They cite the limited applicability of tank models to multiphase systems and spatial or localized reservoir features. However, the use of fine-grid reservoir models is not admissible in screening and exploratory cases because the data, especially data to populate a detailed model, is scarce and uncertain.

The most important result of these discussions - and perhaps the main principle of any engineering practice - is that the use of model should be based on the application (Coats, 1969, Lake 2007). In light of this fact, the following reasons justify the use of tank model for the gas storage facility design:

1. The tank model is a simple and yet, powerful and sufficient tool for screening. It represents the reservoir by simple analytical equations which are appropriate to be coupled with economical equations for screening a gas storage project. Here the purpose is to gather all these parameters, decision variables and constraints to form a robust framework for decision analysis under uncertainty.

2. In planning stage of storage reservoir development, simple reservoir models and optimization models are preferred over complex reservoir simulators because of their simplicity and sufficiency to capture the key features of reservoir behavior. They can be used to interpret performance and optimal operating and investment plans, while complex reservoir simulators require much more time and may not be cost effective (McVay and Spivey, 2001).

3. When the uncertainty in costs, supply and demand and the subsurface parameters are accounted for, the optimization procedure becomes complicated so that it might not be feasible to run complex simulator to compare thousands of possible decisions and find the optimal solution to the problem.

4. The availability of data for assigning the properties in fine-grid block models is an important issue in complex simulators. Coats (1969) noted that the use 
of sophisticated fine grid simulators with limited available input data might lead to unrealistic results. Odeh $(1969,1982)$ has reminded that the appropriate choice of flow simulator should depend primarily on the application.

5. Finally, this research is not intended to provide exact numerical and analytical solutions for low pressure gas reservoir; the purpose of using a reservoir model is to describe the behavior of a gas reservoir with simple and applicable equations.

In this chapter, we present different versions of the tank model. We start with the compressible liquid tank model which is relatively simple and it can be applied to oil reservoirs as well as moderate to high-pressure gas reservoirs. Afterwards we discuss the low-pressure gas tank model, and present a simple example to show how to develop the equations for a dry gas reservoir. Finally, we present the extended version of the tank model to account for multiple layers as well as multiple blocks with transmissibility factors.

\subsection{Compressible Liquid Tank Model}

The compressible liquid tank model simulates the depletion performance of reservoir containing a single compressible fluid. This model not only applies to oil, but also to many moderate and high-pressure gases. The flow is from a single layer. This model can identify the key variables affecting recovery, and establishes a time scale for recovery.

The model implies some important predictions, including:

1. In the compressible liquid tank model pressure will decline exponentially with time.

2. The producing rate is proportional to permeability, pay thickness, and the number of producing wells, if the effect of interference between the pay zones is neglected. It is inversely proportional to the viscosity. 
3. The producing rate will decline at a constant rate; this implies that the rate will decline exponentially with time. The decline rate is equal to a decay constant, which is proportional to the permeability, pay thickness and the number of wells and inversely proportional to reservoir pore volume and fluid compressibility.

4. The time to realize a given rate is inversely proportional to the decay constant.

The volume of the fluid recovered down to a certain average pressure is proportional to the reservoir pore volume and compressibility.

This model assumes a homogeneous reservoir; however this assumption can be relaxed to describe a stratified reservoir. In these cases, the effect of layering significantly affects performance.

The compressible-liquid tank model is based on the following idealizations:

1. The reservoir can be treated as a homogeneous block (one value for each property) and that the single phase flow comes from a single layer.

2. There are at most three fluid components: stock-tank oil, surface gas, and stock-tank water.

3. There are at most, two fluid phases present at reservoir conditions: oleic and aqueous.

4. Stock-tank water does not partition into the oleic phase.

5. Stock-tank oil does not partition into the aqueous phase.

6. The aqueous phase is immobile

The surface oil producing rate from well $\mathrm{k}$ is related to the average reservoir pressure through the following equation:

$q_{o s c, k}=J_{k} \frac{\bar{p}-p_{w f, k}}{B_{o}}$ 
Where:

$q_{o s c, k}: \quad$ Surface rate from well $k, S T B$

$\bar{p}: \quad$ Average reservoir pressure, $p s i$

$p_{w f, k}: \quad$ flowing bottomhole pressure for well $k, p s i$

$J_{k}: \quad$ Productivity index(PI) for well $k, b b l / S T B / D a y$

$B_{o}: \quad$ Formation volume factor, $b$ bl/STB

Equation 2-1 is called as deliverability equation and it assumes semi-steady state flow. The deliverability equation not only applies to liquids whose viscosity and compressibility are constant but also to any fluid whose $1 / \mu B$ or $\rho / \mu$ is approximately linear with pressure or whose $P / \mu z$ is approximately constant. This equivalency applies to most gases over the pressure of 2500 to 3000 psia. So, the equation is appropriate for high-pressure gas reservoir (i.e. $\bar{p} \geq 3000$ psia ).

Applying the Darcy law for single phase flow for steady state or semi steady state flow of a slightly compressible fluid or high pressure gas with constant viscosity, gives:

$$
J_{k}=\frac{0.00708 k h}{\mu_{g}\left(\frac{1}{2} \ln \frac{A}{r_{w, k}^{2} C_{A, k}+5.75+s_{k}}\right)}
$$

Where:

$J_{k}$ : Productivity index for well $\mathrm{k}$, bbl/psi/day

$k$ : $\quad$ Permeability, $m d$

$h: \quad$ Total thickness, $f t$

$\mu_{g}: \quad$ Viscosity, $\mathrm{cp}$

A: $\quad$ Area, acres

$C_{A, k}:$ Shape factor

$s_{k}: \quad$ Skin factor

Applying the mathematical manipulations on the macroscopic equations for oil phase at standard conditions, the material balance equation (MBE) will be:

$$
q_{o s c, k}=-\frac{V_{p} C_{t}}{B_{o}} \frac{d \bar{P}}{d t}
$$


The field producing rate is the sum of individual well rates:

$q_{o s c}=\sum_{k=1}^{N_{w}} q_{o s c, k}=\sum_{k=1}^{N_{w}} J_{k} \frac{\bar{p}-p_{w f, k}}{B_{o}}$

Equating 2-3 with 2-4 yields:

$-\frac{V_{p} C_{t}}{B_{o}} \frac{d \bar{P}}{d t}=\sum_{k=1}^{N_{w}} J_{k} \frac{\bar{p}-p_{w f, k}}{B_{o}}$

Separating variables and setting the limits of integration,

$\int_{p_{i}}^{\bar{p}} \frac{d \bar{p}}{\sum_{k=1}^{N_{w}} J_{k}\left(\bar{p}-p_{w f, k}\right)}=-\frac{1}{V_{p} C_{t}} \int_{0}^{t} d t$

Integrating and evaluating the integration limits gives

$$
\frac{\sum_{k=1}^{N_{w}} J_{k}\left(\bar{p}-p_{w f, k}\right)}{\sum_{k=1}^{N_{w}} J_{k}\left(p_{i}-p_{w f, k}\right)}=\exp (-\lambda t)
$$

Where $\lambda$ is called the decay constant and is equal to:

$$
\lambda=\frac{\sum_{k=1}^{N_{w}} J_{k}}{V_{p} C_{t}}
$$

$\lambda$ has units of reciprocal time (1/day). The integration assumes $V_{p}$ and $C_{t}$ as constants. For the special case of wells with identical productivity indices and bottomhole pressures:

$$
\begin{aligned}
& \frac{\bar{p}-p_{w f, k}}{p_{i}-p_{w f, k}}=\exp (-\lambda t) \\
& \lambda=\frac{N_{w} J}{V_{p} C_{t}}
\end{aligned}
$$


Equation 2-10 shows that the pressure declines exponentially; which is a noticeable observation. Substituting in the deliverability equation and solving for $q_{\text {osc }}$ gives

$$
q_{o s c}=\frac{\exp (-\lambda t)}{\bar{B}_{o}} \sum_{k=1}^{N_{w}} J_{k}\left(p_{i}-p_{w f, k}\right)
$$

Setting $t=0$, for initial condition:

$$
q_{o s c i}=\frac{1}{\bar{B}_{g}} \sum_{k=1}^{N_{w}} J_{k}\left(p_{i}-p_{w f, k}\right)
$$

Dividing the two equations, we obtain:

$$
q_{o s c}=q_{o s c i} \exp (-\lambda t)
$$

Equation 2-13 shows that the production rate declines exponentially. This is the characteristic feature of the primary depletion of slightly compressible fluids, or gases at high pressure during the depletion flow. The gas produced during a certain period of time can be easily found through integration:

$$
N_{p}=\int_{0}^{t} q_{o s c} d t=\int_{0}^{t} q_{o s c i} \exp (-\lambda t) d t
$$

Integrating and evaluating the limits of integration:

$$
N_{p}=\frac{q_{o s c i}}{\lambda}[1-\exp (-\lambda t)]=\frac{q_{o s c i}-q_{o s c}}{\lambda}
$$

Note that

$$
\exp (-\lambda t)=\frac{q_{o s c}}{q_{o s c i}}
$$

The compressible liquid tank model provides the general understanding of the way in which reservoir behavior is described; however, for the case of gas storage reservoirs a low pressure gas tank model is more appropriate, and it is slightly different from the compressible liquid model. 


\subsection{Low Pressure Gas Tank Model}

The compressible liquid tank model not only applies to liquids, but also applies to gases if pressures are greater than 2500psi to 3000psi (Walsh and Lake, 2003). So there is a need to develop a tank model specifically for low pressure gases. The new model assumes the fluid's $\mu z$ product is approximately constant. This idealization usually applies to any gas up to pressures of approximately 2000psi. If the pressure is below 200 to 300psi then the formulas for the ideal gas can also be applied. Together, the compressible liquid model and the low-pressure gas tank models provide a more complete analysis of gas reservoir performance.

There are two methods to derive the equations for low pressure gas reservoirs, and both are based on the material balance equation. The first method derives an equation for the average reservoir pressure i.e. $\bar{P}$. The second method derives an equation for the average pressure-compressibility ratio, i.e. $\overline{\left(\frac{P}{Z}\right)}$. In both methods, the equations derived cannot be solved analytically without major simplifying assumptions (Walsh and Lake, 2003).

If the pressure is above 2500 psia, the equation for average reservoir pressure does not change, having the assumptions for the tank model:

$$
\bar{V}_{p} c_{t} \frac{d \bar{P}}{d t}=\sum_{k=1}^{N_{i n j}} i_{k}(t)-\sum_{k=1}^{N_{p r o}} q_{k}(t)
$$

Note that the total compressibility is shown by:

$$
\begin{aligned}
& c_{t}=\bar{S}_{o} c_{o}+\bar{S}_{w} c_{w}+\bar{S}_{g} c_{g}+c_{f} \\
& c_{t}=\bar{S}_{o} B_{o} \frac{\partial\left(1 / B_{o}\right)}{\partial P}+\bar{S}_{w} B_{w} \frac{\partial\left(1 / B_{w}\right)}{\partial P}+\bar{S}_{g} B_{g} \frac{\partial\left(1 / B_{g}\right)}{\partial P}+\frac{1}{V_{p}} \frac{\partial V_{p}}{\partial P}
\end{aligned}
$$

Assuming single phase gas flow, we have: 


$$
c_{t}=\bar{S}_{w} B_{w} \frac{\partial\left(1 / B_{w}\right)}{\partial P}+\bar{S}_{g} B_{g} \frac{\partial\left(1 / B_{g}\right)}{\partial P}+\frac{1}{V_{p}} \frac{\partial V_{p}}{\partial P}
$$

If we ignore the water compressibility and rock compressibility (which are invariably negligible compared to gas compressibility), then the deliverability equation for the surface-gas component yields:

$$
V_{p} \bar{S}_{g} \frac{\partial\left(1 / B_{g}\right)}{\partial P} \frac{d \bar{P}}{d t}=-\left(q_{g s c}(t)-i_{g s c}(t)\right)
$$

Where $\mathrm{q}_{\mathrm{gsc}}$ is the standard producing rate of gas and $\mathrm{i}_{\mathrm{gsc}}$ is the standard injection rate of gas. This equation assumes the pore volume and gas saturation are constant. Gas formation volume factor for real gas is

$$
B_{g}=z T p_{s c} / T_{s c} p
$$

It follows that

$$
\frac{\partial 1 / B_{g}}{\partial p}=\frac{T_{s c}}{z T p_{s c}}
$$

In Equation 2-22, we have assumed that $z$ is constant, substituting for $\frac{\partial / B_{g}}{\partial p}$ in the Equation 2-20, gives

$$
V_{p}\left(1-\bar{S}_{w i}\right) \frac{d \bar{P}}{d t}=-\frac{\left(q_{g s c}(t)-i_{g s c}(t)\right) z T p_{s c}}{T_{s c}}
$$

The deliverability equation for a flowing low pressure gas well is

$$
q_{g s c, k}=J_{k}\left(\bar{P}^{2}-P_{w f, k}^{2}\right)^{n}
$$

$q_{g s c, k}$ is the surface-gas rate from well $k$ and $J_{k}$ is the productivity index for well $k$. The exponent $n$ is included to account for the laminar and turbulent flow. For laminar flow (low permeability rock), $n=1$; for the turbulent flow (high permeability 
rock), $n=0.5$. In practice the best value for $n$ is 0.7 . There are analytical methods to determine the exponent $n$ (Ahmed and McKinney, 2005). This equation is sometimes called the back pressure equation and $n$ is called the back pressure exponent. For real gas semi-steady state laminar flow, $J_{k}$ is given by

$$
J_{k}=\frac{\pi h k T_{s c}}{p_{s c} T\left(\mu_{g} z\right)_{a v g}\left(\frac{1}{2} \ln \frac{4 A}{r_{w, k}^{2} e^{\gamma} C_{A}}+s_{k}\right)}=\frac{0.703 h k}{T\left(\mu_{g} z\right)_{a v g}\left(\frac{1}{2} \ln \frac{A}{r_{w, k}^{2} C_{A}}+5.75+s_{k}\right)}
$$

The right expression assumes the oil field units, where

$$
\begin{array}{ll}
J_{k}: & \text { Productivity index for well } \mathrm{k}, s c f / p s i^{2} / \text { day } \\
k: & \text { Permeability, } m d \\
h: & \text { Total thickness for well } \mathrm{k}, f t \\
\mu_{g}: & \text { Gas viscosity, } c p \\
Z: & \text { Gas deviation factor } \\
T: & \text { Temperature, }{ }^{o} R \\
A: & \text { Area, acres } \\
C_{A}: & \text { Shape factor } \\
r_{w, k}: & \text { Wellbore radius, } f t \\
s_{k}: & \text { Skin factor }
\end{array}
$$

The productivity index equation (2-25) can also be written in the following form as:

$$
J_{k}=\frac{k h_{k}}{1422\left(\mu_{g} z\right)_{\text {avg }} T\left(\frac{1}{2} \ln \left(\frac{4 A}{1.781 C_{A} r_{w, k}^{2}}\right)+s_{k}\right)}
$$

In which $J_{k}[=] \frac{M s c f}{d a y \times p s i^{2}}$ and $A[=] \mathrm{ft}^{2}$. The latter form of the productivity index equation is more appropriate for the purpose gas storage model. Summary of the parameters for productivity index and the values used for the reservoir model is presented in Table 2-1. 
Table 2-1 Producer/Injector parameters

\begin{tabular}{|c|l|c|}
\hline Parameter & \multicolumn{1}{|c|}{ Description } & Value \\
\hline$h$ & Reservoir thickness & $20 \mathrm{ft}$ \\
\hline$k$ & Permeability & $50 \mathrm{md}$ \\
\hline$T$ & Reservoir Temperature & $600^{\circ} \mathrm{R}$ \\
\hline$A$ & Drainage area of the wells & $30-60 \mathrm{acres}$ \\
\hline$R_{w}$ & Wellbore radius & $0.25 \mathrm{ft}$ \\
\hline$S$ & Skin factor & -4.0 \\
\hline$P_{w f, m i n}$ & Minimum well flowing pressure & $150 \mathrm{psi}$ \\
\hline$P_{\text {inj,max }}$ & Well injection pressure & $2600 \mathrm{psi}$ \\
\hline$(\mu \times Z)_{a v g}$ & Average for $\mu \times \mathrm{Z}$ at $\mathrm{P}_{\mathrm{wf}, \min }$ & $0.01345 \mathrm{cp}$ \\
\hline$(\mu \times \mathrm{Z})_{\text {inj,avg }}$ & Average for $\mu \times \mathrm{Z}$ at $\mathrm{P}_{\text {inj,max }}$ & $0.01386 \mathrm{cp}$ \\
\hline
\end{tabular}

Total field production is the sum of production from individual wells:

$Q_{p r o, t o t}=\sum_{k=1}^{N_{w}} q_{g s c, k}=\sum_{k=1}^{N_{w}} J_{p r o, k}\left(\bar{P}^{2}-P_{w f, k}^{2}\right)^{n}$

$N_{w}$ is the total number of producing wells.

Total field injection rate is the sum of injections to individual wells:

$Q_{i n j, t o t}=\sum_{k=1}^{N_{i n j}} i_{g s c, k}=\sum_{k=1}^{N_{i n j}} J_{i n j, k}\left(P_{i n j, k}^{2}-\bar{P}^{2}\right)^{n}$

$N_{i n j}$ is the total number of injection wells

If we substitute the total production and injection equations into the average pressure equation we have:

$$
V_{p}\left(1-\bar{S}_{w i}\right) \frac{d \bar{P}}{d t}=-\frac{z T p_{s c}}{T_{s c}}\left[\sum_{k=1}^{N_{w}} J_{p r o, k}\left(\bar{P}^{2}-P_{w f, k}^{2}\right)^{n}-\sum_{k=1}^{N_{i n j}} J_{i n j, k}\left(P_{i n j, k}^{2}-\bar{P}^{2}\right)^{n}\right]
$$

Equation 2-29 cannot be solved using analytical methods. However, if the correlation between the average pressure and the gas deviation factor is determined and the injection and bottomhole pressures are available, the equation can be 
numerically solved to find the average reservoir pressure. Also if the production or injection rates can be determined using an independent method, such as an optimization method, the equation can be solved to find the average pressure.

Before we proceed to the second method, let us reformulate Equation 2-29 in a more familiar form. The pore volume $V_{p}$ can be substituted by the equivalent initial gas in place $G B_{g i}$ as follows:

$$
G B_{g i} \frac{d \overline{\left(\frac{P}{Z}\right)}}{d t}=-\frac{T p_{s c}}{T_{s c}}\left[Q_{p r o, t o t}(t)-Q_{i n j, t o t}(t)\right]
$$

And using the equation for $\mathrm{B}_{\mathrm{gi}}\left(B_{g i}=\frac{T p_{s c}}{T_{s c}} \overline{\left(\frac{P}{Z}\right)_{i}^{-1}}\right)$, we can simplify Equation 230 as:

$$
\frac{d \overline{\left(\frac{P}{Z}\right)}}{d t}=-\overline{\left(\frac{P}{Z}\right)} \frac{1}{G}\left[Q_{p r o, t o t}(t)-Q_{i n j, t o t}(t)\right]
$$

Integrating this equation gives:

$d \overline{\left(\frac{P}{Z}\right)}=-\overline{\left(\frac{P}{Z}\right)} \frac{1}{G} \int_{0}^{t}\left(Q_{p r o, t o t}(t)-Q_{i n j, t o t}(t)\right) d t+C$

Assuming $\overline{\left(\frac{P}{Z}\right)}$ to be equal to $\overline{\left(\frac{P}{Z}\right)_{i}}$ at the initial time:

$\overline{\left(\frac{P}{Z}\right)}=\overline{\left(\frac{P_{i}}{Z_{i}}\right)}\left(1-\frac{G_{p}(t)-G_{I}(t)}{G}\right)$

$G_{p}(t)$ is the cumulative production and $G_{I}(t)$ is the cumulative injection.

The second method, which is common in the reservoir engineering textbooks, is the direct application of material balance equation for a dry gas reservoir: 
$\left(G_{p}-G_{I}\right) B_{g}+\left(W_{p}-W_{I}\right) B_{w}=G\left(B_{g}-B_{g i}\right)+G B_{g i} \frac{c_{w} S_{w i}+c_{f}}{1-S_{w i}} \Delta P+W_{e}$

In which:

$G_{p}:$ Cumulative gas production, Mscf

$G_{I}:$ Cumulative gas injection, Mscf

$G$ : Original gas in place, Mscf

$W_{p}$ : Cumulative water produced, STB

$W_{I}:$ Cumulative water injected, STB

$W_{e}$ : Cumulative water influx, $\mathrm{RB}$

$B_{g}$ : Gas formation volume factor, RB/Mscf

$B_{g i}$ : Initial gas formation volume factor, RB/Mscf

$B_{w}$ : Water formation volume factor, RB/STB

$c_{w}:$ Water compressibility factor $\mathrm{psi}^{-1}$

$c_{f}:$ Formation compressibility factor $\mathrm{psi}^{-1}$

$S_{w i}:$ Irreducible water saturation

$\Delta P:$ Reservoir pressure change, $\mathrm{psi}$

Assuming no water influx, no water production and no water injection and also neglecting the formation and the connate water expansion, which are negligible compared to the gas expansion, we have:

$$
\left(G_{p}-G_{I}\right) B_{g}=G\left(B_{g}-B_{g i}\right)
$$

$B_{g}$ is a function of pressure, temperature and gas deviation factor. The reservoir is assumed to be isothermal. Substituting the definition for $B_{g}$ into the equation above and simplifying the terms, this equation can be rephrased in terms of average pressure as:

$$
\overline{\left(\frac{P}{Z}\right)}=\overline{\left(\frac{P_{i}}{Z_{i}}\right)}\left(1-\frac{G_{p}(t)-G_{I}(t)}{G}\right)
$$

Which is exactly the same equation as derived before. If we take the derivative with respect to time, we have 


$$
\frac{d \overline{\left(\frac{P}{Z}\right)}}{d t}=-\overline{\left(\frac{P}{Z}\right)} \frac{1}{G}\left[Q_{p r o, t o t}(t)-Q_{i n j, t o t}(t)\right]
$$

If we write the equation in the discrete form, using a backward scheme, we will have:

$$
\overline{\left(\frac{P}{Z}\right)_{t}}-\overline{\left(\frac{P}{Z}\right)_{t-1}}=-\overline{\left(\frac{P}{Z}\right)_{i}} \frac{1}{G}\left[Q_{p r o, t o t}(t)-Q_{i n j, t o t}(t)\right]
$$

We can also derive the same equations using the cumulative form of the pressure equation. If we write the equation in the discrete form for two successive time periods, we will have:

$$
\begin{aligned}
& \overline{\left(\frac{P}{Z}\right)_{t}}=\overline{\left(\frac{P}{Z}\right)_{i}}\left(1-\frac{G_{p r o}(t)-G_{i n j}(t)}{G}\right) \\
& \overline{\left(\frac{P}{Z}\right)_{t-1}}=\overline{\left(\frac{P}{Z}\right)_{i}}\left(1-\frac{G_{p r o}(t-1)-G_{i n j}(t-1)}{G}\right)
\end{aligned}
$$

In which $G_{p r o}(t)$ and $G_{i n j}(t)$ are the cumulative production and injection rates respectively. We should keep the consistency of units to transform the daily rates to the cumulative rates. For example, if we choose the time step to be one month, we have:

$$
\begin{aligned}
& G_{p r o}(t)=G_{p r o}(t-1)+30 \times \sum_{k=1}^{N_{p r o}} q_{k}(t) \\
& G_{i n j}(t)=G_{i n j}(t-1)+30 \times \sum_{k=1}^{N_{p r o}} i_{k}(t)
\end{aligned}
$$

Now, if we subtract the two equations, we get the recursive equation representing the relation between the average pressure and the production/injection history. 


$$
\overline{\left(\frac{P}{Z}\right)_{t}}=\overline{\left(\frac{P}{Z}\right)_{t-1}}-\overline{\left(\frac{P}{Z_{i}}\right)} \frac{1}{G}\left[\left(G_{p}(t)-G_{p}(t-1)\right)-\left(G_{I}(t)-G_{I}(t-1)\right)\right]
$$

One of the important implications of Equation $2-43$ is that the $P / Z$ ratio is linearly related to the flow rates (see Figure 2-1). This version of the $\mathrm{MBE}^{3}$ is used in the optimization code (See Appendix A).

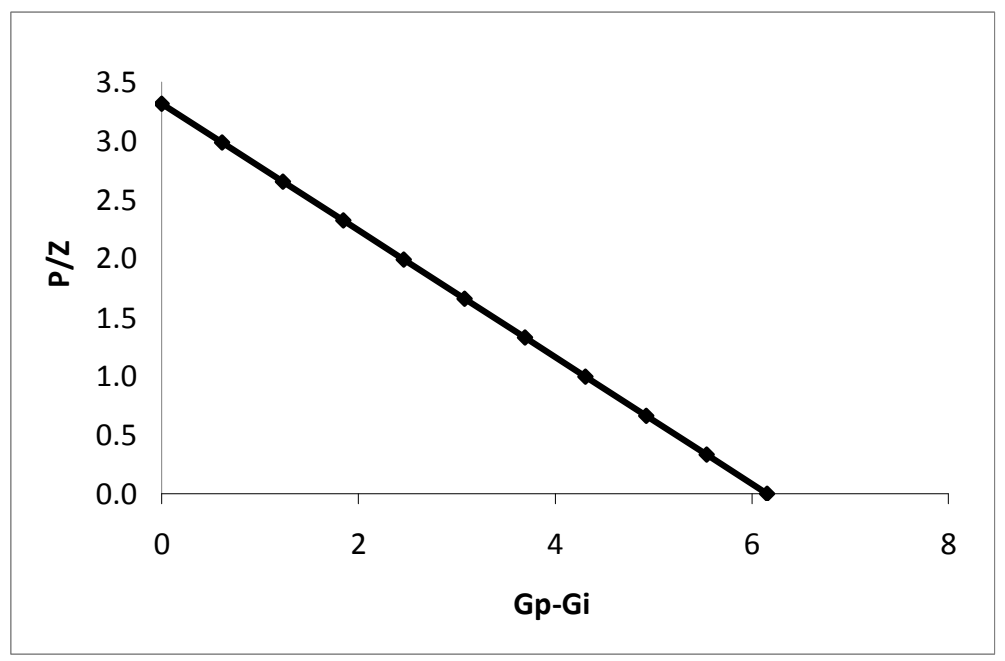

Figure 2-1 Linear relation between the $\mathrm{P} / \mathrm{Z}$ and flow rates (scaled)

Next, we must set up a relation between the average pressure and the $\overline{\left(\frac{P}{Z}\right)}$ ratio. To show the calculations, let us assume that the following PVT data apply to a dry gas reservoir:

\footnotetext{
${ }^{3}$ Material Balance Equation
} 
Table 2-2 Gas PVT data

\begin{tabular}{|c|c|c|c|}
\hline $\begin{array}{c}\text { Pressure } \\
(p s i)\end{array}$ & $\begin{array}{c}\text { Viscosity } \\
(c p)\end{array}$ & $\begin{array}{c}\text { Deviation } \\
\text { factor }\end{array}$ & $\begin{array}{c}\mathrm{B}_{\mathrm{g}} \\
\left(f \mathrm{t}^{3} / \mathrm{S} c f\right)\end{array}$ \\
\hline 0 & 0.013 & 1.000 & - \\
\hline 400 & 0.013 & 0.937 & 0.0397 \\
\hline 1200 & 0.015 & 0.832 & 0.0118 \\
\hline 1600 & 0.017 & 0.794 & 0.0084 \\
\hline 2000 & 0.018 & 0.770 & 0.0065 \\
\hline 3200 & 0.023 & 0.797 & 0.0042 \\
\hline 3600 & 0.025 & 0.827 & 0.0039 \\
\hline 4000 & 0.027 & 0.860 & 0.0036 \\
\hline
\end{tabular}

We are interested in finding the correlation between the pressure and the $P / Z$ ratio. We can simply use the data to set up a correlation:

$$
\overline{\left(\frac{P}{Z}\right)_{t}}=-5 \times 10^{-5} \bar{P}_{t}^{2}+1.3382 \bar{P}_{t}
$$

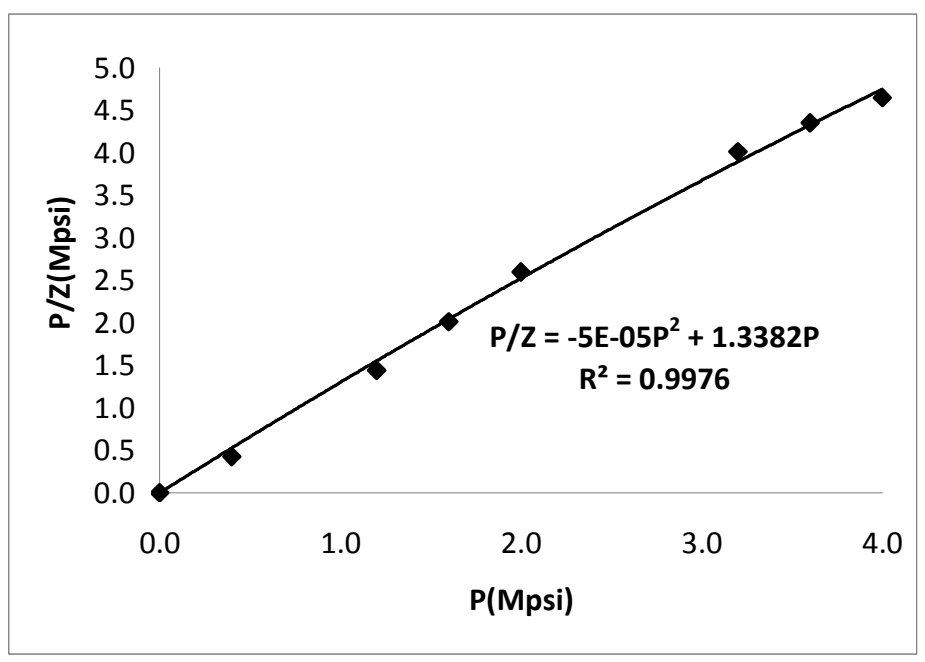

Figure 2-2 Correlation between $P / Z$ and $P$

The performance of each well is a function of average properties of the reservoir, the drainage volume, surface facilities and its own properties, and it is described by the productivity index equation: 


$$
q_{g s c, k}=J_{k}\left(\bar{P}^{2}-P_{w f, k}^{2}\right)^{n}
$$

Where

$$
\begin{array}{ll}
q_{g s c, k} & : \text { production from an individual well, Mscf/day } \\
J_{k} & : \text { Productivity index, Mscf/psi } \\
\bar{P} & : \text { Average reservoir pressure, } p s i \\
P_{w f, k} & : \text { Well flowing pressure, } p s i \\
n & : \text { Pressure exponent }
\end{array}
$$

The need for the correlation between the average pressure and the $\overline{\left(\frac{P}{Z}\right)}$ ratio comes from the productivity index equation, in which the average pressure is used to determine the production or injection rate:

$$
J_{k}=\frac{k h_{k}}{1422 \overline{\left(\mu_{g} Z\right)}\left(\frac{1}{2} \ln \left(\frac{4 A}{1.781 C_{A} r_{w, k}^{2}}\right)+s_{k}\right)}
$$

Also, we can verify that the $\mu \times Z$ product will remain relatively constant in the range below 2500 psi.

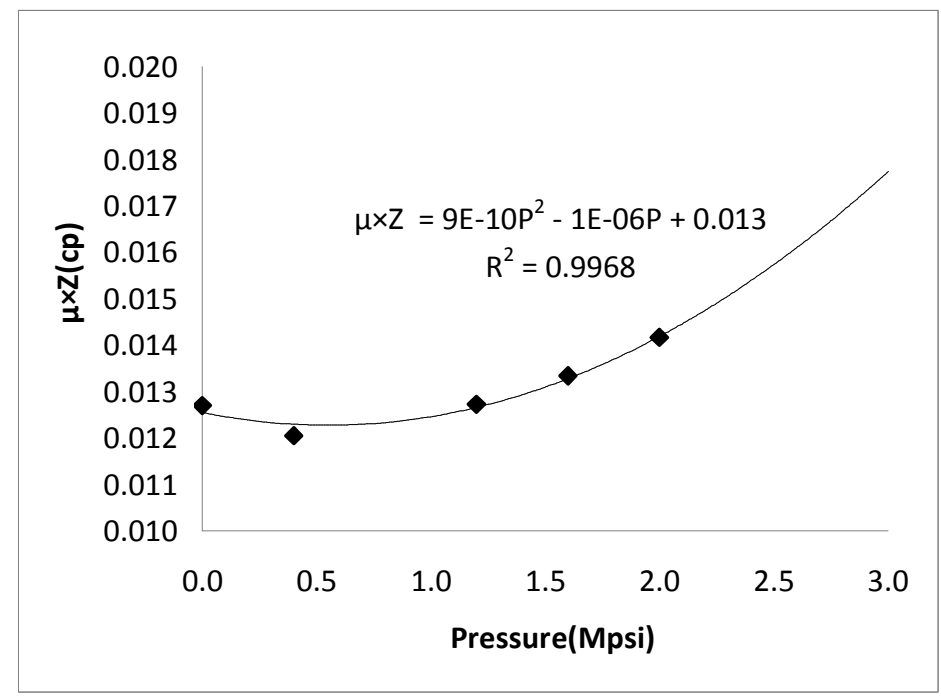

Figure 2-3 Correlation between $\mu \times Z$ and pressure

The choice on the production and injection rates is not completely free to change because of reservoir constraints. For example, there are constraints on the 
reservoir capacity, injection and production rates, productivity indices, bottomhole flowing pressure, injection pressure and average reservoir pressure. Table 2-3 summarizes the gas tank model equations. 
Table 2-3 Summary of Equations for dry gas tank model at low pressure

\begin{tabular}{|c|c|c|}
\hline $\begin{array}{l}\text { MBE } \\
\text { Continuous } \\
\text { form }\end{array}$ & $\frac{d \overline{\left(\frac{P}{Z}\right)}}{d t}=-\overline{\left(\frac{P}{Z}\right)} \frac{1}{G}\left[Q_{p r o, t o t}(t)-Q_{i n j, t o t}(t)\right]$ & $Q_{p r o, t o t}=\sum_{k=1}^{N_{w}} q_{g s c, k}=\sum_{k=1}^{N_{w}} J_{p r o, k}\left(\bar{P}^{2}-P_{w f, k}^{2}\right)^{n}$ \\
\hline $\begin{array}{l}\text { MBE } \\
\text { Discrete form }\end{array}$ & $\overline{\left(\frac{P}{Z}\right)_{t}}-\overline{\left(\frac{P}{Z}\right)_{t-1}}=-\overline{\left(\frac{P}{Z}\right)_{i}} \frac{1}{G}\left[Q_{p r o, t o t}(t)-Q_{i n j, t o t}(t)\right]$ & $Q_{i n j, t o t}=\sum_{k=1}^{m b} i_{g s c, k}=\sum_{k=1}^{m j} J_{i n j, k}\left(P_{i n j, k}^{2}-\bar{P}^{2}\right)^{2}$ \\
\hline $\begin{array}{l}\text { MBE } \\
\text { Cumulative } \\
\text { form, Monthly } \\
\text { basis }\end{array}$ & $\begin{array}{l}\overline{\left(\frac{P}{Z}\right)_{t}}=\overline{\left(\frac{P}{Z}\right)_{t-1}}-\overline{\left(\frac{P_{i}}{Z_{i}}\right)} \frac{1}{G}\left[\left(G_{p}(t)-G_{p}(t-1)\right)-(G\right. \\
G_{p r o}(t)=G_{p r o}(t-1)+30 \times \sum_{k=1}^{N_{p r o}} q_{k}(t), G_{i n j}(t)=G_{i n j}(\end{array}$ & $\begin{array}{l}\left.\left.t)-G_{I}(t-1)\right)\right] \\
-1)+30 \times \sum_{k=1}^{N_{p r o}} i_{k}(t)\end{array}$ \\
\hline $\begin{array}{l}\text { Productivity } \\
\text { index equation }\end{array}$ & $\begin{array}{l}q_{g s c, k}=J_{k}\left(\bar{P}^{2}-P_{w f, k}^{2}\right)^{n} \\
J_{k}=\frac{k h}{1422 \overline{\left(\mu_{g} Z\right)}\left(\frac{1}{2} \ln \left(\frac{4 A}{1.781 C_{A} r_{w, k}^{2}}\right)+s_{k}\right)}\end{array}$ & $\begin{array}{l}Q_{p r o, t o t}=\sum_{k=1}^{N_{w}} J_{p r o, k}\left(\bar{P}^{2}-P_{w f, k}^{2}\right)^{n} \\
Q_{i n j, t o t}=\sum_{k=1}^{N_{i n j}} J_{i n j, k}\left(P_{i n j, k}^{2}-\bar{P}^{2}\right)^{n}\end{array}$ \\
\hline PVT correlation & $\overline{\left(\frac{P}{Z}\right)_{t}}=-5 \times 10^{-5} \bar{P}_{t}^{2}+1.3382 \bar{P}_{t}$ & $\begin{array}{l}\text { Should be found based on the PVT data } \\
\text { Ideal gas assumption valid for P } \leq 300 \text { psi }\end{array}$ \\
\hline $\begin{array}{l}\text { Cumulative } \\
\text { rates }\end{array}$ & $\begin{array}{l}G_{p r o}(t)=G_{p r o}(t-1)+30 \times \sum_{k=1}^{N_{p r o}} q_{k}(t) \\
G_{i n j}(t)=G_{i n j}(t-1)+30 \times \sum_{k=1}^{N_{p r o}} i_{k}(t)\end{array}$ & $\begin{array}{l}\text { The relation depends on the choice of time } \\
\text { steps, here the time step is one month ( } 30 \\
\text { days) }\end{array}$ \\
\hline \multirow{5}{*}{$\begin{array}{l}\text { Implicit } \\
\text { equations }\end{array}$} & $\begin{array}{l}\overline{\left(\frac{P}{Z}\right)_{\text {Min }}} \leq \overline{\left(\frac{P}{Z}\right)_{t}} \leq \overline{\left(\frac{P}{Z}\right)_{M a x}} \\
\bar{P}_{\text {Min }} \leq \bar{P}_{t} \leq \bar{P}_{\text {Max }} \\
G_{\text {Base }} \leq G_{t} \leq G_{\text {Max }}\end{array}$ & $\begin{array}{l}\text { Minimum and Maximum capacities, they are all } \\
\text { the same }\end{array}$ \\
\hline & $P_{i n j, M i n} \leq P_{i n j, k}(t) \leq P_{i n j, \operatorname{Max}} \quad k=1,2, \ldots, N_{i n j}$ & Minimum/Maximum injection pressure \\
\hline & $q_{i n j, k}(t) \leq q_{i n j, \operatorname{Max}} \quad k=1,2, \ldots, N_{i n j}$ & Maximum injection rate \\
\hline & $P_{w f, \operatorname{Min}} \leq P_{w f, k}(t) \quad k=1,2, \ldots, N_{p r o}$ & Minimum Well Flowing pressure \\
\hline & $q_{p r o, k}(t) \leq q_{p r o, \operatorname{Max}} \quad k=1,2, \ldots, N_{p r o}$ & Maximum withdrawal rate \\
\hline
\end{tabular}




\subsection{Multilayer Gas Tank Model}

A multilayer reservoir consists of at least two layers with different reservoir properties. Layers can be in perfect communication, which means they have maximum interlayer crossflow. In this case there is a high vertical transmissibility between the layers, and hence the pressure difference between the layers is minimal. On the other hand the layers might be completely separated by an impermeable layer (seal or fault). In this case there is no communication between the layers and the compartments act independently. Between these two extremes, the reservoir performance might be affected by vertical transmissibility between the layers. Here we address the equations for a simple case of two layer reservoir with effective vertical transmissibility $\Gamma_{12}$. This model assumes:

- Single phase flow of dry gas

- Two layers, with vertical communication

- No communication along the wellbore

- Multiple wells drilled in each layer, each well is perforated in only one layer

- For each layer the respective productivity and injectivity indices are equal

- Gravity is neglected

- Layer one is the most permeable layer (PI1 >PI2)

We start with the MBE for the whole system, with the same assumptions as the previous section:

$$
\overline{\left(\frac{P}{Z}\right)}=\overline{\left(\frac{P_{i}}{Z_{i}}\right)}\left(1-\frac{G_{p}-G_{I}}{G}\right)
$$

Now, we write the MBE for each layer:

$$
\overline{\left(\frac{P}{Z}\right)_{t, 1}}=\overline{\left(\frac{P_{i}}{Z_{i}}\right)_{1}}\left(1-\frac{G_{p, 1}-G_{I, 1}-Q_{x f}}{G_{1}}\right)
$$




$$
\overline{\left(\frac{P}{Z}\right)_{t, 2}}=\overline{\left(\frac{\left.P_{i}\right)}{Z_{i}}\right)_{2}}\left(1-\frac{G_{p, 2}-G_{I, 2}+Q_{x f}}{G_{2}}\right)
$$

Where $Q_{x f}$ is the cumulative crossflow from layer two to layer one:

$$
Q_{x f}=\int_{0}^{t} q_{x f} d t^{\prime}
$$

$G_{I, i}$ and $G_{P, i}$ are cumulative production and injection from each layer:

$$
\begin{aligned}
G_{P, i} & =\int_{0}^{t} q_{i} d t^{\prime} \\
G_{I, i} & =\int_{0}^{t} I_{i} d t^{\prime}
\end{aligned}
$$

We discretize the MBE equations and subtract for each layer in the same way as for the single layer reservoir to get:

$$
\begin{aligned}
& \left.\overline{\left(\frac{P}{Z}\right)_{t, 1}}=\overline{\left(\frac{P}{Z}\right)_{t-1,1}}-\overline{\left(\frac{P}{Z}\right)}\right)_{1}\left(\frac{\left(G_{p, 1}(t)-G_{p, 1}(t-1)\right)-\left(G_{I, 1}(t)-G_{I, 1}(t-1)\right)-\left(Q_{x f}(t)-Q_{x f}(t-1)\right)}{G_{1}}\right) \\
& \overline{\left(\frac{P}{Z}\right)_{t, 2}}=\overline{\left(\frac{P}{Z}\right)_{t-1,2}}-\overline{\left(\frac{P_{i}}{Z}\right)_{i}}\left(\frac{\left(G_{p, 2}(t)-G_{p, 2}(t-1)\right)-\left(G_{I, 2}(t)-G_{I, 2}(t-1)\right)+\left(Q_{x f}(t)-Q_{x f}(t-1)\right)}{G_{2}}\right)
\end{aligned}
$$

Cumulative production, injection and crossflow can be related to their respective rates:

$$
\begin{array}{ll}
G_{I, i}(t)=G_{I, i}(t-1)+l_{p} \times \sum_{k=1}^{N_{i}} I_{k, i}(t) & i=1,2 \\
G_{p, i}(t)=G_{p, i}(t-1)+l_{p} \times \sum_{k=1}^{N_{i}} q_{k, i}(t) & i=1,2
\end{array}
$$




$$
Q_{x f}(t)=Q_{x f}(t-1)+l_{p} \times \sum_{k=1}^{N} q_{x f}(t) \quad N=\sum_{i=1}^{2} N_{i}
$$

The crossflow rate is proportional to the average pressure square difference between the two layers:

$$
q_{x f}(t)=\frac{0.111924 \Gamma_{v}\left(\bar{P}_{2}^{2}(t)-\bar{P}_{1}^{2}(t)\right)}{T h_{12}}
$$

Where $\Gamma_{v}$ is the vertical transmissibility coefficient between the layers, defined as

$$
\begin{aligned}
& \Gamma_{v}=\frac{\Gamma_{1} \Gamma_{2}\left(h_{1}+h_{2}\right)}{h_{1} \Gamma_{2}+h_{2} \Gamma_{1}} \\
& \Gamma_{1}=\left[\frac{k A}{Z \mu_{g}}\right]_{1} \\
& \Gamma_{2}=\left[\frac{k A}{Z \mu_{g}}\right]_{2}
\end{aligned}
$$

$A$ is the effective communication area between the two layers and $h_{12}$ is the average vertical distance between the centers of the two layers.

\subsection{Compartmental Reservoir Model}

A compartmental reservoir consists of two or more distinct regions that are allowed to communicate. Each compartment or "tank" is described by its own material balance, which is coupled to the material balance of the neighboring compartments through influx or efflux gas across the common boundaries. Payne (1996) and Hagoort and Hoogstra (1999) proposed two different robust and rigorous schemes for the numerical solution of the MBEs, of compartmented gas reservoirs (Ahmed and McKinney, 2005). Both schemes employ the following basic approach:

- Divide the reservoir into a number of compartments with each compartment containing one or more production wells that are proximate and that 
measure consistent reservoir pressures. The initial division should be made with as few tanks as possible with each compartment having different dimensions in terms of length $L$, width $W$, and height $h$.

- Each compartment must be characterized by a historical production and pressure decline data as a function of time.

- If the initial division is not capable of matching the observed pressure decline, additional compartments can be added either by subdividing the previously defined tanks or by adding tanks that do not contain drainage points, i.e., production wells.

A thin permeable layer with a transmissibility of $\Gamma_{12}$ is assumed to be separating the two compartments. Hagoort and Hoogstra expressed the instantaneous gas influx through the thin permeable layer by Darcy's equation as given by (in field units):

$Q_{12}=\frac{0.111924 \Gamma_{12}\left(P_{1}^{2}-P_{2}^{2}\right)}{T L}$

With

$$
\begin{aligned}
& \Gamma_{12}=\frac{\Gamma_{1} \Gamma_{2}\left(L_{1}+L_{2}\right)}{L_{1} \Gamma_{2}+L_{2} \Gamma_{1}} \\
& \Gamma_{1}=\left[\frac{k A}{Z \mu_{g}}\right]_{1} \\
& \Gamma_{2}=\left[\frac{k A}{Z \mu_{g}}\right]_{2}
\end{aligned}
$$

Where:

$$
\begin{aligned}
& Q_{12}=\text { influx gas rate, } s c f / \text { day } \\
& L=\text { distance between the centers of compartments, } f t \\
& A=\text { cross-sectional area, } f t^{2} \\
& \mu_{\mathrm{g}}=\text { gas viscosity, } c p \\
& Z=\text { gas deviation factor } \\
& K=\text { permeability, } m d \\
& P=\text { pressure, } p \text { sia } \\
& T=\text { temperature, } \circ R
\end{aligned}
$$


$L_{1}=$ length of compartment $1, f t$

$L_{2}=$ length of compartment $2, f t$

The subscripts 1 and 2 refer to compartments 1 and 2, respectively.

The material balance for the two reservoir compartments can be modified to include the gas influx from compartment 1 to compartment 2 as:

$$
\begin{aligned}
& \frac{P_{1}}{Z_{1}}=\frac{P_{i}}{Z_{i}}\left(1-\frac{G_{p 1}-G_{i 1}+G_{12}}{G_{1}}\right) \\
& \frac{P_{2}}{Z_{2}}=\frac{P_{i}}{Z_{i}}\left(1-\frac{G_{p 2}-G_{i 2}-G_{12}}{G_{2}}\right)
\end{aligned}
$$

Where

$$
\begin{aligned}
& Q_{12}=\text { influx gas rate, } s c f / d a y \\
& L=\text { distance between the centers of compartments, } f t \\
& A=\text { cross-sectional area, } f^{2}{ }^{2} \\
& \mu_{\mathrm{g}}=\text { gas viscosity, } c p \\
& Z=\text { gas deviation factor } \\
& k=\text { permeability }, m d \\
& P=\text { pressure, } p \text { sia } \\
& T=\text { temperature, } \circ R \\
& L_{1}=\text { length of compartment } 1, f t \\
& L_{2}=\text { length of compartment } 2, f t
\end{aligned}
$$

Considering the cumulative cross flow equation:

$$
\begin{aligned}
& G_{12}=\int Q_{12} d t \\
& Q_{12}=\frac{0.111924 \Gamma_{12}\left(P_{1}^{2}(t)-P_{2}^{2}(t)\right)}{T L}
\end{aligned}
$$

With

$$
\begin{aligned}
& Q_{12}=\text { influx gas rate, } s c f / d a y \\
& P_{1}, P_{2}=\text { Average pressures in compartment } 1 \text { and } 2, p s i
\end{aligned}
$$

The system has 7 unknowns with three equations; the cumulative production and injection for each layer is a single sign-free variable. Therefore the system has 2 degrees of freedom, which represent the net flowrate from each layer. Given the net rates, one can solve the MBE equations along with the crossflow equation for the 
two unknowns $P_{1}$ and $P_{2}$, the two MBE expressions can be arranged and equated to zero, to give

$$
\begin{aligned}
& F_{1}\left(P_{1}, P_{2}\right)=P_{1}-\frac{P_{i}}{Z_{i}} Z_{1}\left(1-\frac{G_{p 1}-G_{i 1}+G_{12}}{G_{1}}\right)=0 \\
& F_{2}\left(P_{1}, P_{2}\right)=P_{2}-\frac{P_{i}}{Z_{i}} Z_{2}\left(1-\frac{G_{p 2}-G_{i 2}-G_{12}}{G_{2}}\right)=0
\end{aligned}
$$

The general methodology of applying the method mainly involves the following specific steps:

1. Prepare the available gas properties data in tabulated and graphical forms that include $Z$ vs. $p$ and $\mu_{\mathrm{g}}$ vs. $p$.

2. Divide the reservoir into compartments and determine the dimensions of each compartments in terms of length (L), height (h), width (W) and crosssectional area $(A)$

3. For each compartment, determine the initial gas-inplace (G).For reasons of clarity, assume two gas compartments and calculate $G_{1}$ and $G_{2}$ from the following equations

$$
\begin{aligned}
& G_{1}=\frac{43560 A_{1} h_{1} \phi_{1}\left(1-S_{w i}\right)}{B_{g i}} \\
& G_{2}=\frac{43560 A_{2} h_{2} \phi_{2}\left(1-S_{w i}\right)}{B_{g i}}
\end{aligned}
$$

4. For each compartment, make a plot of $p / Z$ vs. $G_{p}$ that can be constructed by simply drawing a drawing a straight line between pi/Zi with initial gas-in-place in both compartments, i.e., $\mathrm{G}_{1}$ and $\mathrm{G}_{2}$.

5. Calculate the transmissibility by applying Equation...

6. Select a time step $\Delta t$ and determine the corresponding actual cumulative gas production $\mathrm{G}_{\mathrm{p} 1}$ and $\mathrm{G}_{\mathrm{p} 2}$. 
7. Calculate the gas crossflow rate $Q_{12}$ and cumulative gas influx $G_{12}$ by applying the following equations:

$$
\begin{aligned}
& Q_{12}=\frac{0.111924 \Gamma_{12}\left(P_{1}^{2}-P_{2}^{2}\right)}{T L} \\
& G_{12}=\int_{0}^{t} Q_{12} d t=\sum_{0}^{t} \Delta Q_{12} \Delta t
\end{aligned}
$$

8. Start the iterative solution by assuming initial estimates of the pressure for compartments 1 and 2 (i.e., $P_{1}^{k}$ and $P_{2}^{k}$ ). Using the Newton- Raphson iterative scheme, calculate new improved values of the pressure $P_{1}^{k+1}$ and $P_{2}^{k+1}$ solving the following linear equations as expressed in a matrix form:

$$
\left[\begin{array}{c}
P_{1}^{k+1} \\
P_{2}^{k+1}
\end{array}\right]=\left[\begin{array}{c}
P_{1}^{k} \\
P_{2}^{k}
\end{array}\right]-\left[\begin{array}{cc}
\frac{\delta F_{1}\left(P_{1}^{k}, P_{2}^{k}\right)}{\delta P_{1}} & \frac{\delta F_{1}\left(P_{1}^{k}, P_{2}^{k}\right)}{\delta P_{2}} \\
\frac{\delta F_{2}\left(P_{1}^{k}, P_{2}^{k}\right)}{\delta P_{1}} & \frac{\delta F_{2}\left(P_{1}^{k}, P_{2}^{k}\right)}{\delta P_{2}}
\end{array}\right]^{-1} \times\left[\begin{array}{c}
F_{1}\left(P_{1}^{k}, P_{2}^{k}\right) \\
F_{2}\left(P_{1}^{k}, P_{2}^{k}\right)
\end{array}\right]
$$

9. The superscript 1 denotes the inverse of the matrix. The partial derivatives in the above system of equations can be expressed in analytical form by differentiating Equations for $F_{1}$ and $F_{2}$ with respect to $p 1$ and $p 2$. During an iterative cycle, the derivatives are evaluated at the updated new pressures, i.e. $P_{1}^{k}$ and $P_{2}^{k}$. The iteration is stopped when $\left|P_{1}^{k+1}-P_{1}^{k}\right|$ and $\left|P_{2}^{k+1}-P_{2}^{k}\right|$ are less than a certain pressure tolerance, i.e., 5-10 psi.

10. Generate the pressure profile as a function of time for each compartment by repeating steps 2 and 3.

11. Repeat steps 6 through 10 to produce a pressure decline profile for each compartment that can be compared with the actual pressure profile for each compartment or that from step 4. 
12. Compare the calculated pressure profiles with those of the observed pressures. If a match has not been achieved, adjust the size and number of compartments (i.e., initial gas in- place) and repeat steps 2 through 10.

\subsection{Multi Layer - Multi Compartment Reservoir}

The multi layer-multi compartment reservoir is the combination of the two models, multi layer model and multi compartment model. The only complexity of this problem is the indexing and mutual transmissibility factors between layercompartments.

Table 2-4 summarizes the equations for the multi layer model. Note that the multi compartment model has the same structure, except for the notations used to represent for compartments and crossflows. 
Table 2-4 Summary of equations for multilayer reservoir

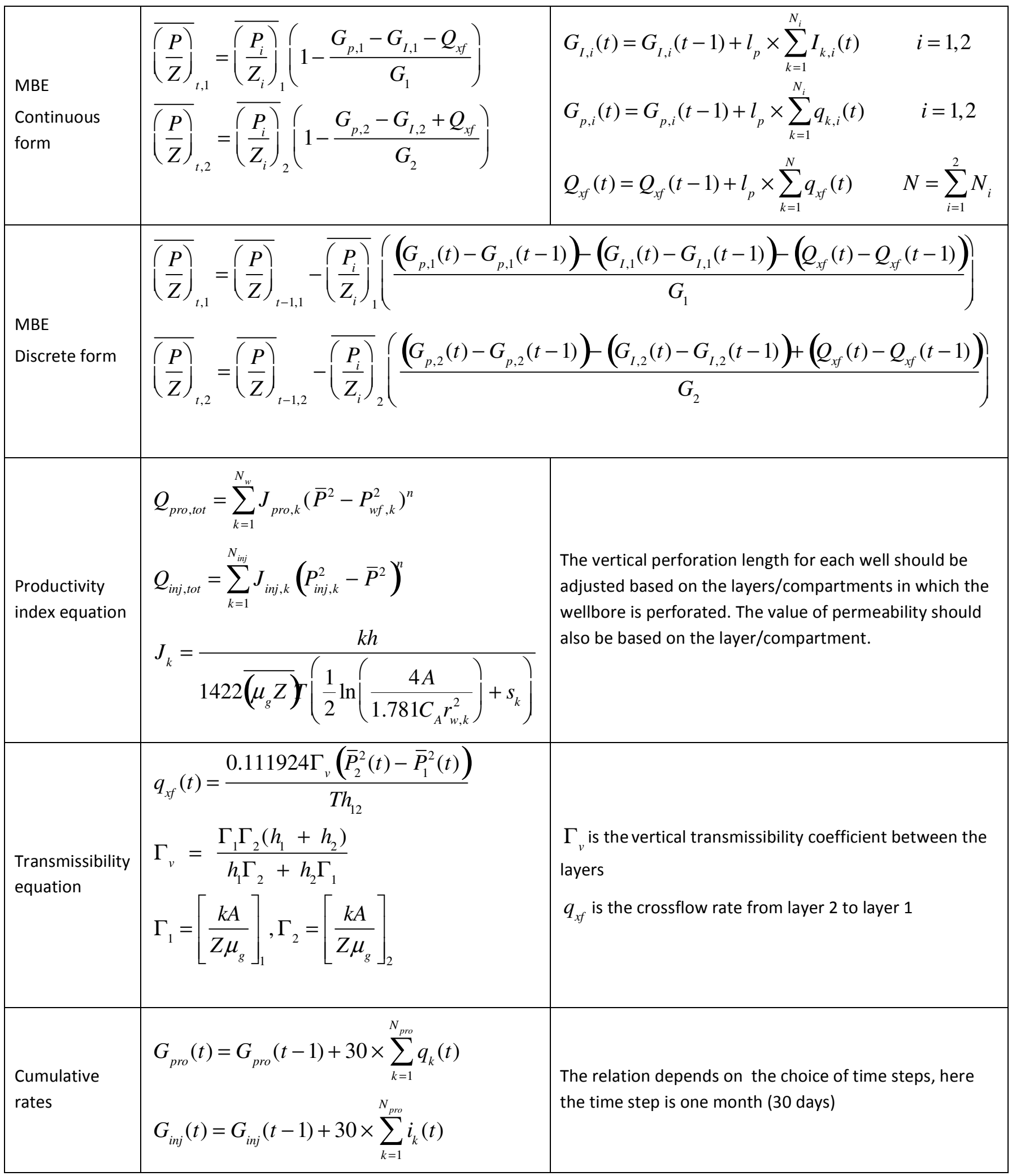




\section{Gas Storage Facility Model}

The motivation for gas storage comes from the fact that the demand for gas is cyclical. During the hot months, it goes down and during the cold months it goes up. Gas storage can be viewed as an inventory where gas can be injected and stored, and then be produced when needed. The gas storage serves as a buffer: it is used to balance the market supply and demand. Gas storage is also used to respond to unexpected demand, for example in extreme weather conditions.

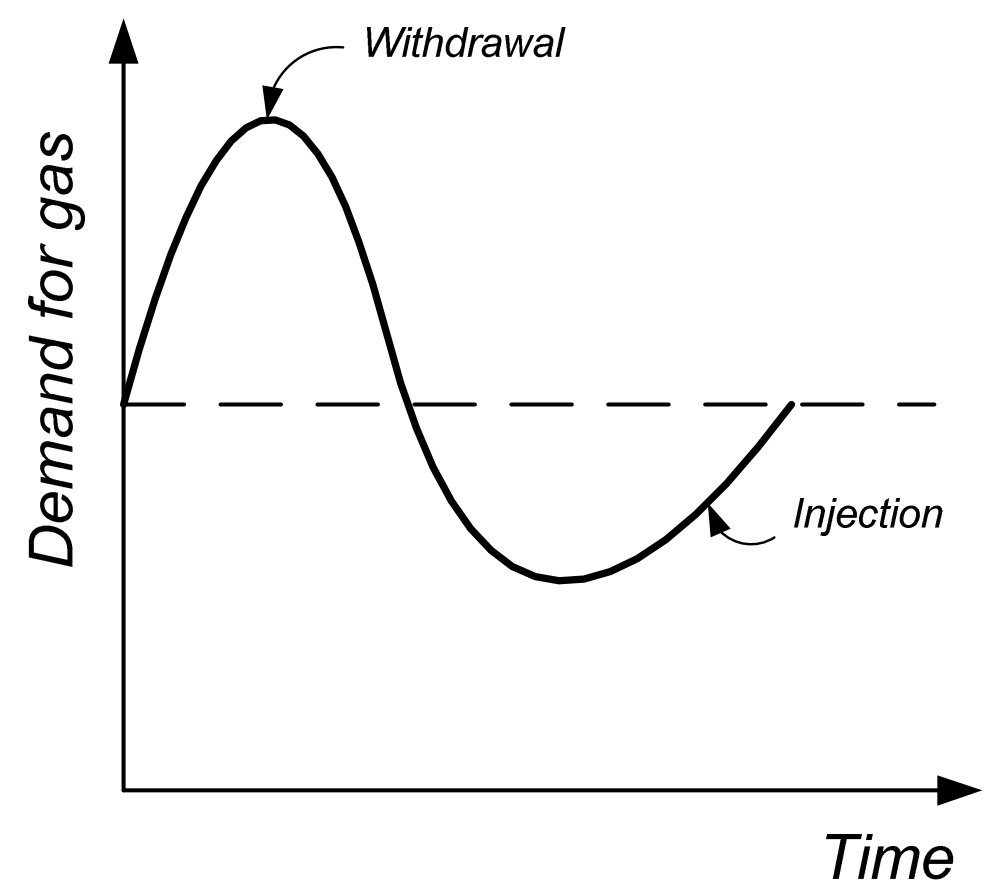

Figure 3-1 General annual trend for market demand 
Designing a gas storage system is a complicated process: the main design decisions include:

- Reservoir size

- Number of wells

- Compression horsepower

- Dehydration capacity

- Base gas volume

- Withdrawal/Injection rates

These decisions are based the estimates and predictions for the requirements and the expected performance of the reservoir and facilities. These estimates mainly include:

- Reservoir properties

- Demand

- Injection and withdrawal performance

- CAPEX

- OPEX

- Regulations

One of the main challenges of designing a storage system is the uncertainty in the estimates. For a storage system, the questions of when and how much to produce or inject are commonly posed since they are directly related to the financial benefits gained from the storage activities. Integral to these questions is the role of uncertainty in many of the inputs to the analysis (e.g. market demand). A sound design configuration and production-injection schedule accounts for the basic relations between the design variables and estimates, as well as the uncertainties. As a result, design and development of gas storage systems under uncertainty is a 
complicated and interesting problem. The purpose of the current chapter is to provide a model to answer this problem.

In this chapter, we describe the major features of a gas storage system. After a brief review of the related works on gas storage facility design problems, we present our version of the problem. Based on the reservoir tank model we propose a mathematical formulation for the gas storage. Finally, we discuss the significant input parameters of the proposed model.

\subsection{Literature Review on Gas Storage Facility Design and Planning}

Carlos and Chu (1973) analyzed the effect of uncertainty in weather conditions on the demand cycle of gas storage; they developed a computer code based on the single block tank model assumptions and a periodic demand curve with normally distributed peak and used Monte Carlo simulation for different combinations of compression horsepower and wells to find the optimal solution. Their work assumed a constant value for the base gas volume.

Coats (1969) performed a two dimensional numerical calculation for the semi steady-state pressure distribution and individual well deliverabilities in a gas field producing under a specified total rate schedule. It accounted for reservoir heterogeneity, irregular well spacing and drainage areas, unequal well rates, and well interference effects. He used the model to estimate field performance for any given combination of producing well locations and also to determine an optimal order of drilling for a given set of admissible well sites.

Wattenbarger (1969) formulated a linear programming problem to find an optimal withdrawal schedule that maximizes total gas withdrawals during the producing season, subject to constraints of fixed demand schedule and minimum wellbore pressure. He used the concepts of real gas pseudo pressure and the superposition of finite-difference solutions through the application of transient influence matrices to linearize and solve the problem. 
Van Horn and Wienecke (1970) solved the gas-storage-design optimization problem with a Fibonacci Search algorithm. They expressed the investment requirement for a storage field in terms of four variables: cushion gas, number of wells, purification equipment, and compressor horsepower. They chose the combination of these four variables that minimized investment cost as the optimum design criteria. The authors used an empirical backpressure equation, combined with a simplified gas material-balance equation, as the reservoir model.

McVay and Spivey (2001) provided a comprehensive literature review on different gas storage problems and the techniques applied to solve them. They briefly discussed the strengths and weaknesses of the assumptions, solution techniques and results presented in the previous works. They pointed out that all previous works have either used reservoir simulators to account for complex heterogeneity effects of reservoir on the optimal injection-withdrawal policy, or used single gridblock tank models to analyze the effects of costs and market on the optimal combination of wells horse power and cushion gas. They used reservoir simulation to examine a practical range of compression horsepower, number of wells and cushion gas and determined the combination that minimizes the objective function, defined as the initial development cost. They noted that the procedure proposed may not find the absolute minimum cost; it will only find the lowest cost of the combinations tested; they implied that the actual gas storage problem is actually a constrained optimization problem and more sophisticated methods may be applied to find the absolute minimum.

Huppler (1974) specified a peak delivery and production schedule for a homogenous gas reservoir and evaluated different combinations of wells and compression using a nonlinear dynamic programming model. The van EverdingenHurst unsteady state model was used to account for the water influx and the effect of production policy on the recovery efficiency was evaluated.

Hower et al. (1993) used a 3D reservoir simulator (ECLIPSE) to address the performance improvement and water encroachment issues of aquifer gas storage 
reservoirs. They used a model consisting of $6 * 6$ blocks with 6 layers is developed in eclipse. They used the real gas storage field data and calibrated the model using the past performance of reservoir (history matching). Using the results, they addressed the issues of using peripheral wells for withdrawal against uniform field wide depletion, effects of peak day versus base loading on water encroachment, the effects of shut-in time between injection and withdrawal and the effects of increased injected volumes. Khodri et al. (1997) also used the same methodology to model gas storage reservoirs and address the problems of improving the peak flow rate, maximizing the performance and deliverability and decision on drilling new wells.

Brown et al. (1999) used a homogeneous volumetric tank model to solve various storage problems, such as determining the minimum withdrawal time (or the peak withdrawal rate), determining the minimum injection time (peak injection rate) and finding the optimal number of storage cycles. They remarked the limitations of the reservoir model used; however, they noted that using inexpensive tools and data are sufficient for storage operations.

Kuncir et al. (2003) used tank model with single phase gas flow, coupled with a surface flow system to evaluate the development and expansion options for a gas storage reservoir. They developed an optimization code to evaluate different horsepower and well count configurations to meet a demand schedule. The problem has not accounted for the optimal amount of the base gas. Naturally, the estimated value of demand can be considered as the solution for the working gas. They also estimated the aquifer parameters, represented by the Fetkovitch model, as well as the parameters for a depleted oil reservoir.

Johnson et al. (2000) remarked that the computation time is the main barrier to the integration of reservoir simulators and optimization techniques. They pointed out that generally in E\&P development projects the emphasis is on selection of small, carefully selected scenarios. 
The design and development of underground gas storage systems requires the selection of optimal values for several decision variables. There are also market and subsurface uncertainties in any oil and gas project that affect the ultimate value derived from the project. So, there is a need to first integrate the reservoir model, facilities model and economic model into an optimization platform, and second to incorporate the uncertainties and investigate their on output decision variables. The literature review on the related works previously performed in this area has shown that these analytical tools and techniques are not integrated to consider all these factors together, namely, subsurface behavior, market and economic equations and the related uncertainties.

\subsection{Selected Approach to Problem Definition}

There are two conventional approaches to specifying a gas storage facility design problem: asset-based and requirement-based.

In the asset based approach, it is assumed that the storage medium is known, and the optimization of a performance attribute is performed under this constraint. The performance attribute is typically defined as the net present value or the total volume of working gas or the peak deliverability rate. In the requirement based approach, the minimum requirement for a performance attribute is selected and the system is optimized to satisfy the requirements in the most efficient manner. Here, we refer to the minimum requirements as functionality requirements. Functionality requirements can be defined as the minimum peak rate deliverability and peak rate duration, total working gas volume or minimum production and injection rates. The measure of efficiency can be defined as the total investment cost, average investment cost per working gas volume, or the annual investment and operation \& maintenance cost. Obviously, the objective of this approach is to cover the requirements with the minimum cost.

In this thesis, the analysis is performed using the requirement based approach. However, this choice is not intended to imply that the requirement based approach 
is superior to the asset based approach. Obviously, each of these approaches has its own costs and benefits.

\subsection{Basic Definitions for Gas Storage Facility}

There are several volumetric measures used to quantify the fundamental characteristics of an underground storage facility and the gas contained within it. For some of these measures, it is important to distinguish between the characteristic of a facility such as its capacity, and the characteristic of the gas within the facility such as the actual inventory level. These measures naturally impose some constraints on the rates, which are presented below. The measures are as follows:

Total gas storage capacity is the maximum volume of gas that can be stored in an underground storage facility in accordance with its design, which comprises the physical characteristics of the reservoir, installed equipment, and operating procedures particular to the site.

Total gas in storage is the volume of storage in the underground facility at a particular time.

Base gas (or cushion gas) is the volume of gas intended as permanent inventory in a storage reservoir to maintain adequate pressure and deliverability rates throughout the withdrawal season.

Working gas capacity refers to total gas storage capacity minus base gas.

Working gas is the volume of gas in the reservoir above the level of base gas. Working gas is available to the marketplace.

Deliverability is most often expressed as a measure of the amount of gas that can be delivered (withdrawn) from a storage facility on a daily basis. Also referred to as the deliverability rate, withdrawal rate, or withdrawal capacity, deliverability is usually expressed in terms of millions of cubic feet per day (MMcf/day). Occasionally, deliverability is expressed in terms of equivalent heat content of the gas withdrawn from the facility, most often in dekatherms per day (a therm is 
$100,000 \mathrm{Btu}$, which is roughly equivalent to 100 cubic feet of natural gas; a dekatherm is the equivalent of about one thousand cubic feet (Mcf)). The deliverability of a given storage facility is variable, and depends on factors such as the amount of gas in the reservoir at any particular time, the reservoir pressure, compression capacity, the configuration and capacities of surface facilities, and other factors. In general, a facility's deliverability rate varies directly with the total amount of gas in the reservoir: it is at its highest when the reservoir is full and declines as working gas is withdrawn.

Injection capacity (or rate) is the complement of the deliverability or withdrawal rate-it is the amount of gas that can be injected into a storage facility on a daily basis. As with deliverability, injection capacity is usually expressed in MMcf/day, although dekatherms/day is also used. The injection capacity of a storage facility is also variable, and is dependent on factors comparable to those that determine deliverability. By contrast, the injection rate varies inversely with the total amount of gas in storage: it is at its lowest when the reservoir is full and increases as working gas is withdrawn.

None of these measures are fixed or absolute. The rates of injection and withdrawal change as the level of gas varies within the facility. Additionally, in practice a storage facility may be able to exceed certificated total capacity in some circumstances by exceeding certain operational parameters. But the facility's total capacity can also vary, temporarily or permanently, as its defining parameters vary. Further, the measures of base gas, working gas, and working gas capacity can also change from time to time. This occurs, for example, when a storage operator reclassifies one category of gas to the other, often as a result of new wells, equipment, or operating practices (such a change generally requires approval by the appropriate regulatory authority). Also, storage facilities can withdraw base gas for supply to market during times of particularly heavy demand, although by definition, this gas is not intended for that use. 


\subsection{Model Description}

Selecting the appropriate model to analyze a dynamic system is a crucial engineering task and it requires knowledge and experience about the real system. Also, the level of detail included in the model depends on the decision(s) for which the model is being used, availability of information, significance of the details, time limitations and the computational effort required. The model should be sufficiently detailed to describe the dynamics of the system. Making assumptions that are too simplistic may cause a failure to model the salient features of the real system, while too much detail may turn the whole system away from its ultimate goal (e.g. decision support) and delay the results. So, for our model definition, we should reach a compromise between these two extremes. Considering this fact, we follow up to the model definition and formulation.

The major design variables of the gas storage system are:

- Reservoir size

- Number of wells

- Compression horsepower

- Dehydration capacity

- Base gas volume

- Withdrawal/Injection rates

For the current formulation of gas storage design problem and the proposed case studies, we assume that we already selected the reservoir and now we are interested in finding the best configuration of wells, compression and base gas to satisfy the functional requirements. The decision variables considered in the current model include the number of wells, compression horsepower, base gas volume, and withdrawal/injection rates.

In this section, we discuss the model assumptions and present the formulation of the requirement based problem. 


\subsubsection{Assumptions}

The major assumptions of the gas storage system are:

1. The reservoir is a tank, i.e. it follows all the tank model assumptions and equations for gas reservoirs. These assumptions and equations are fully described in Chapter 2.

2. The maximum reservoir pressure is equal to the initial reservoir pressure and the reservoir temperature is constant.

3. The whole storage cycle is one year.

4. There is one withdrawal season and one injection season. The duration of withdrawal season is five months and the duration of injection season is seven months. There is no overlap between the two seasons; however it's allowed to withdraw during the injection season and vice versa.

5. During the withdrawal season the reservoir is required to produce a minimum volume of gas, i.e. a monthly rate. During the injection season, the reservoir should store the required volumes.

6. The volume of gas remaining in the reservoir at the end of withdrawal season is the base gas volume. The volume of gas to be withdrawn during the whole withdrawal season is the working gas volume.

7. All wells are used both for withdrawal and injection. The individual withdrawal or injection rates can change, and they are determined by the optimization. However, the flow rates cannot exceed a maximum, to account for the wellbore/pipeline flow capacity and to avoid sand production.

8. Compression is used to adjust the bottomhole pressures. For production, the bottomhole pressure can be reduced to make a bigger pressure gradient and hence increase the flow rate. For injection, the bottomhole pressure can be increased to increase the injection rate. However, a minimum bottomhole pressure for production is assumed to guarantee that the gas can reach the 
surface; this is because the wellbore pressure drop and vertical lift performance. Similarly, the injection pressure is assumed to have a maximum based on the compression equipment.

9. The pipeline pressure is fixed throughout the whole cycle. The produced or injected gas has the same pressure as the pipeline when it flows in or out of the storage system.

10. The decision variables are:
a. The number of wells
b. Compression horsepower
c. Base gas volume
d. The withdrawal and injection rates

11. The objective is to minimize the investment cost for the storage facility, so that the storage system is able to cover the required withdrawal and injection rates. The investment cost includes the well cost, compression facility cost and the base gas cost.

\subsubsection{Problem Formulation}

The gas storage model is formulated as a mathematical programming model, which consists of an objective function and a set of constraints. As was discussed above, we selected to focus on the requirement based approach, and hence in this section we present the mathematical formulation of the requirement based design problem. The asset based problem can be presented by modifying the requirement based problem, which is presented in chapter 6 .

\subsubsection{Objective Function}

Several measures can be defined for the objective function. For the requirement based approach, the goal is to satisfy a functionality requirement with the minimum investment cost. Specifically, the measure of investment cost is capital 
investment cost per working gas volume. This measure is also appropriate for analysis of uncertainty in subsurface reservoir properties, supply and demand, peak rates and peak rate durations.

The objective is:

Minimize: Total cost $=$

Total discounted purchase cost for working gas+

Total purchase cost for base gas+

Total cost of wells+

Total compression cost+

Total discounted cost of $O \& M$

The measure of performance, which is used to compare different configurations is defined as the total investment cost per working gas volume, and

Total investment cost $=$

Total purchase cost for base gas+

Total cost of wells+

Total compression cost+

Note that the gas purchase price and operation \& maintenance costs are included to prevent unnecessary injection and withdrawal. However they are not included in the measure of performance which is used to compare different configurations.

\subsubsection{Reservoir and Well Equations}

Reservoir and well constraints describe the reservoir and well behavior. As we discussed in chapter 2, we selected the tank model to describe the reservoir behavior. Table 3-1 summarizes the reservoir properties selected as a case study. 
Table 3-1 Basic Reservoir Properties

\begin{tabular}{|l|l|}
\hline \multicolumn{1}{|c|}{ Reservoir Property } & \multicolumn{1}{c|}{ Descriptor or Value } \\
\hline Original Contents & Oil and Gas \\
\hline Type of Geological Trap & Stratigraphic \\
\hline Lithology & Sandstone \\
\hline Areal Extent of Reservoir & 7,000 acres \\
\hline Gross Thickness & 22 feet \\
\hline Depth to Formation & 6,200 feet \\
\hline Discovery Pressure & 2,400 psi \\
\hline Permeability & $50 \mathrm{md}$ \\
\hline Temperature & $600^{\circ} \mathrm{R}$ \\
\hline
\end{tabular}

\subsubsection{Compression and Associated Horsepower Requirements}

The compression system is used to increase the produced gas pressure to pipeline pressure for delivery, and to inject gas into the reservoir. The theoretical basis for gas compression and horsepower calculations are widely discussed in facility design literature. Here, we present the summary of equations used to correlate the surface pressure, bottomhole pressure, flow rates and compression horsepower. We assume constant pipeline pressure equal to $1000 \mathrm{psi}$, an isentropic exponent equal to 1.228 , and compressor efficiency of 0.82 .

$$
\begin{aligned}
& H P=\operatorname{Max}_{i}\left\{H P_{p r o, i}+H P_{i n j, i}\right\} \\
& H P_{p r o, i}=0.243446 \times Q_{p r o, i} \times\left\{\left(\frac{P_{p i p e l i n e}}{P_{w h, i}}\right)^{0.2236}-1\right\} \\
& H P_{i n j, i}=0.243446 \times Q_{i n j, i} \times\left\{\left(\frac{P_{i n j, i}}{P_{p i p e l i n e}}\right)^{0.2236}-1\right\}
\end{aligned}
$$


$H P_{p r o, i}[=\mathrm{HP}]$ is the nominal compression horsepower required for production and delivery of gas at pipeline pressure in period i. $Q_{p r o, i}$ [=MScf] and $Q_{i n j, i}$ [=MScf] are total production and injection rates in period $i . P_{\text {pipeline }}[=\mathrm{psi}]$ is the pipeline pressure and $P_{w h, i}\left[=\right.$ psi] and $P_{i n j, i}[=$ psi] are the wellhead pressure (for producers) and injection pressure (for injectors), respectively. The total theoretical HP required is the maximum of periodic HPs over the whole storage cycle.

\subsubsection{Functionality Requirements}

The functionality requirements are defined as the minimum withdrawal and injection rates in each month. Depending on the requirements, the model can decide on the withdrawal and injection rates for individual wells. The net injection/ production rate of the system for each month should be at least equal to the required rate to satisfy this constraint.

Chapter 5 discusses the dynamics of the presented equations. The corresponding code in GAMS is presented in Appendix $A$. 


\section{Uncertainty Analysis Workflow}

In this chapter, we define the uncertainty analysis workflow and its constituent parts. First, a step by step description of the workflow is provided. Second, a description of the integrated optimization model is given. This description includes an overview, definition of a reservoir model, the facility choices, and the economic optimization criterion. Similar versions of this workflow have been utilized to assess facility optimization and value of information problems for oil and gas production assets (see Jablonowski, Wiboonkij-Arphakul, and Neuhold (2008), Purwar (2008), and Hosgor (2009)). 
The uncertainty analysis workflow consists of 6 major steps as depicted in Figure 4-1. A detailed description of each step is provided below.

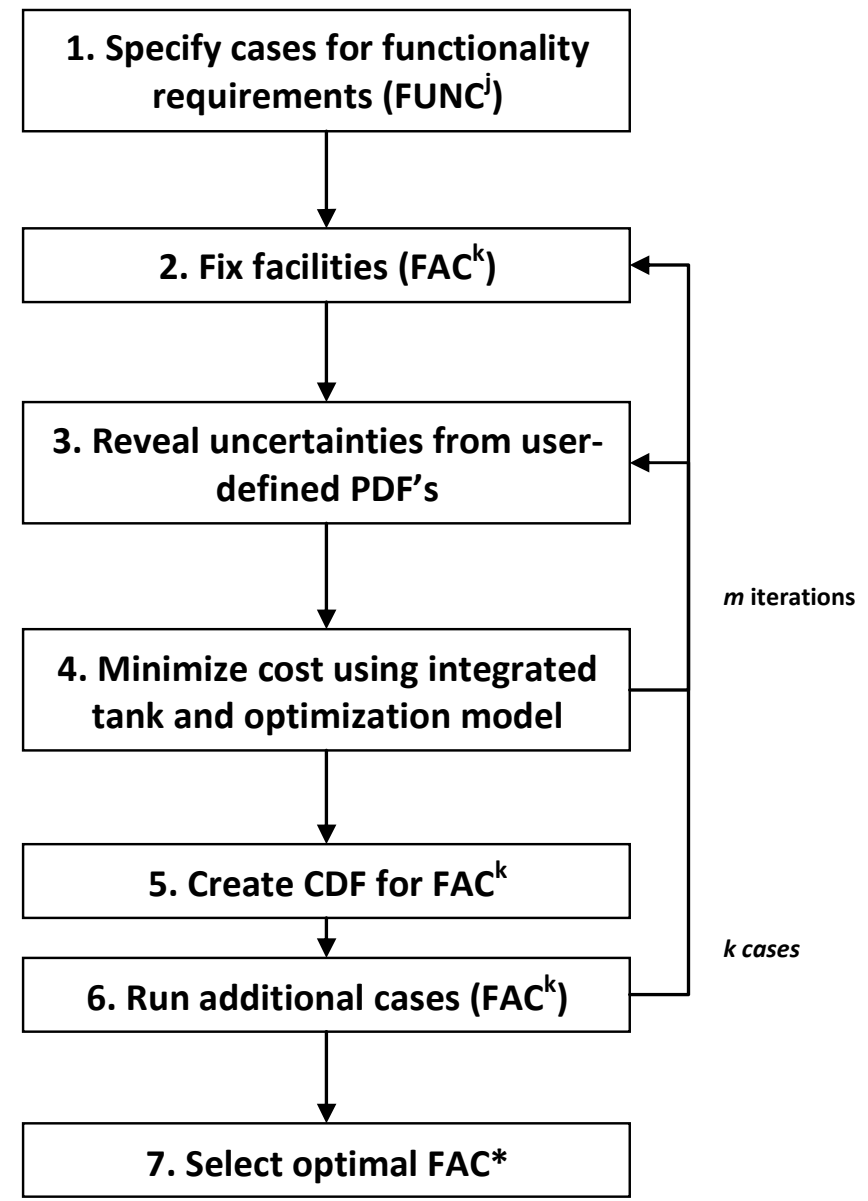

Figure 4-1 Uncertainty Analysis Workflow

\subsection{Step 1: Specify the Functionality Requirements.}

There are at least two major paths to take in gas storage facility design. One approach is to assume that a reservoir has been identified and then design a facility to maximize a particular performance attribute such as working gas or maximum production rate. A second approach is to define functionality requirements (e.g. minimum required production rate of $\mathrm{q}$ mmcf/day in month i) and solve for the costminimizing facility configuration that satisfies the requirement. ${ }^{4}$ These are related,

\footnotetext{
${ }^{4}$ When the reservoir is not already identified, this approach indicates what size of reservoir should be acquired.
} 
but fundamentally different problems. Procedures for both of these approaches are proposed and demonstrated in McVay and Spivey (2001). In this research, we investigate the second approach. That is, given a functionality requirement, we solve for the cost-minimizing configuration of wells and compression. It is assumed that the desired functionality derives from the operator's commercial analysis of supply and demand, current and potential competitors, and coordination with existing assets in the portfolio. ${ }^{5}$ Like McVay and Spivey (2001), we assume the storage cycle of injection and production requirements are specified as variable net volumes over time, ${ }^{6}$ but in contrast, we do not specify (constrain) the working gas volume.

The functionality requirements for production and injection rates can be specified deterministically or probabilistically. In deterministic cases, the operator defines the minimum net production rate requirements for months in the production interval, and the minimum net injection rate requirements for months in the injection interval. When specified probabilistically, the production rate and injection rate requirements in each month are specified as probability density functions (PDFs). This structure may model actual demands more realistically, and it also facilitates a sensitivity analysis on the impact of uncertainty in functionality requirements on optimal facility design. In this research, we specify rate requirements probabilistically. We specify November as Month 1. Months 1 to 5 (November to March) represent the production interval and months 6 to 12 (April to October) represent the injection interval.

\footnotetext{
${ }^{5}$ In practice, the specification of functionality requirements and facility optimization may be iterative and not sequential.

${ }^{6}$ For a one-cycle facility, the operating year consists of an injection interval and a production interval. Here, we specify net injection or production in each month. However, it should be recognized that injection can and does occur in months during the production period (when demand is low). Similarly during the injection interval, production may occur (to meet spikes in demand). There are also days during the storage cycle when the reservoir may be shut-in for various reasons such as reservoir monitoring, compressor or dehydrator maintenance, etc. Future research may relax this constraint so that intraperiod injection and production can be studied.
} 


\subsection{Step 2: Fix the Facility Configuration}

The uncertainties in estimates are assumed to be revealed after the facility is put in use and additional information is acquired (e.g. actual reservoir performance). Therefore, the problem is necessarily one of constrained optimization, and the facility constraints must be defined prior to revelation of the uncertain variables and subsequent optimization. In this study, we examine two decision variables: the number of wells and the compression horsepower. Additional decision variables can be investigated (e.g. dehydration); we limit the analysis here to facilitate the graphical analysis and discussion.

\subsection{Step 3: Reveal Uncertain Variables}

We make the simplifying assumption that the uncertain variables are revealed at the beginning of the optimization. This assumption introduces some anticipatory error into the computations because in practice, revelations are often imperfect and may occur over many months or years (each variable is different in this regard). This approach will yield the best solution (e.g. least cost) which in practice would not always be attained. However, other alternatives for modeling the uncertainty revelation are no less problematic. Deviating from a simplified approach would require assumptions about the timing and accuracy of new information, and the means and speed by which it is incorporated into optimization decisions. If the alternate assumptions are inaccurate, some error remains. From a practical perspective, the error from our simplifying assumption is unlikely to have a material impact on achieving the overall purpose of the analysis-uncertainty analysis for screening and concept selection.

\subsection{Step 4: Minimize the Total Cost for the Facility}

After the well count and horsepower are fixed in Step 2, and the uncertain variables are revealed in Step 3, the integrated reservoir-facility-economic optimization model is used to compute the least cost production and injection profile that satisfies the functionality constraint. This model simultaneously accounts 
for reservoir behavior, facility design, and economic objectives. The attractiveness of this approach is that the model is fast-solving and can be used in an iterative fashion. That is, the model is structured so that the uncertain variables can be resampled in Step 3 and the model re-solved. The resulting output is exceptionally rich in describing the potential outcomes for the specific facility configuration specified in Step 2.

\subsection{Step 5: Create the Probability Density Function for the Total Cost}

The output from Step 4 can be evaluated using various metrics. Here, we assess capital cost per volume of working gas (\$/BCF). The results can be reported as a PDF or a cumulative density function (CDF) or both. In this way, engineers and decision-makers can observe the shape and parameters of the simulated metric, and this information can be used to compare and contrast the competing facility configurations.

\subsection{Step 6: Obtain the Optimal Configuration}

In this step, other combinations of wells and horsepower are specified and additional results are generated. The objective is to continue running different configurations until an apparent global minimum is observed. ${ }^{7}$

\footnotetext{
${ }^{7}$ A method for specifying the facility cases to be run is to first specify the model with the number of wells and horsepower as endogenous variables. The results provide reasonable expectations for the optimal solutions in the constrained case.
} 


\section{Case Studies}

In this chapter, the proposed workflow and integrated model are used to investigate several stylized problems in gas storage facility design under uncertainty. The problems were selected to demonstrate the variety of uncertainties that can be evaluated using the workflow and model. In practice, there is uncertainty in demand, reservoir properties, capital costs, and other variables. Each variable is likely to have a unique impact on the optimal facility configuration. Also, the joint impact of multiple uncertainties is of interest.

As discussed in Chapter 3, there are two conventional approaches for gas storage design: asset based and requirement based. Each approach has its own applications. For the asset based approach, we defined one problem to illustrate the general features, namely, deterministic analysis for the net present value.

This research is mainly focused on the requirement based approach, and the, we defined four problems as follows:

1. Problem A: Deterministic Analysis for Functionality Requirements

2. Problem B: Uncertainty Analysis for Functionality Requirements

3. Problem C: Uncertainty Analysis for Reservoir Properties

4. Problem D: Joint Uncertainty Analysis

The choice of problems is mostly based on the natural trend one would follow to perform a thorough uncertainty analysis for a gas storage development optimization problem. Each problem investigates a specific type of uncertainty that may have an impact on the decision variables. 


\subsection{Problem Initialization}

\subsubsection{Reservoir Parameters}

Table 5-1 summarizes the reservoir properties used for the analysis. The choice of the reservoir properties is based on a real case. More detail on the reservoir and model properties is available in Appendix B.

Table 5-1 Reservoir Parameters

\begin{tabular}{|l|l|l|}
\hline Parameter & \multicolumn{1}{|c|}{ Description } & \multicolumn{1}{c|}{ Value } \\
\hline$P_{\max }$ & Maximum reservoir pressure & $2600 \mathrm{psi}$ \\
\hline$P_{\min }$ & Minimum reservoir pressure & $400 \mathrm{psi}$ \\
\hline$G_{B}$ & Base gas volume & $12.48 \mathrm{bcf}$ \\
\hline$G_{\max }$ & Total storage capacity (at $\left.P_{\max }\right)$ & $140.6 \mathrm{bcf}$ \\
\hline$A$ & Total area of the reservoir & $6000 \mathrm{acres}$ \\
\hline$\Phi$ & porosity & 0.15 \\
\hline$S_{w i}$ & Irreducible Water Saturation & 0.4 \\
\hline$T$ & Temperature & $600^{\circ} \mathrm{R}$ \\
\hline$h$ & Reservoir thickness & $20 \mathrm{ft}$ \\
\hline
\end{tabular}

\subsubsection{Functionality Cases}

We investigate two functionality cases. The objective is to examine the sensitivity of optimal facility design to specification of the functionality, and to uncertainty within each case. Table 5-2 summarizes the two cases. In Case 2, the required production rate is large in the last month of the production interval, and the required injection rate is also large in the last month of the injection interval. Clearly, various other scenarios could be investigated depending on the market being served, existing and potential competition, and other factors. These cases were defined so that the results would demonstrate the impact that functionality requirements have on the optimal configuration. 
Table 5-2 Summary of Functionality Requirements

\begin{tabular}{|l|l|l|l|l|}
\hline \multirow{2}{*}{ Month } & \multicolumn{2}{|l|}{ Functionality Case 1 } & \multicolumn{2}{l|}{ Functionality Case 2 } \\
\cline { 2 - 5 } & $\begin{array}{l}\text { Mean } \\
\text { (Bcf/month) }\end{array}$ & $\begin{array}{l}\text { Standard } \\
\text { Deviation } \\
\text { (Bcf/month) }\end{array}$ & $\begin{array}{l}\text { Mean } \\
\text { (Bcf/month) }\end{array}$ & $\begin{array}{l}\text { Standard } \\
\text { Deviation } \\
\text { (Bcf/month) }\end{array}$ \\
\hline 1 & -13.1 & 2.0 & -12.1 & 1.8 \\
\hline 2 & -15.4 & 2.3 & -12.4 & 1.9 \\
\hline 3 & -22.4 & 3.4 & -22.4 & 3.4 \\
\hline 4 & -21.6 & 3.2 & -25.4 & 3.8 \\
\hline 5 & -14.6 & 2.2 & -27.6 & 4.1 \\
\hline 6 & 13.7 & 2.1 & 13.7 & 2.1 \\
\hline 7 & 13.3 & 2.0 & 13.3 & 2.0 \\
\hline 8 & 17.9 & 2.7 & 17.9 & 2.7 \\
\hline 9 & 17.3 & 2.6 & 17.3 & 2.6 \\
\hline 10 & 11.5 & 1.7 & 10.5 & 1.6 \\
\hline 11 & 11.1 & 1.7 & 11.1 & 1.7 \\
\hline 12 & 2.1 & 0.3 & 16.1 & 2.4 \\
\hline
\end{tabular}

\subsubsection{Constants, Parameters, Variables and Decision Variables}

The main decision variables are the number of wells, compression horsepower, base gas, and production/injection rates. They are more important than the other variables because they directly contribute to the storage investment cost. The other decision variables are either strictly constrained by the reservoir dynamics or they are significantly affected by these decision variables. This implies that the decision maker cannot directly change the values of these variables. These implicit variables are called endogenous variables. The gas storage model includes the following endogenous variables: bottomhole and injection pressures, average reservoir pressure, individual flow rates, and cumulative production and injection rates.

\subsection{Asset-based problem: Deterministic Profit Maximization}

In asset based problems, the objective is to find the best design configuration and production-injection schedule that can maximize a performance attribute. The 
performance attribute can be defined in the form of total working gas volume, expected NPV or peak rate deliverability. If the NPV is selected, the objective function would appear as a profit, which includes the revenue terms are included and the objective function is in the general form of Profit $=$ Total Revenue - Total Cost. One might think that the cost minimization and profit maximization are the same, except for the formulation of the objective function. As we will see, this is not always true; there are several constraints that distinguish these two problems, especially in their application. In this section, we explain these differences.

The main feature of profit maximization is that the decision maker can determine the volume of the gas to be purchased or sold. For the cost minimization problem, a deterministic or probabilistic schedule should be satisfied, otherwise the proposed configuration would become noncompliant. In profit maximization, demand for gas is observed and the decision maker may or may not choose to satisfy the demand. However, there might be a chance to lose a potential gain or avoid a potential loss. One of the applications of the profit maximization problem is to answer the question of whether to develop a gas storage project or not, which is the basis of feasibility study and economic evaluation of projects. Table 5-3 compares the two problems. 
Table 5-3. Comparison between cost minimization and profit maximization problems

\begin{tabular}{|c|c|c|}
\hline Model Characteristic & Cost Minimization & Profit Maximization \\
\hline Objective/Application & $\begin{array}{l}\text { Optimum design configuration } \\
\text { Estimation of the cost of service }\end{array}$ & $\begin{array}{l}\text { Optimum design configuration } \\
\text { Feasibility study and economic } \\
\text { evaluation }\end{array}$ \\
\hline Strategy & $\begin{array}{l}\text { To find the best design } \\
\text { configuration to cover a } \\
\text { production - injection schedule } \\
\text { with minimum cost }\end{array}$ & $\begin{array}{l}\text { To find the best design } \\
\text { configuration and production - } \\
\text { injection schedule to maximize the } \\
\text { expected value of profit }\end{array}$ \\
\hline Objective Function & Minimize: Total Cost & $\begin{array}{l}\text { Maximize: Profit = Total Revenue - } \\
\text { Total Cost }\end{array}$ \\
\hline Constraints & $\begin{array}{l}\text { A deterministic or probabilistic } \\
\text { schedule should be satisfied, } \\
\text { otherwise the proposed } \\
\text { configuration becomes } \\
\text { incompliant }\end{array}$ & $\begin{array}{l}\text { A demand for gas may exist. } \\
\text { Depending on the strategy, the } \\
\text { decision maker may or may not } \\
\text { cover the demand. }\end{array}$ \\
\hline
\end{tabular}

In this section we present one sample of asset based problems, namely, deterministic profit maximization. The remainder of case studies is devoted to requirement based problems.

We have selected the relative NPV as the metrics to compare the results. As a diagnostic case we assume the parameters are deterministic as depicted in Table 54. As it can be seen the selling price and the purchase price have constant values over the whole cycle; this assumption is made for diagnostic purposes.

Table 5-4: Economic characteristics of the diagnostic case

\begin{tabular}{|c|c|}
\hline Characteristic & Assumed Value \\
\hline Purchase price & 3.5MM\$/Bcf \\
\hline Selling price & 7.5MM\$/Bcf \\
\hline Well cost & $10 \mathrm{MM} \$ /$ Well \\
\hline Compression cost & 2.5MM\$/MHP \\
\hline Bas gas cost & $3.5 \mathrm{MM} \$ / \mathrm{Bcf}$ \\
\hline Injection cost & $0.5 \mathrm{MM} \$ \mathrm{Bcf}$ \\
\hline Production cost & $0.5 \mathrm{MM} \$$ Bcf \\
\hline
\end{tabular}


For the market supply/demand we assumed values similar to the Functionality Case 2, which is shown in Table 5-5. Each period is one month. Positive values show the excess of gas in the market (supply) and negative values show the demand. As mentioned before, in profit maximization problems the solver is not constrained to cover the whole market demand. The solver has the choice to cover a portion of the demand, depending on the capability and potential benefits; however, it cannot exceed the market supply and demand.

Table 5-5 Supply/Demand for one cycle

\begin{tabular}{|c|c|}
\hline Month & Supply/Demand (Bcf) \\
\hline 1 & -12.1 \\
\hline 2 & -12.4 \\
\hline 3 & -22.4 \\
\hline 4 & -25.4 \\
\hline 5 & -27.6 \\
\hline 6 & 13.7 \\
\hline 7 & 13.3 \\
\hline 8 & 17.9 \\
\hline 9 & 17.3 \\
\hline 10 & 10.5 \\
\hline 11 & 11.1 \\
\hline 12 & 16.1 \\
\hline
\end{tabular}

Other parameters are kept the same as cost minimization problems. The results for the fixed number of 32 wells and fixed compression horsepower of 15.8MHP are summarized in Table 5-6. The volume of working gas is $82.9 \mathrm{Bcf}$ optimal NPV is -217.4MM\$. A negative NPV shows that the investment cannot payback all the expenses over one year. 
Table 5-6 15.8MHP option - Output results

\begin{tabular}{|l|c|}
\hline \multicolumn{1}{|c|}{ Output Parameter / Decision Variable } & Value \\
\hline Number of wells (fixed) & 32 \\
\hline Base gas volume (Bcf) & 29.3 \\
\hline Compression (MHP) - fixed & 15.8 \\
\hline Average CAPEX/ Working gas (MM\$/Bcf) & 5.58 \\
\hline Covered demand (Optimal working gas) & 82.9 \\
\hline NPV (MM\$) & -217.4 \\
\hline
\end{tabular}

The total production rate for each period would also be an interesting output because it can be used to determine the ratio of covered demand. As the results show, in the last period only $38 \%$ of the total demand is covered, which implies that it is not economical to cover the remainder of demand because of the compression and base gas requirements. An overall ratio of $83 \%$ of the total demand is covered.

Table 5-7 15.8MHP option - Covered demand

\begin{tabular}{|c|c|c|c|}
\hline Month & $\begin{array}{c}\text { Total production } \\
\text { (Bcf) }\end{array}$ & $\begin{array}{c}\text { Total Demand } \\
\text { (Bcf) }\end{array}$ & $\begin{array}{c}\text { Percent of } \\
\text { Total demand }\end{array}$ \\
\hline 1 & 12.1 & 12.1 & $100 \%$ \\
\hline 2 & 12.4 & 12.4 & $100 \%$ \\
\hline 3 & 22.4 & 22.4 & $100 \%$ \\
\hline 4 & 25.4 & 25.4 & $100 \%$ \\
\hline 5 & 10.6 & 27.6 & $38 \%$ \\
\hline Total & 82.9 & 99.9 & $83 \%$ \\
\hline
\end{tabular}

Another interesting factor would be the total storage ratio. This can be calculated as the ratio of total gas in storage during one cycle to the total storage capacity. For this case we have:

Total gas in storage $=82.9+29.3=112.2$

Total storage ratio $=112.2 / 140.6=79.8 \%$ 
The total storage ratio can be interpreted as a measure of effectiveness for the optimal configuration for the given model characteristics. This ratio can become more than one if the total number of storage cycles is more than one.

\subsubsection{Analysis of the Compression Horsepower}

Let us consider another case in which the both the compression horsepower and well count are fixed. For the sake of discussion, we assume the compression horsepower to be $20.64 \mathrm{MHP}$, which is similar to the cost minimization problems. We follow the same procedure; the results are given in Table 5-8.

Table 5-8 20.64MHP option - Output results

\begin{tabular}{|l|c|}
\hline \multicolumn{1}{|c|}{ Output Parameter / Decision Variable } & Value \\
\hline Number of wells (fixed) & 32 \\
\hline Base gas volume (Bcf) & 27.0 \\
\hline Compression (MHP) - fixed & 20.64 \\
\hline Average CAPEX/ Working gas (MM\$/Bcf) & 5.60 \\
\hline Covered demand (Optimal working gas) & 83.1 \\
\hline NPV (MM\$) & -219.7 \\
\hline
\end{tabular}

The covered demand has increased slightly (0.2Bcf). The base gas volume has decreased $(29.3-27=2.3 \mathrm{Bcf})$. So, we can see the trade-off between the base gas and compression. The ratio of average CAPEX per working gas volume increased, which shows that the CAPEX has increased. Comparing the two cases, the relative NPV is equal to $2.3 \mathrm{MM}$. So, we should expect that the 15.8MHP options would have a shorter discounted payback period. In fact the value of 15.8MHP was obtained by relaxing the compression horsepower bounds and allowing the the solver to find the optimal compression.

To verify the optimality of the proposed solution and to make our analysis more inclusive, the compression is increased to $25.64 \mathrm{MHP}$. The results are given in Table 5-9. The working gas is about the same value. The base gas has again decreased (1.8Bcf); however, it is not as much as the previous case. The NPV has decreased (5.3MM\$), which is consistent with expectations. 
Table 5-9 25.64MHP option - Output results

\begin{tabular}{|l|c|}
\hline \multicolumn{1}{|c|}{ Output Parameter / Decision Variable } & Value \\
\hline Number of wells (fixed) & 32 \\
\hline Base gas volume (Bcf) & 25.2 \\
\hline Compression (MHP) - fixed & 25.64 \\
\hline Average CAPEX/ Working gas (MM\$/Bcf) & 5.68 \\
\hline Covered demand (Optimal working gas) & 83.2 \\
\hline NPV (MM\$) & -225.0 \\
\hline
\end{tabular}

It is also required to check the compression below the suggested option to verify the optimality of the $15.8 \mathrm{MHP}$ option. For the case of $12 \mathrm{MHP}$, the results shown in Table 5-10 are obtained.

Table 5-10 12.0MHP option - Output results

\begin{tabular}{|l|l|}
\hline Output Parameter / Decision Variable & Value \\
\hline Number of wells (fixed) & 32 \\
\hline Base gas volume (Bcf) & 31.7 \\
\hline Compression (MHP) - fixed & 12.0 \\
\hline Average CAPEX/ Working gas (MM\$/Bcf) & 5.76 \\
\hline Covered demand (Optimal working gas) & 80.0 \\
\hline NPV (MM\$) & -225.3 \\
\hline
\end{tabular}

The NPV value has deteriorated, which implies that the suggested value by the solver is in fact the optimal solution. 


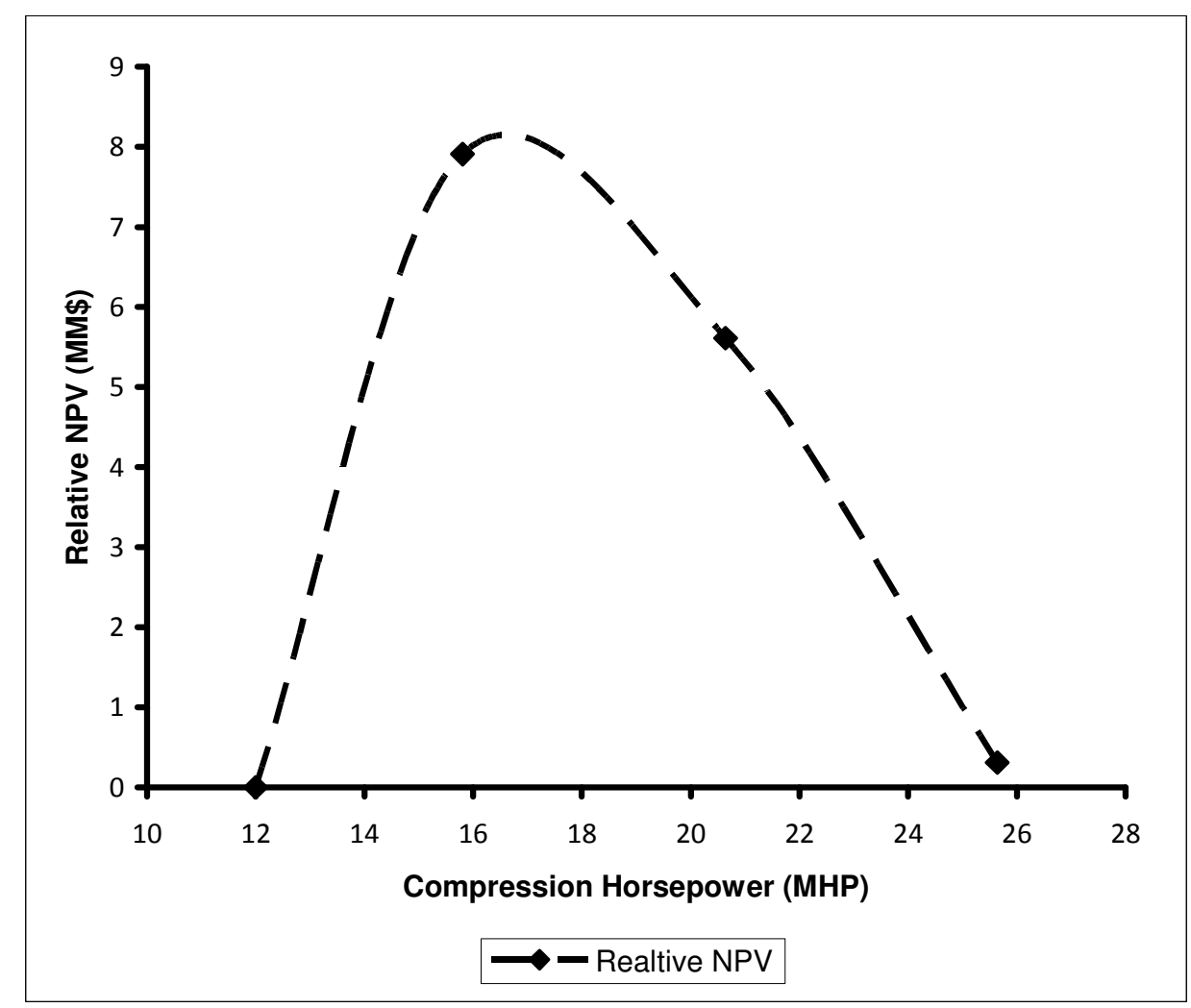

Figure 5-1 Relative NPV value for configuration of 32 wells

\subsubsection{Analysis of the Number of Wells}

Similar to the analysis performed for the compression, we can analyze the well count for the optimal value using the 3 compression horsepower options previously analyzed. We change the well count for each compression option to set up constant compression curves (the same as cost minimization problems). To keep the results brief and manageable, we summarize the NPV values for each configuration, which are presented in Figure 5-2. We selected the configuration of 35 wells and $12 \mathrm{MHP}$ as the reference NPV, other NPVs are calculated with respect to this value. In this case, the well cost is relatively high, and the NPV value is very sensitive to the well count. Figure 5-3 shows the behavior of NPV around the optimal point. It can be seen that the configuration of 20 wells and $15.8 \mathrm{MHP}$ is optimal for the current model assumptions. 


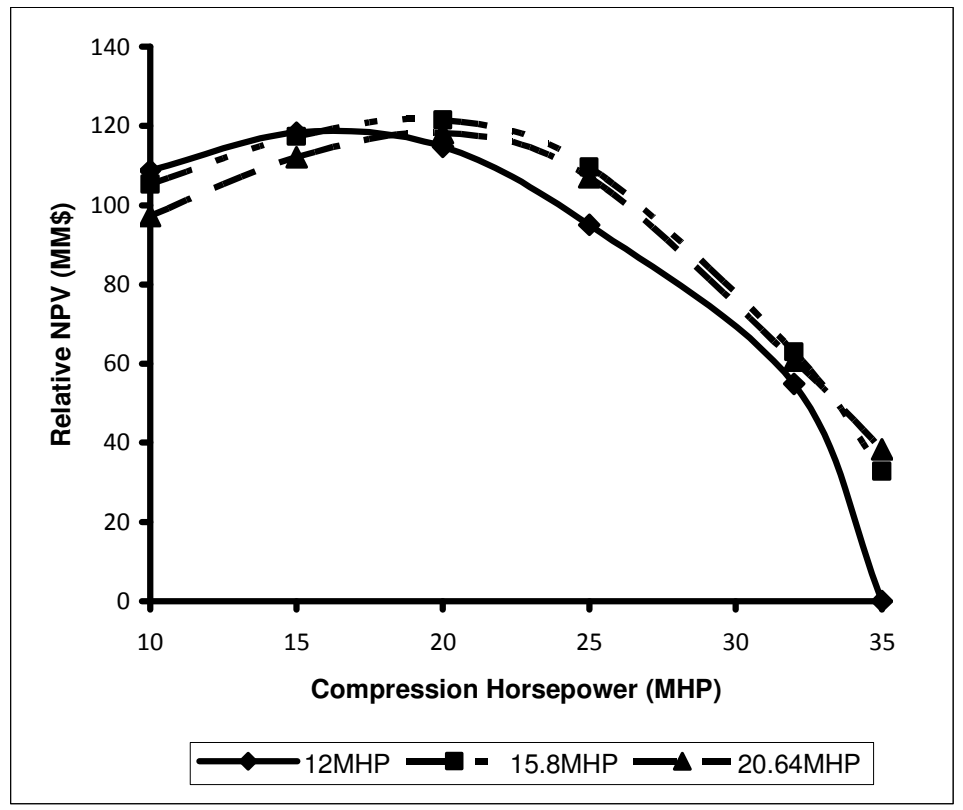

Figure 5-2 Relative NPV values for different well count and compression options

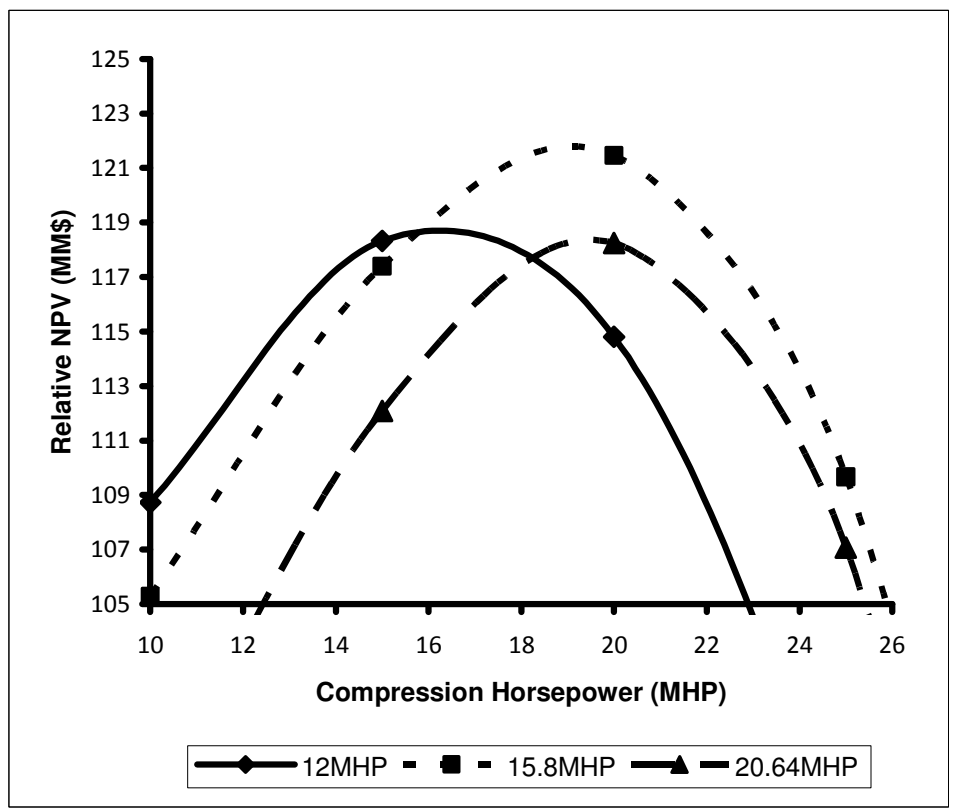

Figure 5-3 Relative NPV values for different well count and compression options - around the optimal point 


\subsection{Requirement-based Problems}

\subsubsection{Problem A: Deterministic Analysis for Functionality Requirements}

Before an uncertainty analysis, it is instructive to conduct a deterministic analysis as a reference case. Also, the deterministic analysis can be used to validate expectations about how the modeled system should respond in different circumstances, and this is easier to accomplish without the added complexity of uncertainty. Both functionality cases were examined using the workflow to model various combinations of well counts and horsepower.

Basic results from the analysis are presented in Figures 5-4 and 5-5. Each curve represents a constant level of horsepower, and the cost is plotted as a function of well count. For Functionality Case 1 depicted in Figure 5-4, the optimal combination is 37 wells with $20.64 \mathrm{MHP}$ compression and base gas equal to $33 \%$ of total storage capacity. For Functionality Case 2 depicted in Figure 5-5, the optimal combination is 35 wells with $25.64 \mathrm{MHP}$ compression and base gas equal to $35 \%$ of total storage capacity (although the option of 43 wells and $30.64 \mathrm{MHP}$ yields a very similar cost). These results show the sensitivity of optimal configuration (wells and horsepower) to the deterministic functionality requirements. In each case, there are configurations which are clearly suboptimal. In practice, the operator could use these results to reduce the number of configurations to carry forward into detailed engineering. 


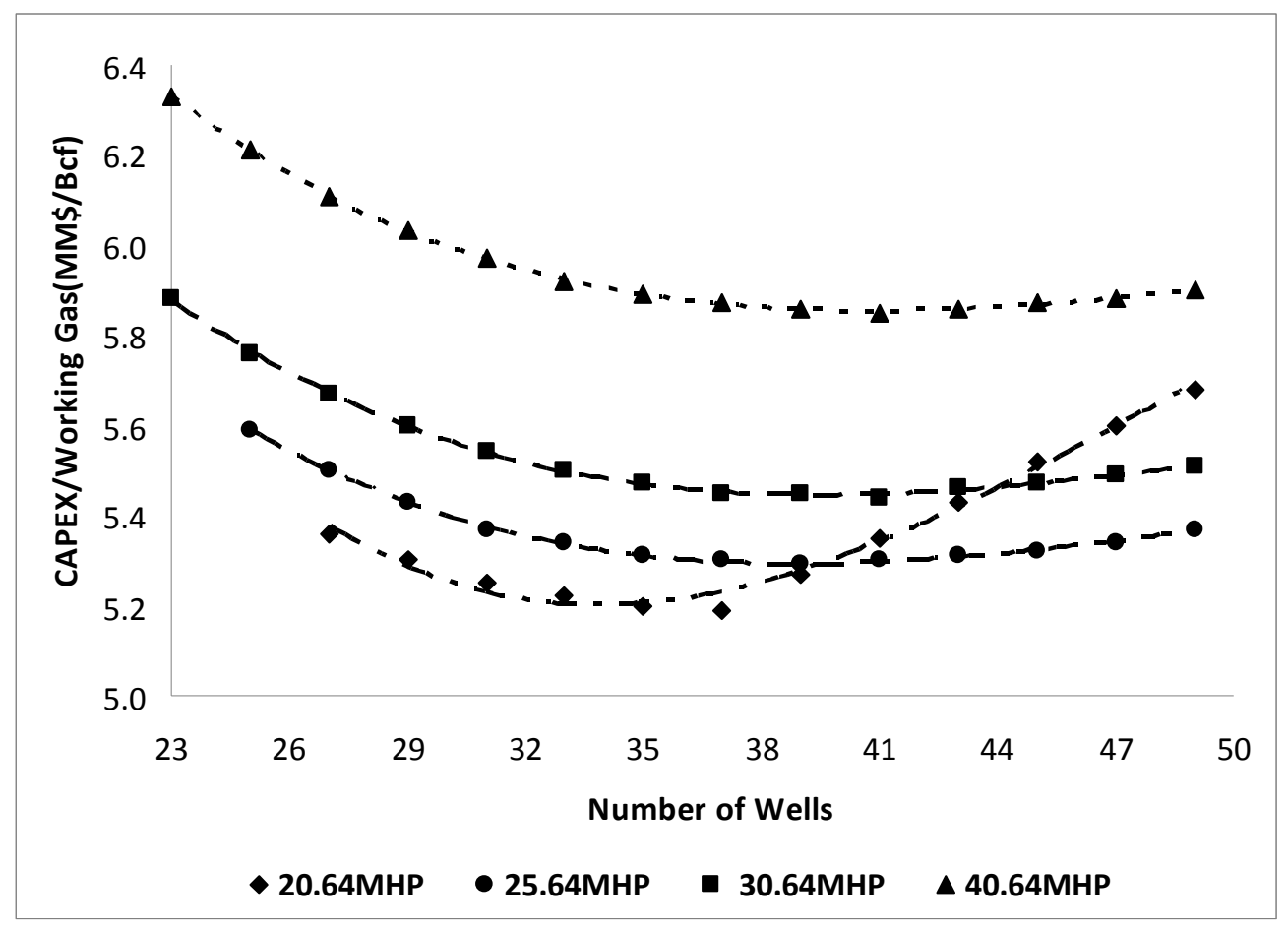

Figure 5-4 Cost Curves for Functionality Case 1

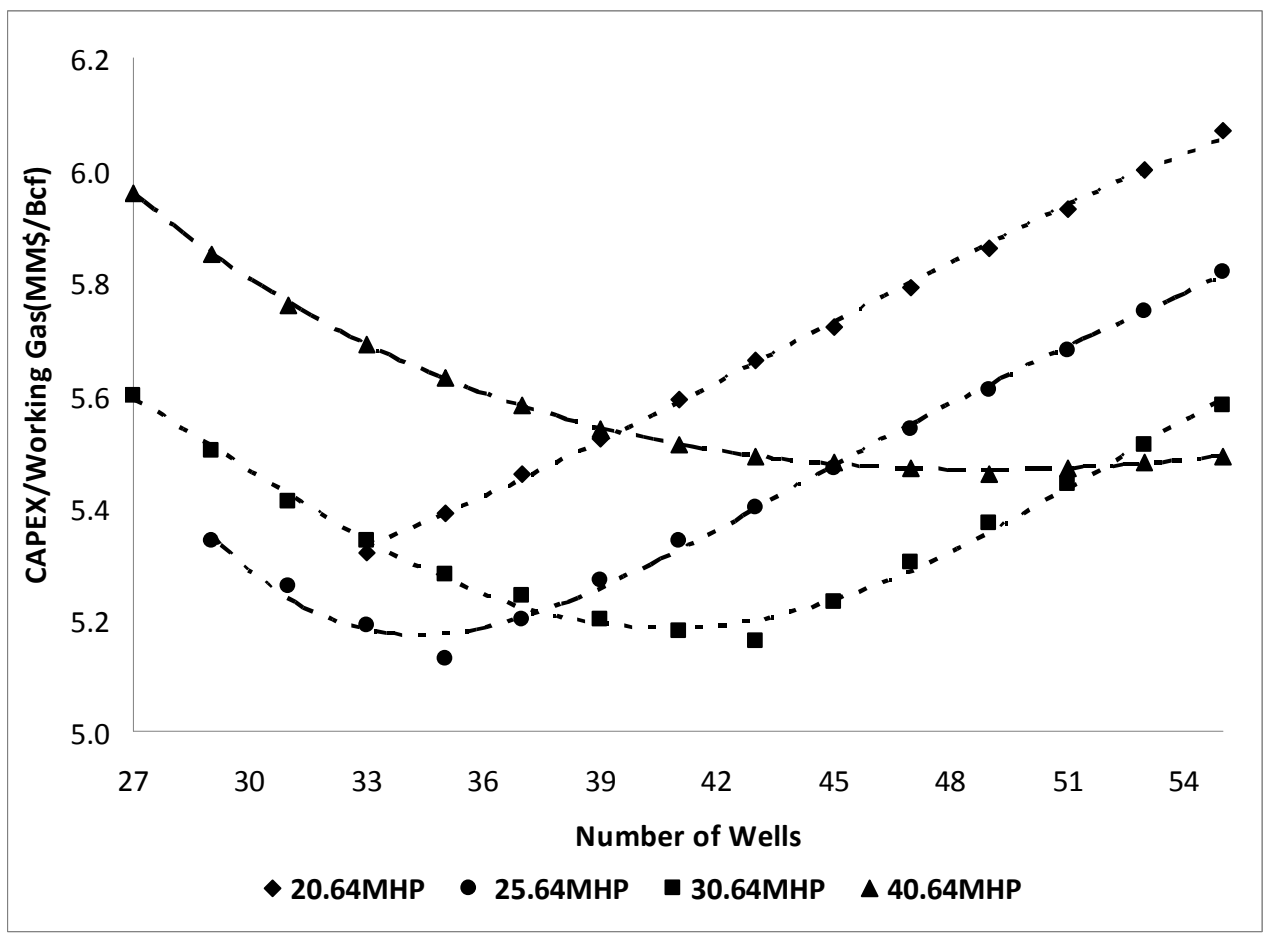

Figure 5-5 Cost Curves for Functionality Case 2 
As the number of wells increases, the volume of base gas decreases because more wells can produce at a lower rate and pressure to satisfy the requirements; this can be observed in Figures 5-6 and 5-7. For constant horsepower, the capital tradeoff is between base gas cost and well cost. At low well counts, there is a net benefit to additional drilling, but eventually the cost of additional wells outweighs the savings in base gas cost. This tradeoff is clear when comparing the cases of 25.64 MHP and 30.64 MHP for Functionality Case 2 in Figures 5-5 and 5-7; the two low cost solutions represent different well counts and base gas, yet yield similar costs. This tradeoff typically results in U-shaped cost curves. But this tradeoff is not always possible. In Figure 5-7, observe the near linear relationship for the case of 20.64 MHP for Functionality Case 2. This result occurs because the base gas cannot be reduced and still satisfy the functionality requirements, thus, additional wells only add to cost.

As horsepower increases, the volume of base gas generally decreases because of increased injection capacity, but depending on the well cost, fewer or more wells may be optimal. When well cost is small, it is generally optimal to increase the well count and decrease the base gas volume. But there is a limit to this effect because sufficient base gas is required to provide the minimum reservoir energy for production; hence, at some point, we expect that increasing horsepower would not affect the optimal number of wells. This result is observed for Functionality Case 1 in Figure 5-4 where the optimal well count is approximately the same for horsepower greater than or equal to $25.64 \mathrm{MHP}$.

The shape of the curves is also important information. In Figure 5-4, it is observed that three of the cost curves are somewhat flat to the right of the minimum. In cases where increased functionality might be desirable (e.g. Functionality Case 2), this kind of information would allow the operator to err in the direction of additional wells with very little downside risk (depending on the horsepower installed). 


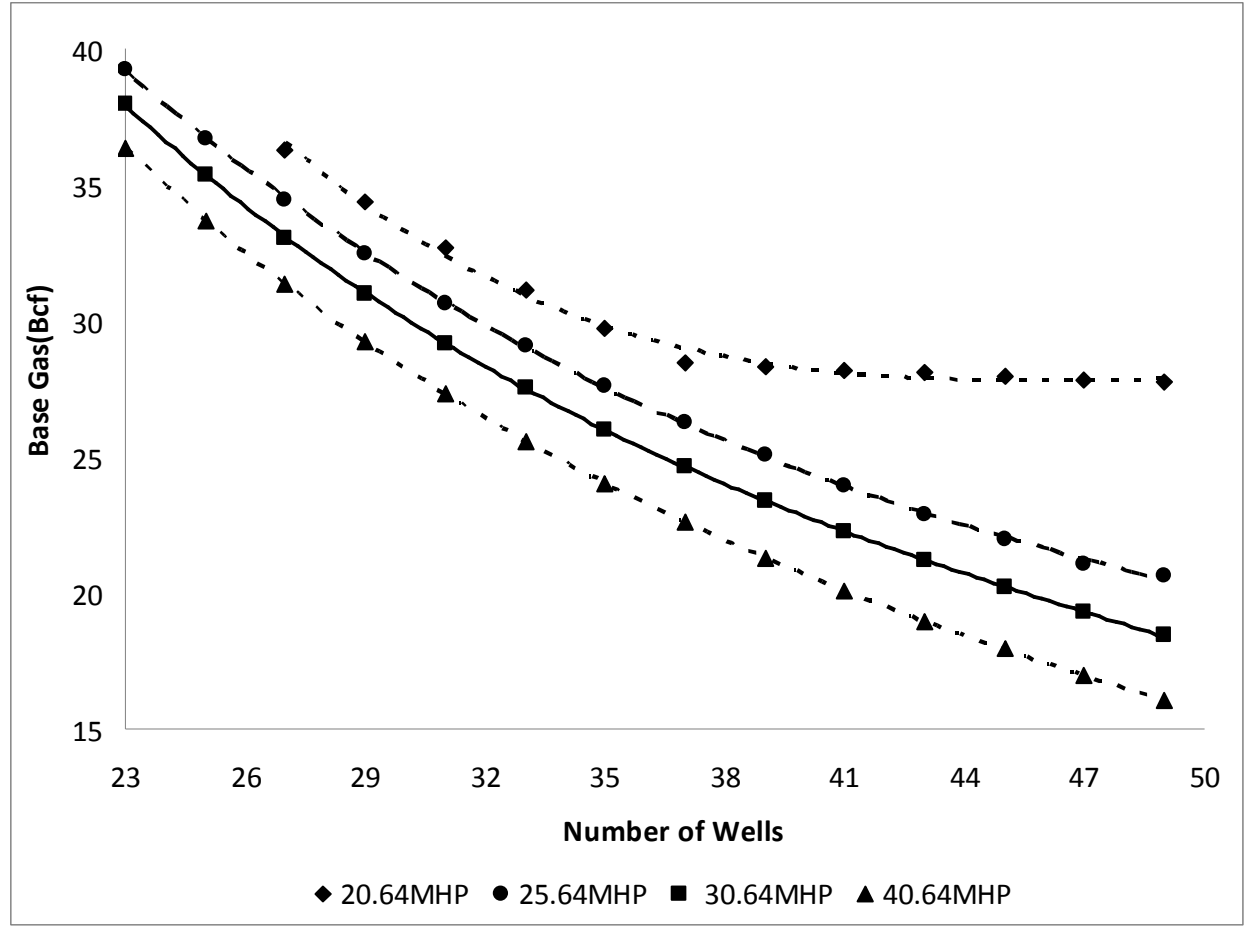

Figure 5-6 Optimal Base Gas for Functionality Case 1

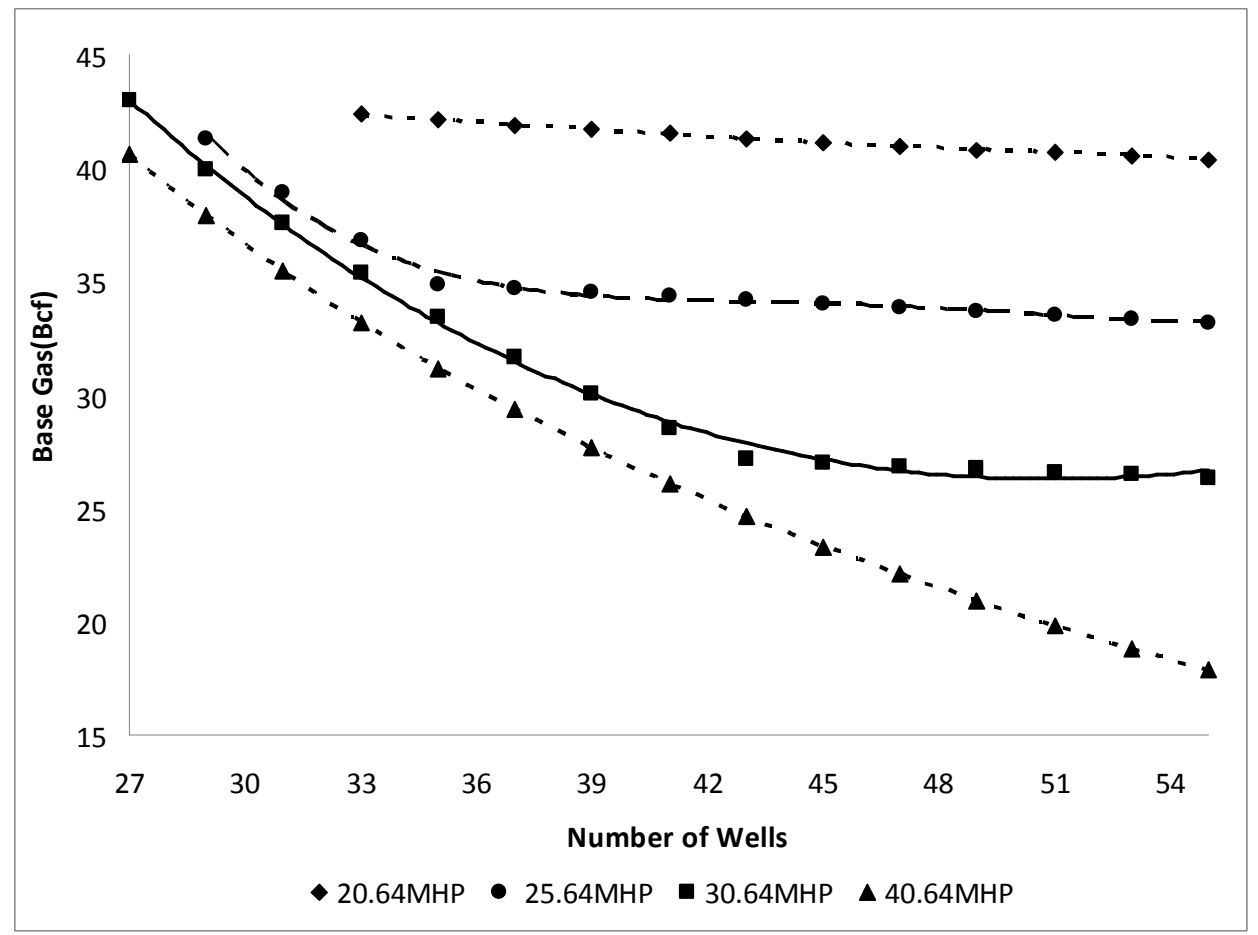

Figure 5-7 Optimal Base Gas for Functionality Case 2 


\subsubsection{Problem B: Uncertainty Analysis for Functionality Requirements}

We now extend the analysis of Problem $A$ to include uncertainty in the functionality requirements. That is, the objective is to evaluate the effect of uncertainty in functionality on the optimal configuration. The requirement in each month is specified as a normal probability distribution with mean and standard deviation as shown in Table 5-2. Here, we present the results from an analysis of Case 2 using 40 iterations.

Figure 5-8 presents the PDF of working gas that results from the repeated sampling of the Case 2 functionality requirements. Figure 5-9 presents the associated PDF of CAPEX/Working Gas cost for two facility configurations. Table 5-11 summarizes the major output CAPEX/Working Gas stats for the two configurations. Following the workflow, similar PDFs are constructed for other facility configurations as was done deterministically in Problem $A$.

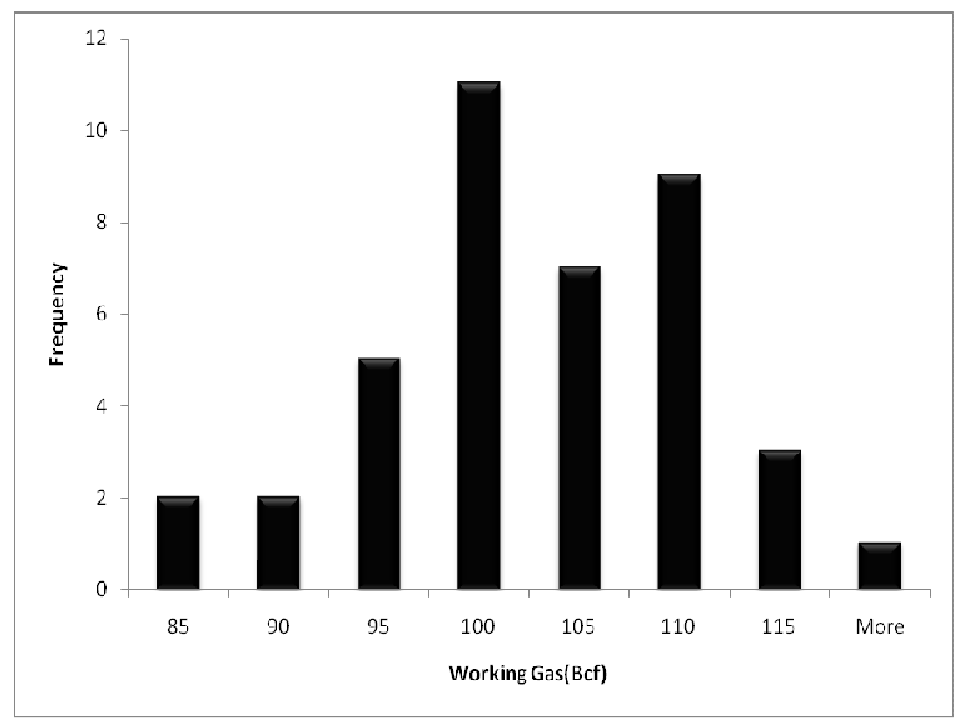

Figure 5-8 PDF of CAPEX/Working Gas for Functionality Case 2: 40 wells, 25.64 MHP 
(a) 40 Wells

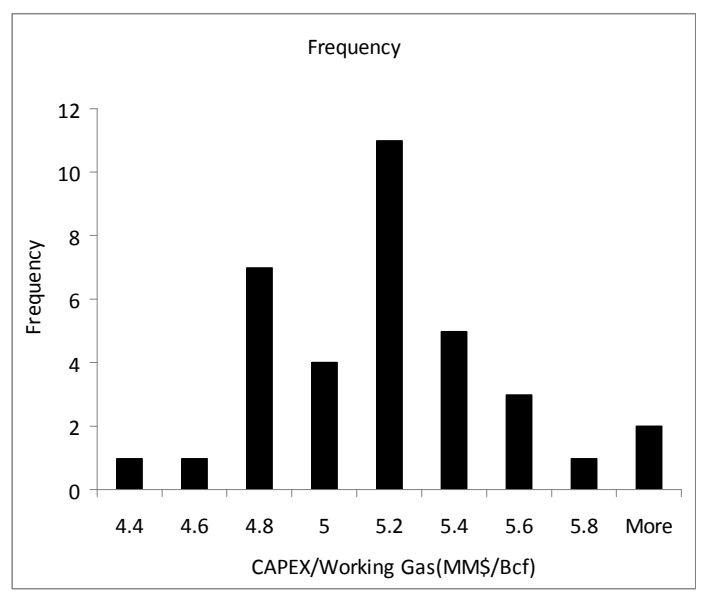

(b) 45 Wells

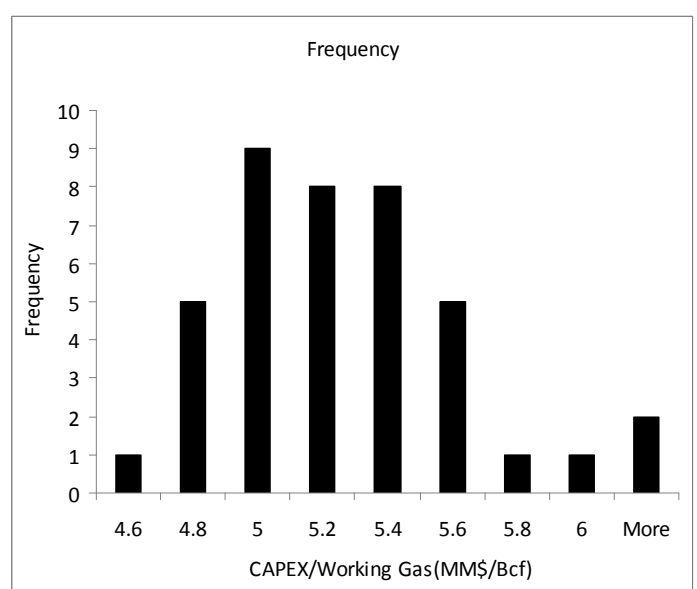

Figure 5-9 of Working Gas for Functionality Case 2

Table 5-11 Statistical parameters for 25.64MHP and different well counts

\begin{tabular}{|c|c|c|c|}
\hline $\begin{array}{c}\text { Statistical } \\
\text { Parameter }\end{array}$ & 35 Wells & 40 Wells & 45 Wells \\
\hline Average & 5.17 & 5.075 & 5.16 \\
\hline STD & 0.327 & 0.345 & 0.378 \\
\hline P90 & 5.575 & 5.489 & 5.605 \\
\hline P10 & 4.807 & 4.707 & 4.715 \\
\hline
\end{tabular}

\subsubsection{Compliance}

On the surface, the uncertain case appears no different from the deterministic case except for the change in output from a single value to a PDF, and it seems logical to proceed as before and plot the results and determine the optimal configuration. However, there is an underlying problem in this case, and in general. Because the well count and horsepower are fixed in the short term, certain realizations of the functionality requirements will not be technically feasible. That is, the base gas cannot be increased enough to satisfy all constraints in the optimization 
model. For example, in the case of Figure 5-9, only 35 of the 40 iterations, or $87.5 \%$, were feasible and included in the histogram. We refer to this result as " $87.5 \%$ compliant." The sample mean is computed based on the 35 feasible solutions.

In light of the compliance issue, caution is required in interpreting results. It must be recognized that infeasible iterations occur when the gas storage system is exposed to extreme realizations of the uncertain variables. To make such iterations feasible would require changes to the facility and impose additional costs. By excluding these iterations, the sample mean will be biased. Therefore, cost metrics are only reliable for comparison when compliance is at or close to $100 \%$. For example, in Figure 5-10 we present the result of an analysis for the case of 25.64 MHP. Each data point represents the sample mean of the feasible outcomes; while it is not shown on these figures, the standard deviation could also be plotted on these axes. As before, a tradeoff between the well count and base gas is observed that yields an apparent optimal well count at 40-42 wells. However, the right y-axis indicates the compliance rate, and it is clear that these cases are not $100 \%$ compliant, and therefore the cost metric is biased.

Relationships can be estimated for other horsepower levels and the results compared, and an example of this is provided in Figure 5-11. The compliance rates are noted on the individual curves. For a given horsepower, the compliance rate increases with increasing well count. In the case of 20.64 MHP however, there is a physical limit which constrains the compliance rate to $85 \%$ regardless of the number of wells. While caution is required in interpretation of the cost output, the compliance information is very helpful in observing system behavior and gaining insight into the tradeoffs and cost impacts of variance in the uncertain variables. In some cases, the output can be used to estimate the incremental cost of compliance (when bias can be assumed to be small). This format of output facilitates communication within the project team and with managers and other stakeholders. 


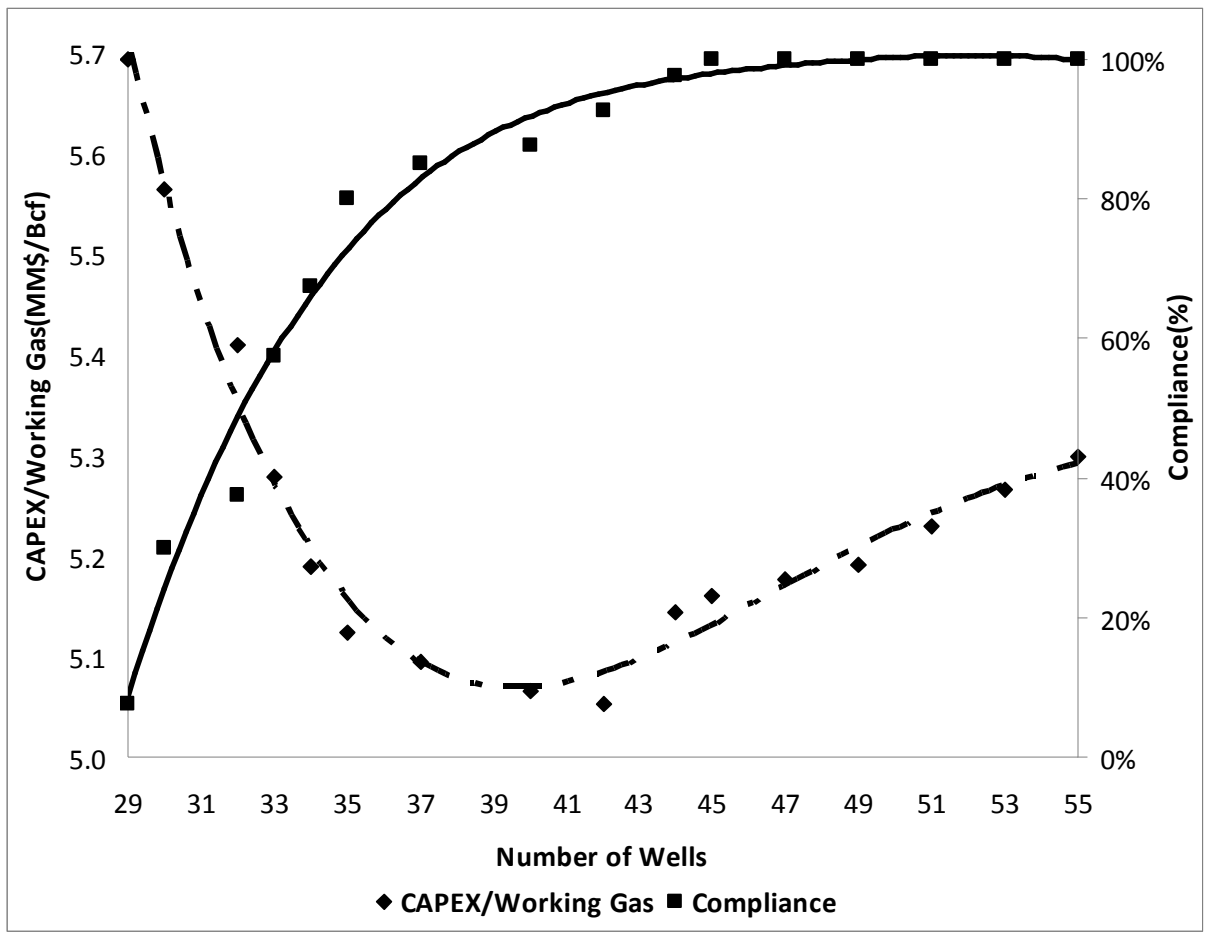

Figure 5-10 Cost and Compliance Curves for Functionality Case 2: 25.64 MHP

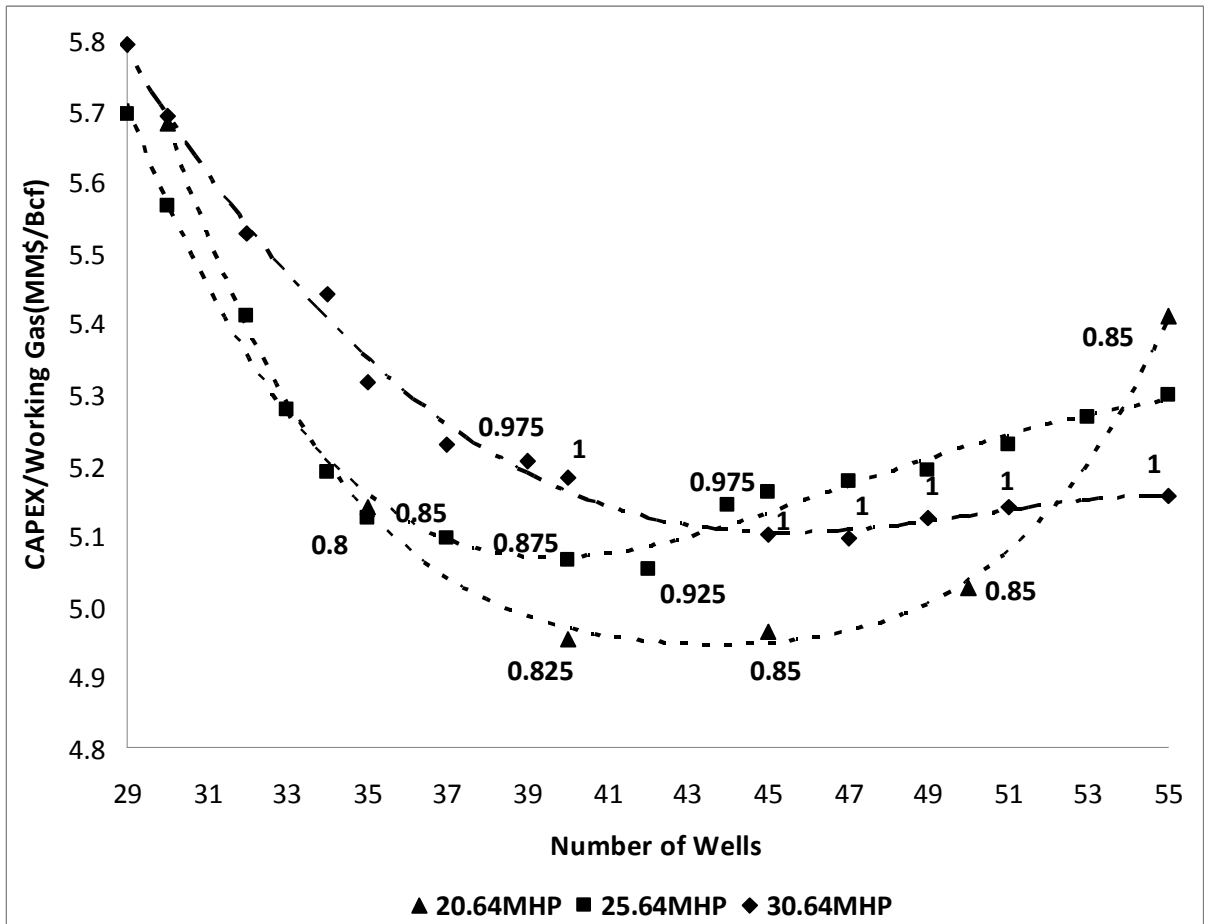

Figure 5-11 Cost and Compliance Curves for Functionality Case 2: multiple MHP 


\subsubsection{Problem C: Uncertainty Analysis for Reservoir Properties}

In addition to uncertainty in the functionality requirements, we investigate the impact of uncertainty in reservoir properties on the optimal facility configuration. ${ }^{8}$ In this section, we assess the impact of uncertainty in permeability. It is assumed that the average permeability is measured in millidarcies (md) and is normally distributed with a standard deviation of $10 \mathrm{md}$. Three cases (means) are defined for analysis: a base case $(50 \mathrm{md})$, an optimistic case $(60 \mathrm{md})$, and a pessimistic case $(40 \mathrm{md})$. The functionality is specified deterministically in all cases using Functionality Case 2 (see Table 5-2). Figure 5-12 summarizes the cost and compliance results for the base case permeability distribution.

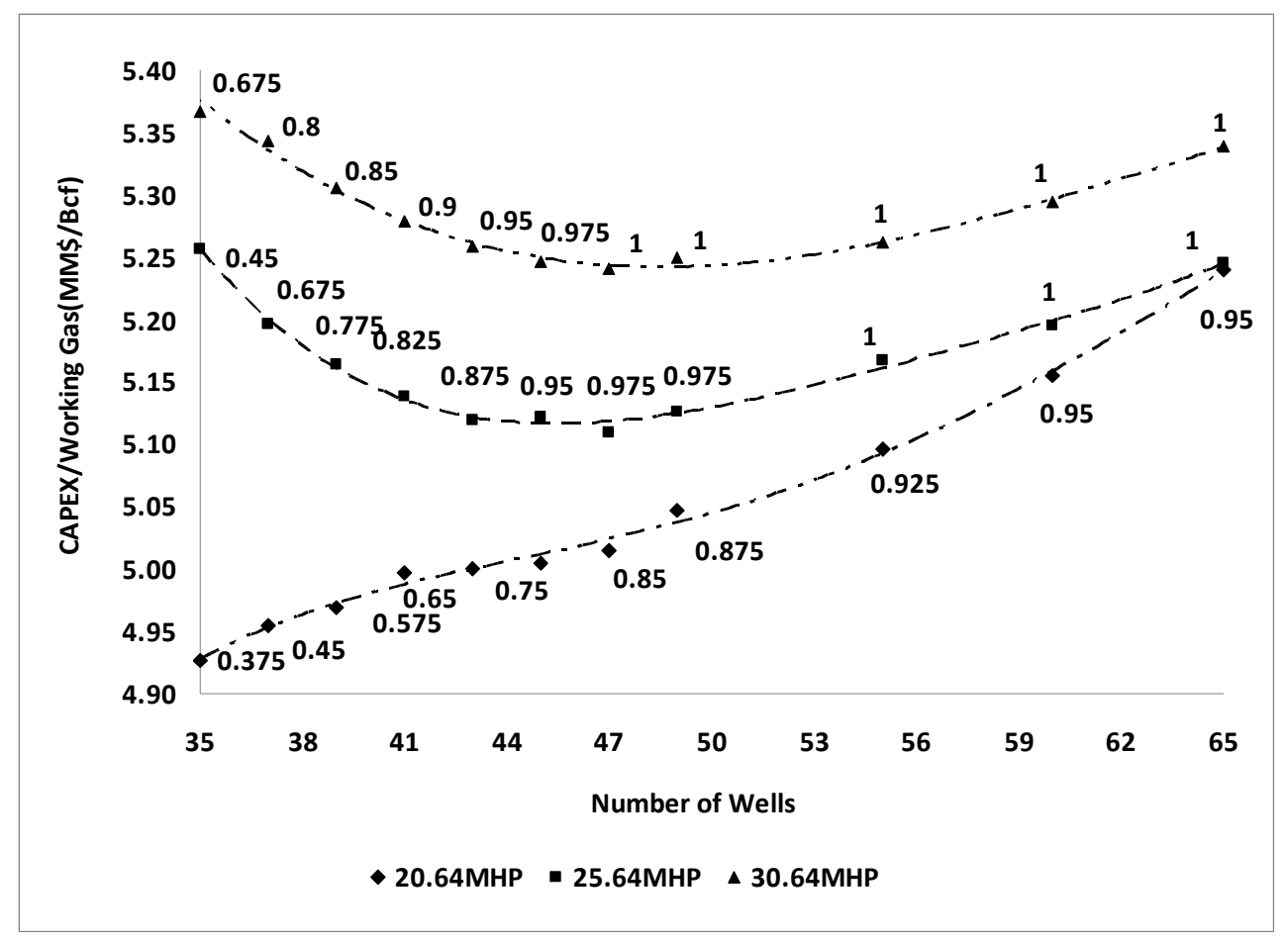

Figure 5-12 Cost Curves and Compliance for Base Case Permeability Distribution: multiple MHP

These results can be compared to the analysis of uncertainty in functionality. For example, consider the configuration of 25.64 MHP and 43 wells in Figure 5-11. This configuration is expected to be $95 \%$ compliant with an expected cost of

\footnotetext{
${ }^{8}$ A deterministic analysis of the impact of reservoir properties on gas storage reservoir performance has been investigated by Bilgesu and Ali (2004).
} 
$\sim 5.1 \mathrm{MM} \$$ /Bcf of working gas. However, based on the uncertainty analysis of permeability in Figure 5-12, the same configuration (with deterministic functionality) is only $\mathbf{8 7 . 5 \%}$ compliant and may entail a slightly higher cost. For this configuration, the uncertainty in permeability introduces greater variability into the performance of the system. Conclusions regarding cost differences cannot be as definitive because of the imperfect compliance in both cases. When the curves in Figure 5-12 are evaluated in light of the compliance rate, and the focus is placed on results with $\sim 100 \%$ compliance, it is observed that the $30.64 \mathrm{MHP}$ configuration achieves full compliance with the least number of wells (as expected); however, the difference in compliance between this option and the 25.64 MHP configuration is minor. The 20.64 MHP option does not achieve full compliance. This type of information can be used by the project team and decision makers to determine the configuration for the facility. ${ }^{9}$ In this case, the 25.64 MHP option appears to yield the lowest expected cost.

\subsubsection{Sensitivity Analysis of Model Outputs}

The purpose of this section is to illustrate the effect of variation in permeability as an uncertain input parameter on the investment cost as an output parameter.

The procedure is as follows: Two configurations are selected: 45 wells with 20.64MHP compression and 45 wells with 25.64MHP compression and the output results for investment cost and base gas volume are analyzed. The permeability is assumed to have a normal distribution with the 95\% confidence interval of [30 70] md (base case). For each configuration 40 samples for permeability are taken and the model is run two find the optimal cost and compliance ratio. Figure 5-14 shows the results for investment cost versus the permeability observations. The

configuration of 45 wells with $20.64 \mathrm{MHP}$ is $80 \%$ compliant and 45 wells with 25.64MHP is $95 \%$ compliant and the standard deviation for the output CAPEX/Working gas is equal to 0.28 and $0.34 \mathrm{MM} \$$ /bcf, respectively (see Tables 5-12

\footnotetext{
${ }^{9}$ To do so would require estimates for the costs of non-compliance. These costs are idiosyncratic and no attempt was made to include them in the current investigation.
} 
and 5-13 for the input and output stats). As it can be observed the standard deviation is not small, which shows the sensitivity of the investment requirements with respect to the permeability. Figure 5-13 shows that the investment cost monotonically decreases with increasing the permeability.

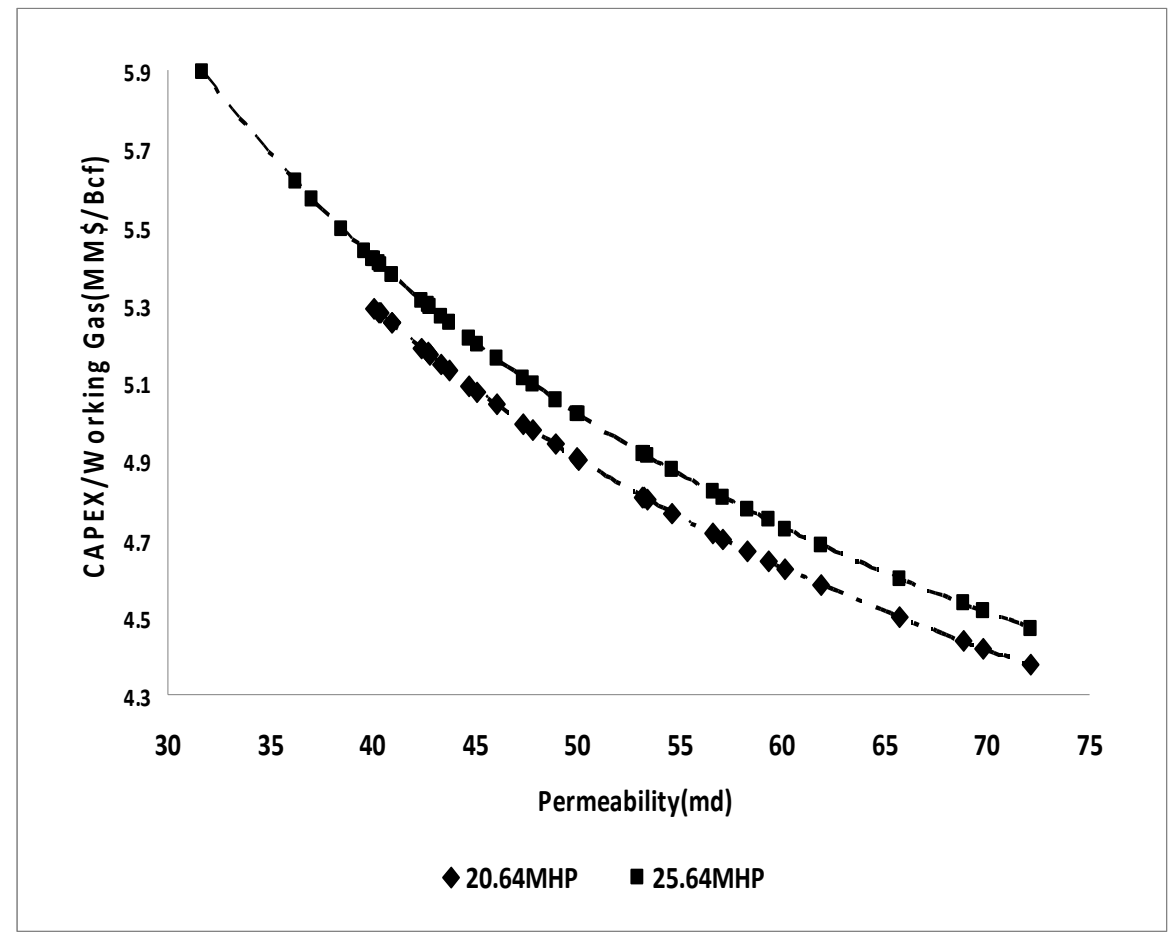

Figure 5-13 CAPEX/WG - Permeability Correlation - 45 Wells (Around the optimal configuration for 25.64MHP option)

Table 5-12 provides the general stats for the observed permeability values for 40 iterations. Table 5-13 shows the general output statistics for the compliant cases. The unit for permeability is milidarcy, the unit of CAPEX/WG is MM\$/Bcf.

Table 5-12 Permeability Stats - All Observations

\begin{tabular}{|l|l|}
\hline Min Observed & 28.97 \\
\hline Max Observed & 72.13 \\
\hline Average & 48.40 \\
\hline STD & 10.80 \\
\hline P10 & 38.32 \\
\hline P90 & 62.25 \\
\hline
\end{tabular}


Table 5-13 Output Statistics

\begin{tabular}{|c|c|c|}
\hline & 20.64MHP & 25.64MHP \\
\hline Number of iterations & 40 & 40 \\
\hline Infeasible & 8 & 2 \\
\hline Compliance (\%) & 0.8 & 0.95 \\
\hline \multicolumn{3}{|c|}{ CAPEX/Working Gas Stats } \\
\hline Min & 4.37 & 4.47 \\
\hline Max & 5.29 & 5.90 \\
\hline Average & 5.00 & 5.11 \\
\hline STD & 0.28 & 0.34 \\
\hline P10 & 4.51 & 4.67 \\
\hline P90 & 5.25 & 5.45 \\
\hline \multicolumn{3}{|c|}{ Permeability Stats - Feasible Cases } \\
\hline Min Observed & 40.05 & 31.70 \\
\hline Max Observed & 72.13 & 72.13 \\
\hline Average & 51.06 & 48.92 \\
\hline STD & 9.40 & 10.07 \\
\hline P10 & 40.91 & 39.40 \\
\hline P90 & 64.94 & 62.64 \\
\hline \multicolumn{3}{|c|}{ Base gas Stats- Feasible Cases } \\
\hline Min Observed & 21.92 & 19.70 \\
\hline Max Observed & 34.98 & 40.02 \\
\hline Average & 29.57 & 28.81 \\
\hline STD & 4.00 & 4.82 \\
\hline P10 & 23.91 & 22.51 \\
\hline P90 & 34.42 & 33.65 \\
\hline
\end{tabular}




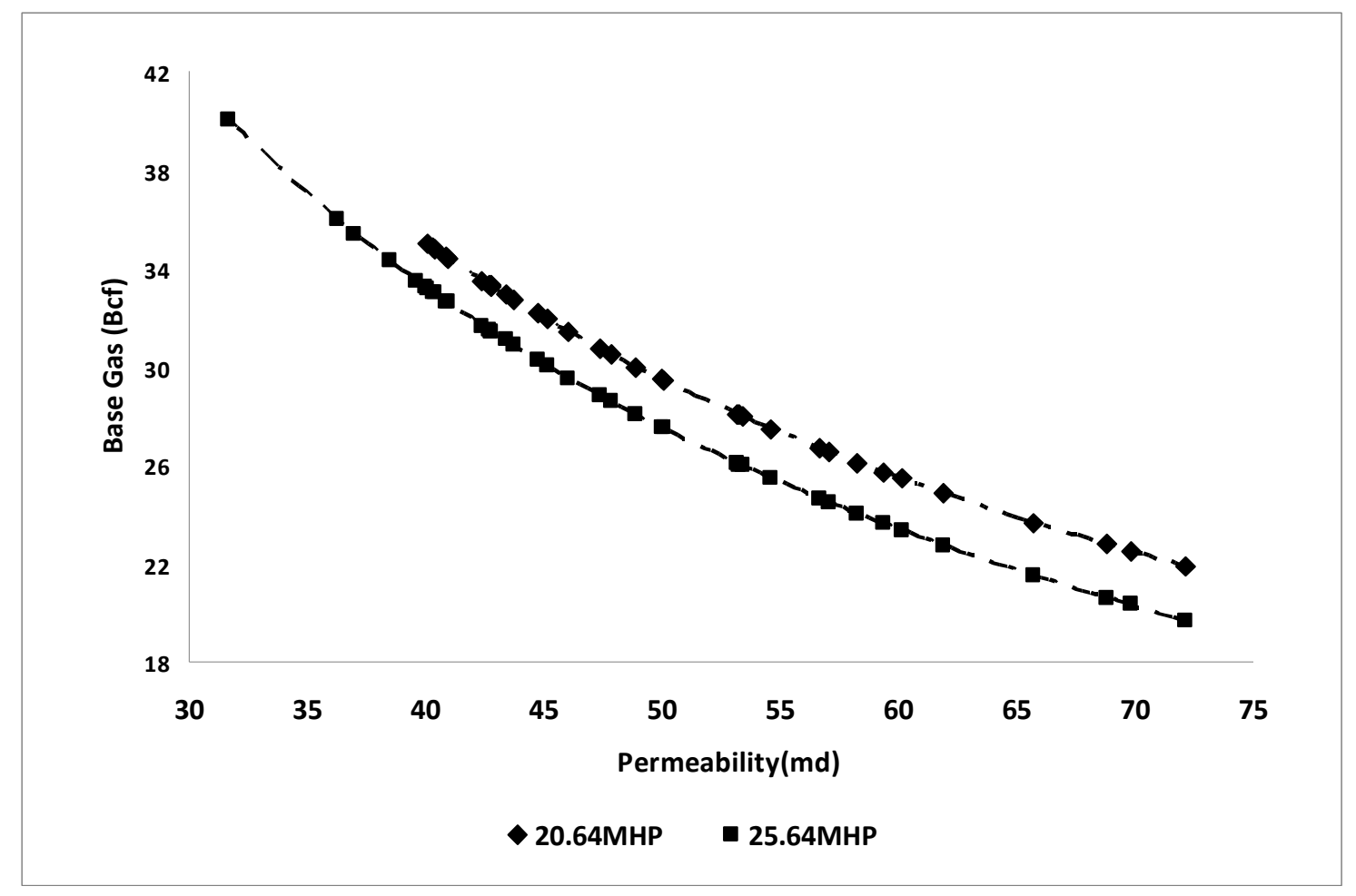

Figure 5-14 Base gas - Permeability Correlation - 45 Wells

Considering the results provided in Figures 5-13 and 5-14, along with Tables 512 and $5-13$, the following observations can be made:

1. The minimum permeability observed in the sample is $28.97 \mathrm{md}$. However, the minimum observed permeability that resulted in a compliant output is 40.05md for 45wells \& 20.64MHP and 31.70md for 45wells \& 25.64MHP, respectively. Therefore, minimum permeability of $40 \mathrm{md}$ for a $20.64 \mathrm{MHP}$ option is required to fully cover Functionality Case 2; the minimum permeability reduces to $31.70 \mathrm{md}$ for the $25.64 \mathrm{MHP}$ option. This shows that compression can act against the effects of poor permeability and maintain the compliance of the whole system.

2. Comparing the standard deviation of optimal investment cost, the configuration of 45 wells \& $25.64 \mathrm{MHP}$, has a larger standard deviation than 45 wells $\& 20.64 \mathrm{MHP}$. This is because of the compliance. The configuration of $45 \mathrm{~W} \& 25.64 \mathrm{MHP}$ is more compliant, which means it is able to cover more 
extremely low outcomes of permeability. This requires more investment and hence the range of observed investment costs becomes broad. In other words, the standard deviation increases.

3. Regarding the [P10 P90] interval for the compliant outcomes of permeability, it can be observed that for the 45W-20.64MHP configuration, the compliant permeability interval has shifted to the right compared to the $45 \mathrm{~W}$ 25.64MHP. This is because of non-compliant outcomes of permeability are omitted from the calculation of permeability interval. Similar analysis can be made to justify the interval shift for the observed optimal CAPEX/ Working gas values of the configuration. For this case the [P10 P90] interval for 45W25.64MHP configuration has shifted to the right.

The correlation between the base gas volume and permeability is also worth of investigation, which is shown in Figure 5-14. The general trend is the same as expected: as the reservoir permeability increases, lower base gas volumes are required to cover given functionality requirements. Also, there is a trade-off between the base gas volume and the compression horsepower, as we increase one, the other can be reduced. So, we expect the base gas volume for 25.64MHP configurations to lie below the corresponding values for 20.64MHP configurations. Obviously these trends apply to a certain range.

Referring to Table 5-13, it can be observed that there are 8 permeability outcomes for which the $45 \mathrm{~W}-20.64 \mathrm{MHP}$ option is noncompliant, while this is only 2 for the case of $45 \mathrm{~W}-25.64 \mathrm{MHP}$ option. It would be instructive to investigate the reasons for this difference.

We know that for a fixed well count, the effect of low permeability can be balanced by increasing the compression horsepower and the base gas volume. There are 6 permeability observations for which the 45W-20.64MHP is noncompliant while the $45 \mathrm{~W}-25.64 \mathrm{MHP}$ is compliant. This implies that for these 6 observations, the increase in horse power would be necessary to cover the functionality requirements and it cannot be replaced by an increase in base gas. For the remaining outcomes for 
which both configurations are compliant, the base gas volume and compression are interchangeable; however, the increase in compression is more costly compared to the base gas.

The tradeoff between the base gas volume and compression can be summarized as follows: When there is a capacity to cover the demand using the current compression potential, the solver selects to use that; when the compression capacity reaches its limits, the model starts to increase the base gas volume, until it cannot be increased anymore due to the reservoir capacity and functionality requirements; obviously, beyond that point, the model becomes infeasible or noncompliant.

\subsubsection{Sensitivity Analysis of Permeability Distribution}

The uncertainty analysis can be repeated for the optimistic and pessimistic permeability distributions. Compliance curves for the three permeability cases and 25.64 MHP are shown in Figure 5-15. For this level of horsepower in the pessimistic case, the system only achieves $85 \%$ compliance; higher levels of horsepower would be needed to increase this rate. The lowest well count for full compliance is observed in the optimistic case. 


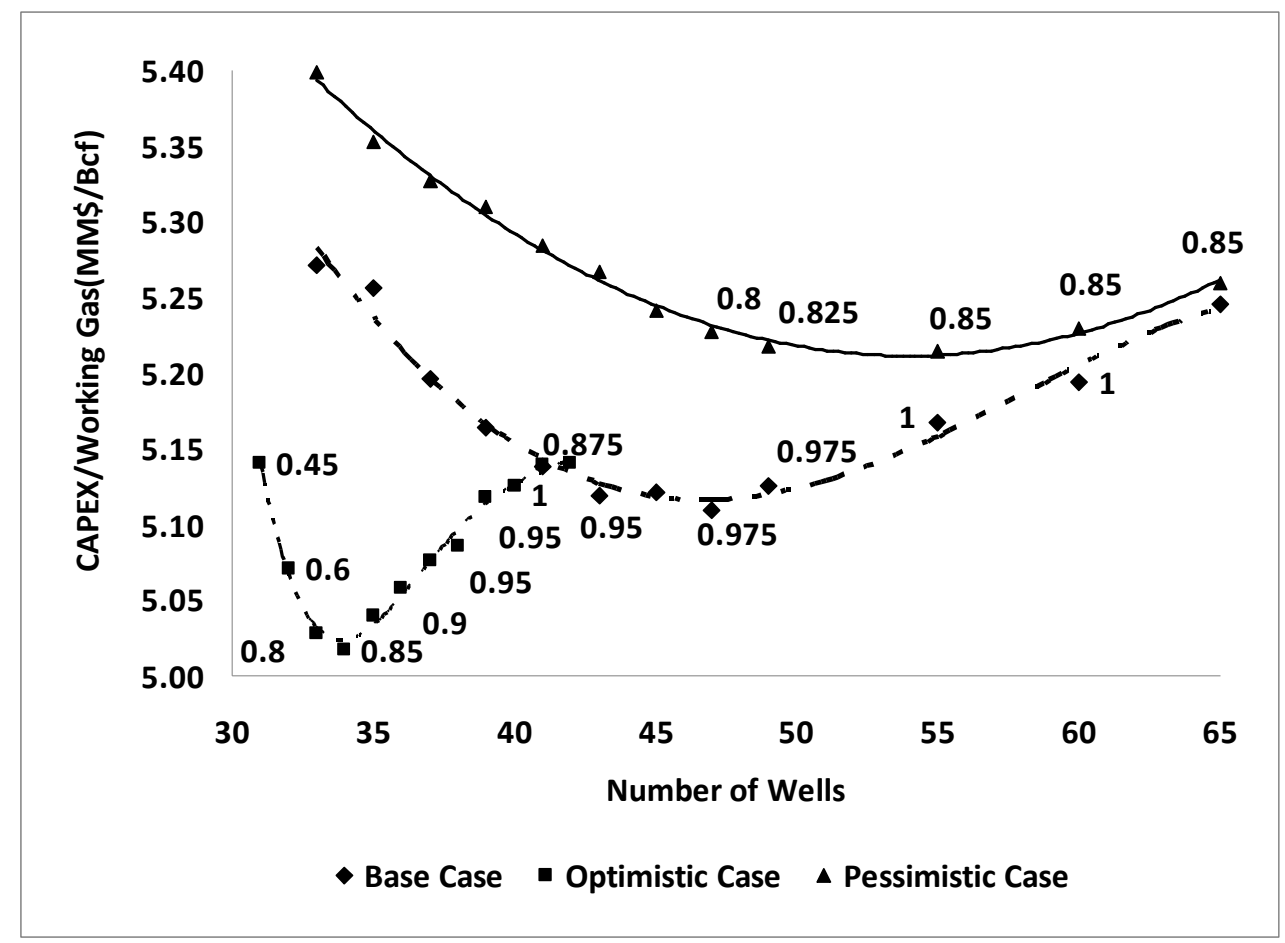

Figure 5-15 Cost Curves and Compliance for Three Permeability Distributions (25.64MHP)

\subsubsection{Problem D: Joint Uncertainty in Permeability \& Functionality Requirements}

In the analysis of Problems B and C, only one uncertain estimate was evaluated while other estimates were specified deterministically. But one of the motivations for the proposed workflow and optimization model is to enable a comprehensive uncertainty analysis where several assumptions are specified probabilistically. When several assumptions are specified in this way, we refer to the problem as joint uncertainty analysis. In this section, we investigate a problem where the functionality requirements and permeability are both specified probabilistically. ${ }^{10}$ In all cases analyzed below, we assume Functionality Case 2 and the base case for the permeability distribution.

We selected the permeability as an uncertain subsurface parameter and functionality requirements as a surface parameter. Functionality Case 2 and the base

\footnotetext{
${ }^{10}$ We also assume that these variables are statistically independent. For combinations of other variables, it may be more appropriate to specify dependence among the variables.
} 
case for the permeability were selected. The probability distribution for these parameters was assumed to be the same as problems $B$ and $C$. The objective is to find those combinations that yield the minimum required compliance with the minimum investment cost. Each data point presented in Figure 5-16 represents the results of optimization procedure for one possible configuration of compression horsepower and well count. Each configuration was tested for 40 randomized combinations of permeability and functionality requirements. The production and injection rates and the bottomhole pressures for each well, the injection pressure and the base gas volume were allowed to change based on the model response to the outcome of permeability and functionality requirements.

The combined compliance - cost curves for each of the three compression options are shown in Figure 5-16. Considering the same well count, any possible configuration based on the 20.64MHP compression yields a lower investment cost and lower compliance ratio, compared to the corresponding 25.64MHP option. The results of analysis for the well counts of more than 49 showed that the compliance ratio for these options cannot increase anymore while the investment cost continues to increase. Note that the $20.64 \mathrm{MHP}$ option is at most $85 \%$ compliant, while the 25.64MHP option is at most $95 \%$ compliant.

Regarding the investment cost, observe that although the 20.64MHP option always yields lower costs, it does not cover challenging outcomes of functionality and permeability. As a result, these challenging outcomes will not be included in the average cost calculations. If there were penalties associated with the non-compliant outcomes, the average cost for both options would have proportionally increased.

Similar to the previous problems, the cost and compliance values for different compression horsepower are shown in Figure 5-16. In this case, the combined effect of joint variability in functionality and permeability is to increase the minimum number of wells needed for full compliance (when this is possible), and to increase 
costs. ${ }^{11}$ In the case of $30.64 \mathrm{MHP}$, full compliance is now reached with 55 wells (with a cost metric of $\sim \$ 5.50)$ whereas in Problem $B$ this was 39 wells $(\$ 5.20)$ and in Problem $C$ this was 47 wells $(\$ 5.25)$. In the other two horsepower options full compliance is not achieved. The 20.64 MHP option approaches a limit of $85 \%$ compliant while the 25.64 MHP option approaches 95\% compliant. As before, the curves help decision-makers understand system performance and can be used to value investments in increased compliance.

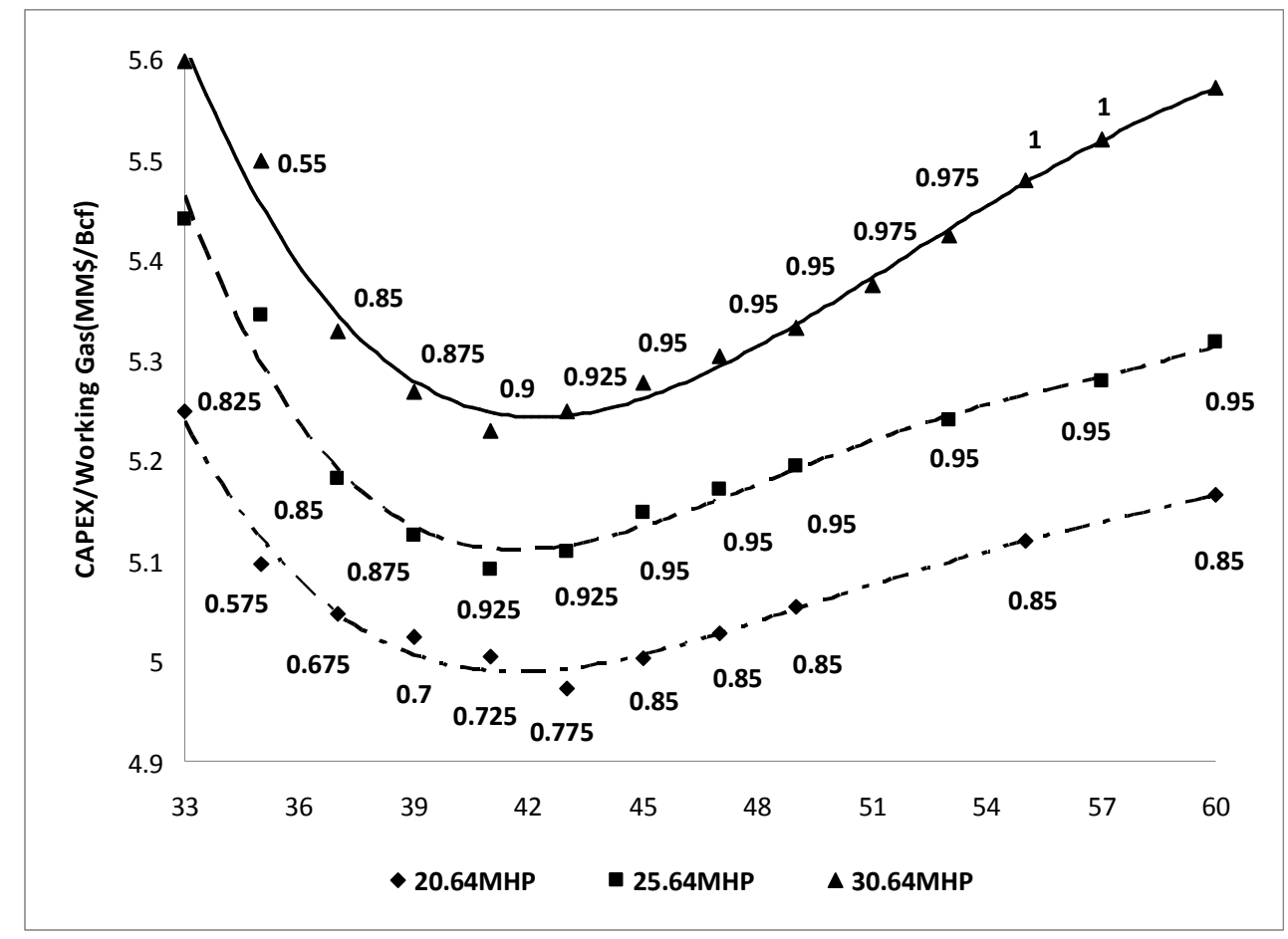

Figure 5-16 Cost Curves and Compliance for Joint Uncertainty Analysis: multiple MHP

In order to select the best design candidate(s), we can divide the configurations into two main categories: configurations with relatively low investment cost and low compliance and configurations with relatively large investment cost and large compliance. Comparing these two groups, one can infer

\footnotetext{
${ }^{11}$ However, because there are two independent and uncertain inputs, it is possible that in alternate specifications these deleterious effects will be reversed and the joint uncertainty will improve system performance.
} 
that in order to select the appropriate concept, there are two possible strategies to follow: one is to choose the highly compliant configuration (for example 95\%) and accept the relatively large investment cost, or to take the low cost configuration and accept the consequences of lost demands (non-compliant outcomes). Those following the first strategy might argue that the cost of lost demand (penalty) is not considered in the calculations for the non-compliant options; those who select the second strategy, on the other hand, might say that the priority is to keep the costs as low as possible and the lost demand should be covered with short term resources. Obviously, the choice depends on the long term policy of the decision makers.

Introducing the uncertainty to the input parameters can cause the optimal solution to shift towards more conservative and more costly configurations, namely, those with larger potential capacity. This observation can be justified by considering the fact that in any optimization process, the last option for the solver is to render the model as infeasible, which is equivalent to a non-compliant outcome. Because infeasibility is an undesirable outcome, the solver searches for more costly options with larger potential capacities. This effect can be seen by comparing the results of the joint uncertainty problem with the corresponding deterministic model (problem A). We can observe that the average investment cost is in the same range for both problems (see Figures 5-5 and 5-16), which suggests that the average base gas volume is almost the same for both deterministic and probabilistic solutions. The well count for the minimum cost, however, is different. The minimum investment cost occurs for a lower well count in the deterministic problem compared to the probabilistic problem and the probabilistic cases are not fully compliant.

The optimal solutions for the deterministic and probabilistic problems are not considerably different. This is because the whole gas storage design has a high degree of flexibility so that it provides the opportunity for the trade-offs between the well count, compression horsepower and the base gas volume. The metrics used to compare the configurations (investment cost/volume of working gas) has also a low degree of resolution. This means that the total investment cost is divided by the 
total volume of working gas, which is around $100 \mathrm{Bcf}$. As a result, a minor difference of $0.01 \mathrm{MM} \$ / \mathrm{Bcf}$ of working gas is equivalent to $1 \mathrm{MM}$ investment cost.

As we compare the results of the joint uncertainty problem with the results of single uncertainty in functionality requirements (problem $B$ ), we realize that the compliance ratio of joint uncertainty is less than the single uncertainty for a fixed configuration, which is expected. The average investment cost is almost in the same range. The optimal solution is in the range of 39 and 45 wells. These observations show that compared to the uncertainty in functionality requirements, the uncertainty in permeability does not have a significant effect on the optimal cost; however it has caused the compliance to decrease. 


\title{
6. Summary, Conclusions and Recommendations for Future Work
}

\begin{abstract}
A systematic and comprehensive uncertainty analysis can help operators make better gas storage facility design decisions. In this thesis, an uncertainty analysis workflow and integrated optimization model was proposed and demonstrated. The approach simultaneously accounts for reservoir behavior, facility options, and economic objectives. This approach balances the level of accuracy and the computational effort and speed. In addition to improving decision making, using this or similar workflows and models in the screening and concept selection stages of gas storage projects should facilitate uncertainty analysis and communication of results within the project team, with managers, and with other stakeholders.
\end{abstract}

\subsection{Concluding Remarks}

1. The reservoir tank model has been integrated with facility and economic models to evaluate different design configurations. This model is fast, which allows for the evaluation of many competing options.

2. The level of detail included in the model depends on the availability of information, significance of the details, time limitations and computational effort required.

3. Use of simple models can provide a general idea about the range of optimal expected values for the major decision variables such as the 
well count and the facility functionality. These models are easy to develop, and the output is easy to analyze.

4. The methods applied to uncertainty analysis commonly require a large amount of computational effort. Because of the limitations of time, information and computational resources, it may not be feasible to couple fine grid reservoir simulators with uncertainty analysis workflows. I such cases, the use of simple models is preferred over the fine-grid reservoir simulators.

5. Uncertainty in the estimates can have a significant impact on the optimal facility configuration. The concept of compliance is proposed and analyzed for the subsurface and market uncertainties. The tradeoffs between the major decision variables and the corresponding thresholds are analyzed.

6. The optimization model specified in GAMS is fast-solving and eliminates most practical constraints on the scope of the uncertainty analysis. It is a powerful tool that can help to describe the potential outcomes for various facility configurations and to identify and evaluate tradeoffs between competing options.

\subsection{Recommendations for Future Work}

1. The current model specified a single tank reservoir. All reservoir properties are represented by average values. One may extend the current model to account for multiple compartments and/or multiple layers.

2. One of the assumptions for the current model is that the reservoir size is fixed and the minimum functionality requirements are defined; i.e. the storage asset is already selected and based on the given constraints of reservoir and market the best configuration is determined. This approach can be extended to evaluate several storage candidates for a 
potential market and find the best storage reservoir to cover a predicted demand. Also, for a given reservoir, several functionality requirements (representing several potential markets) can be evaluated to maximize the net present value gained over the storage cycles.

3. The proposed approach for the uncertainty analysis is a general way to think about optimization under uncertainty. It does not define a specific procedure to evaluate a configuration under uncertainty. The current procedure uses a basic Monte Carlo sampling approach. There are other potential approaches such as importance sampling or control variates, which can be applied to solve the problem. Exact solution methods such as the Benders decomposition method can also be used to solve the universal problem. One avenue for investigation would be to compare different sampling techniques and compare the resulting optima.

4. The gas storage model has assumed single phase flow of natural gas. However, there is a potential to add a set of equations for modeling multiphase flow (e.g. as would be observed in aquifers). Multiphase tank models could also be employed to investigate other problems such $\mathrm{asCO}_{2}$ storage in aquifers. If the equations for $\mathrm{CO}_{2}$ miscibility in depleted oil reservoirs can be specified with sufficient accuracy, then these storage systems can also be optimized using the proposed approach for uncertainty analysis.

5. In cases where the reservoir is too complex for the use of tank models, it is appropriate to use fine grid reservoir simulation. However, these more complex simulators often force a reduction in the scope of the uncertainty analysis, and are more cumbersome when managerial flexibility is desired to be incorporated. Often, a response surface workflow is employed to investigate the uncertainty. One issue worthy 
of future research is to develop a systematic comparison of simple (tank) models versus complex (fine-grid and response surfaces) models to examine the incremental value of complexity in simulation. That is, we expect the fine grid simulator to be more accurate in cases of reservoir heterogeneity, but are the project valuation and design decisions significantly different than those that would have been made using a simpler model? This type of research project would attack the commonly held notion that accuracy has value-there may be cases where this is simply not true. 


\section{Appendix A: Gas Storage Code in GAMS}

The following code is used to analyze gas storage facility design under uncertainty. This is a nonlinear programming problem. The objective is to find the best configuration to satisfy a functional requirement.

Facing uncertain subsurface parameters and uncertain demand, decisions about the well count, compression horsepower and base gas volume need to be made. The Monte Carlo crude sampling method is used for uncertainty analysis. For more information about the assumptions and results, refer to:

Ettehadtavakkol, A., Jablonowski, C.J., Lake, L.W. Gas Storage Facility Design under Uncertainty. Thesis SPE 123987-MS. Presented at the SPE Annual Technical Conference and Exhibition, New Orleans, Louisiana, USA, October 4-7, 2009

Gas Storage Facility Design Under Uncertainty (Version 6.5)

SETS

i month/m1*m12/

ifirst(i) first period

ilast(i) last period

Coldmonths(i)

Hotmonths(i)

$\mathrm{W}$ producers or injectors /W1*W35/

* W injectors /Injwell1*Injwell4/

nh number of horsepower sets /nh1*nh3/

n number of trials $/ \mathrm{n} 1 * \mathrm{n} 40 /$

;

ifirst $(\mathrm{i})=$ yes $\$(\operatorname{ord}(\mathrm{i})$ eq 1$)$;

ilast $(\mathrm{i})=$ yes $\$(\operatorname{ord}(\mathrm{i})$ eq $\operatorname{card}(\mathrm{i}))$;

Coldmonths(i) $=$ yes $\$((\bmod (\operatorname{Ord}(\mathrm{i}), 12)$ le 3$)$ or $(\bmod (\operatorname{ord}(\mathrm{i}), 12)$ ge 11$))$;

Hotmonths(i) $=$ yes $\$($ not Coldmonths $(i))$;

*alias( W, Wprime);

display coldmonths, hotmonths;

scalars 
$* * * * * * * * * * * * * * * * * * * * * * * * * * * * * * * * * * * * * * * * * * * * * *$

* Gas Reservoir Data

************************************************

* Pinit Reservoir pressure at $\mathrm{i}=0$ in psia /1200/

PMax Maximum reservoir pressure in Mpsia/2.600/

PZMax Mpsia/3.1413/

PMin Minimum reservoir pressure in Mpsia/0.400/

PZMin Mpsia/0.52728/

* GB Base Gas capacity in MSCF /6.15769E+05/

GMax Original gas in place in Bcf/140.6203/

GMin Bcf/12.4828/

* PiZi Pi over Zi ratio in psi /1546/

* InitialGas Initial gas at $\mathrm{i}=0$ in MScf /2.869086E+06/

phi Porosity /0.15/

ZMax compressibility factor at PMax/0.7532/

PVInc ratio in PV increace /1.0/

$* * * * * * * * * * * * * * * * * * * * * * * * * * * * * * * * * * * * * * * * * * * * * *$

* Producer/Injector Data

$* * * * * * * * * * * * * * * * * * * * * * * * * * * * * * * * * * * * * * * * * * * * * *$

$\mathrm{h}$ Total thickness in $\mathrm{ft} / 20 /$

$\mathrm{k}$ Permeability in $\mathrm{md} / 50 /$

$\mathrm{T}$ Temperature in $\mathrm{R} / 600 /$

Re Drainage radius in $\mathrm{ft} / 7447.3 /$

A Total reservoir area in acres /6096.0/

CA Shape factor /30.6/

$\mathrm{Rw}$ Wellbore radius in $\mathrm{ft} / 0.25 /$

S Skin Factor /-4/

Pwfmin Minimum well flowing pressure Mpsi/0.150/

Pwinjmax Maximum well injecting pressure Mpsi/2.600/

MuZavgpro Average(Viscosity*compression) factor /0.01345/

* MuZavginj Average(Viscosity*compression) factor /0.01386/

* DrawdnPlm Maximum pressure drawdawn in Mpsi /0.300/

* InjPlm Maximum injection pressure diffrence Mpsi /0.300/

qlm Maximum withdrawal rate in MMscf per day /35/

Ilm Maximum injection rate in MMscf per day /35/

Pexp pressure exponent /0.7/

***************************************************

* Compression Data

$* * * * * * * * * * * * * * * * * * * * * * * * * * * * * * * * * * * * * * * * * * * * *$

PPr Pipeline pressure in Mpsi /1.00/

ComCost Capital compression cost MMdollar per Mhorsepower /5/ 


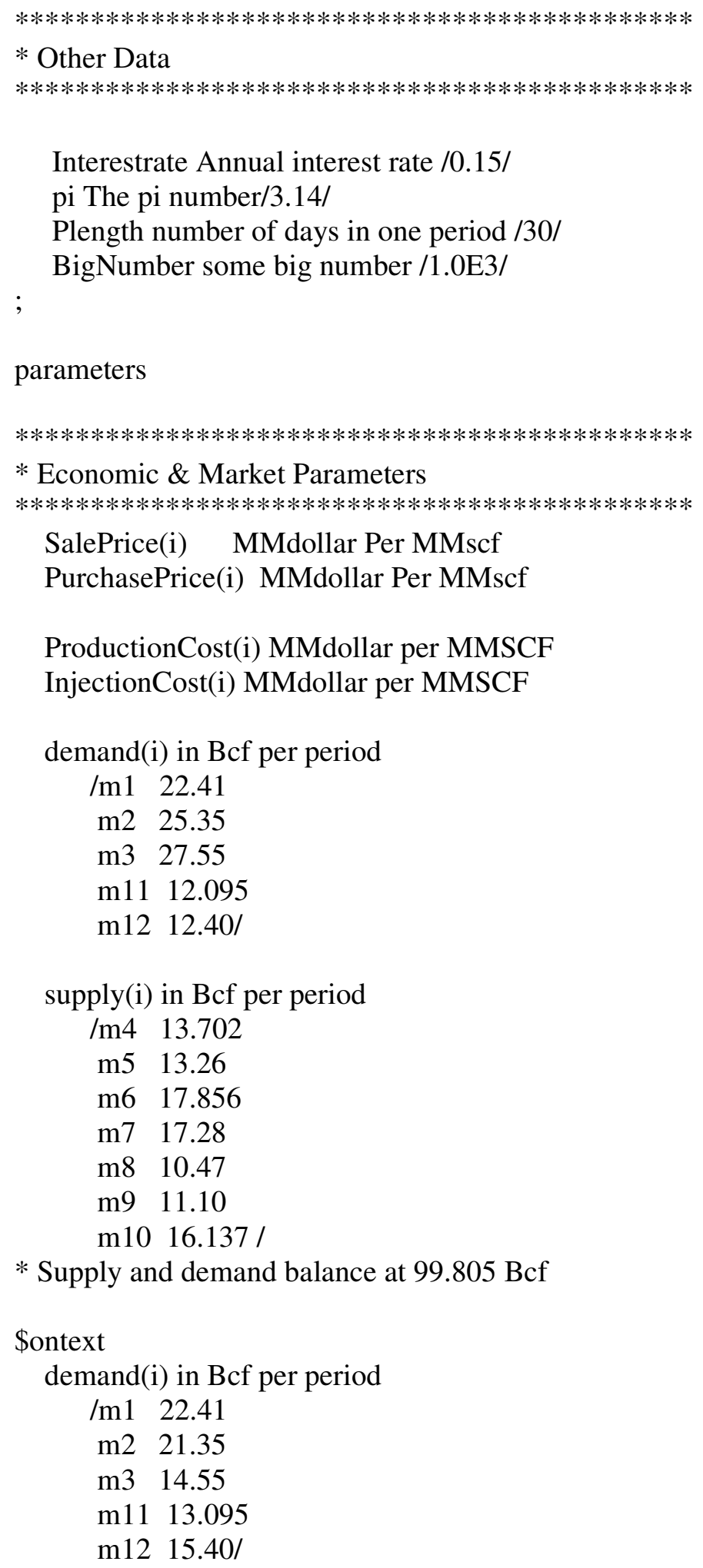

Interestrate Annual interest rate /0.15/

pi The pi number/3.14/

Plength number of days in one period $/ 30 /$

BigNumber some big number /1.0E3/

parameters

$* * * * * * * * * * * * * * * * * * * * * * * * * * * * * * * * * * * * * * * * * * * * * *$

* Economic \& Market Parameters

$* * * * * * * * * * * * * * * * * * * * * * * * * * * * * * * * * * * * * * * * * * * * *$

SalePrice(i) MMdollar Per MMscf

PurchasePrice(i) MMdollar Per MMscf

ProductionCost(i) MMdollar per MMSCF

InjectionCost(i) MMdollar per MMSCF

demand(i) in Bcf per period
/m1 22.41
$\mathrm{m} 2 \quad 25.35$
m3 27.55
m11 12.095
m12 12.40/

supply(i) in Bcf per period

/m4 13.702

m5 13.26

m6 17.856

m7 17.28

m8 10.47

m9 11.10

m10 16.137 /

* Supply and demand balance at $99.805 \mathrm{Bcf}$

\$ontext

demand(i) in Bcf per period
/m1 22.41
m2 21.35
m3 14.55
m11 13.095

m12 15.40/ 
supply(i) in Bcf per period

$$
\begin{array}{cl}
/ \mathrm{m} 4 & 13.702 \\
\text { m5 } & 13.26 \\
\text { m6 } & 17.856 \\
\text { m7 } & 17.28 \\
\text { m8 } & 11.47 \\
\text { m9 } & 11.10 \\
\text { m10 } & 2.137 /
\end{array}
$$

* Supply and demand balance at 8.6805*1E6 Mscf \$offtext

demandmean(i) in Bcf per period

$$
\begin{array}{cl}
/ \mathrm{m} 1 & 22.41 \\
\mathrm{~m} 2 & 25.35 \\
\mathrm{~m} 3 & 27.55 \\
\mathrm{~m} 11 & 12.095 \\
\mathrm{~m} 12 & 12.40 /
\end{array}
$$

supplymean(i) in Bcf per period

$$
\begin{array}{ll}
/ \mathrm{m} 4 & 13.702 \\
\text { m5 } & 13.26 \\
\text { m6 } & 17.856 \\
\text { m7 } & 17.28 \\
\text { m8 } & 10.47 \\
\text { m9 } & 11.10 \\
\text { m10 } & 16.137 /
\end{array}
$$

demandstd(i)

supplystd(i)

$\mathrm{W} \operatorname{cost}(\mathrm{W})$ in MMdollar per well

Wcostmean $(\mathrm{W})$

$\mathrm{W} \operatorname{coststd}(\mathrm{W})$

* Winjcost $(\mathrm{W})$ in Mdollar per well

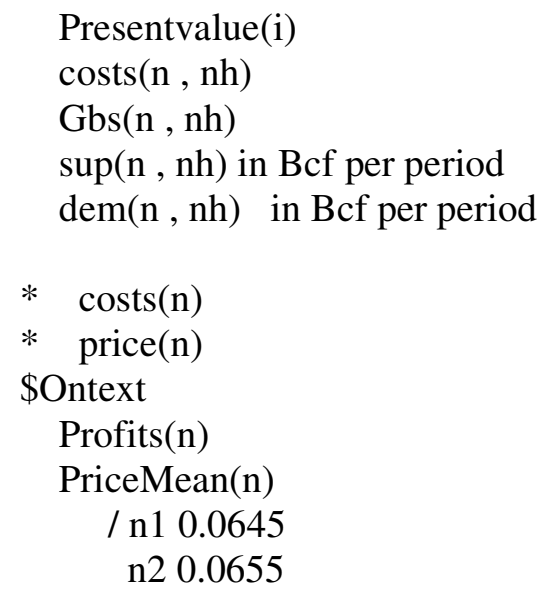




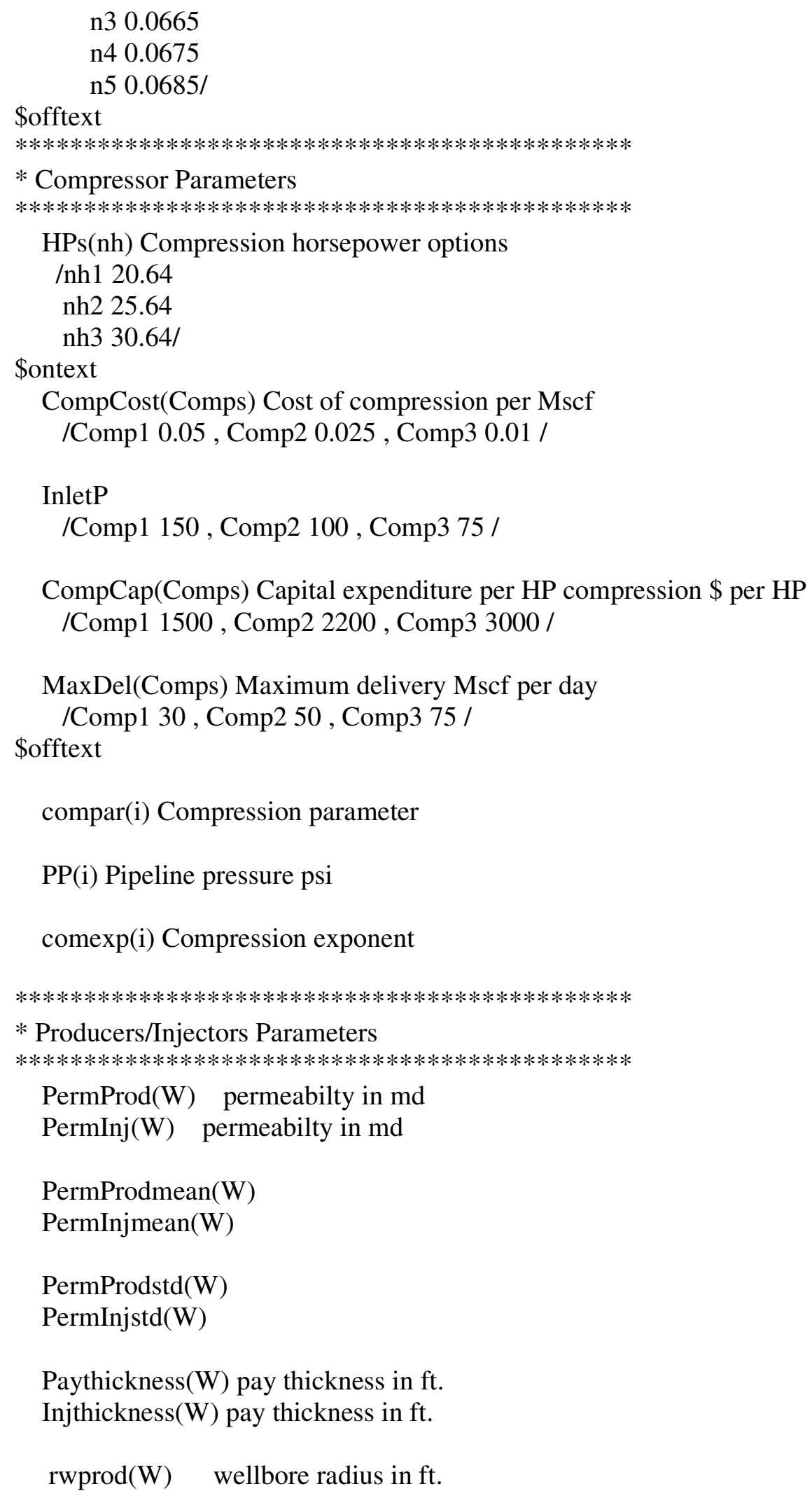


Area(W) Sweep area for each well in acres pex $(\mathrm{W})$ pressure exponent Jpro(W) Productivity Index of producers MMscf per MMpsi2

Jinj(W) Injectivity Index of injectors MMscf per MMpsi2

$* * * * * * * * * * * * * * * * * * * * * * * * * * * * * * * * * * * * * * * * * * * * * *$

* PVT Parameters

$* * * * * * * * * * * * * * * * * * * * * * * * * * * * * * * * * * * * * * * * * * * * *$

MuZpro(W) average(Viscosity*compression) factor in cp;

$* * * * * * * * * * * * * * * * * * * * * * * * * * * * * * * * * * * * * * * * * * * * * *$

* Parameters Definition

$* * * * * * * * * * * * * * * * * * * * * * * * * * * * * * * * * * * * * * * * * * * * *$

$* \quad \operatorname{price}(\mathrm{n})=3.5 * 1000 / 10 * * 6$;

* $\quad$ Saleprice $(\mathrm{i})=\operatorname{normal}(0.00645,0.0025) \$(\operatorname{coldmonths}(\mathrm{i}))+\operatorname{normal}(0.00645$, $0.0025) \$($ hotmonths(i));

PurchasePrice $(\mathrm{i})=2 * 3.5 * 1000 / 10 * * 6$;

$* \operatorname{normal}(0.0035,0.00195) \$(\operatorname{coldmonths}(\mathrm{i}))+\operatorname{normal}(0.0035$,

$0.00195) \$($ hotmonths(i));

ProductionCost $(\mathrm{i})=2 * 0.5 * 1000 / 10 * * 6$;

InjectionCost $(\mathrm{i})=2 * 0.5 * 1000 / 10 * * 6$;

demand (hotmonths $)=0$;

supply $($ coldmonths $)=0$;

demandstd(i) $=0.15 *$ demandmean $(\mathrm{i})$;

supplystd $(\mathrm{i})=0.15^{*} \operatorname{supplymean}(\mathrm{i})$;

$\mathrm{W} \cos t(\mathrm{~W})=4.0$;

$\mathrm{W} \operatorname{costmean}(\mathrm{W})=\mathrm{W} \cos t(\mathrm{~W})$;

$\mathrm{W} \operatorname{coststd}(\mathrm{W})=0.2 * \mathrm{~W} \operatorname{costmean}(\mathrm{W})$;

Presentvalue $(\mathrm{i})=\operatorname{power}((1+\operatorname{Interestrate} / 12),-\operatorname{ord}(\mathrm{i}))$;

$\operatorname{rwprod}(\mathrm{W})=\mathrm{rw}$

paythickness $(\mathrm{W})=\mathrm{h}$;

Injthickness $(\mathrm{W})=\mathrm{h}$;

$\operatorname{PermProd}(\mathrm{W})=\mathrm{k}$;

$\operatorname{PermInj}(\mathrm{W})=\mathrm{k}$;

PermProdmean $(\mathrm{W})=\mathrm{k}$;

PermInjmean $(\mathrm{W})=\mathrm{k}$; 


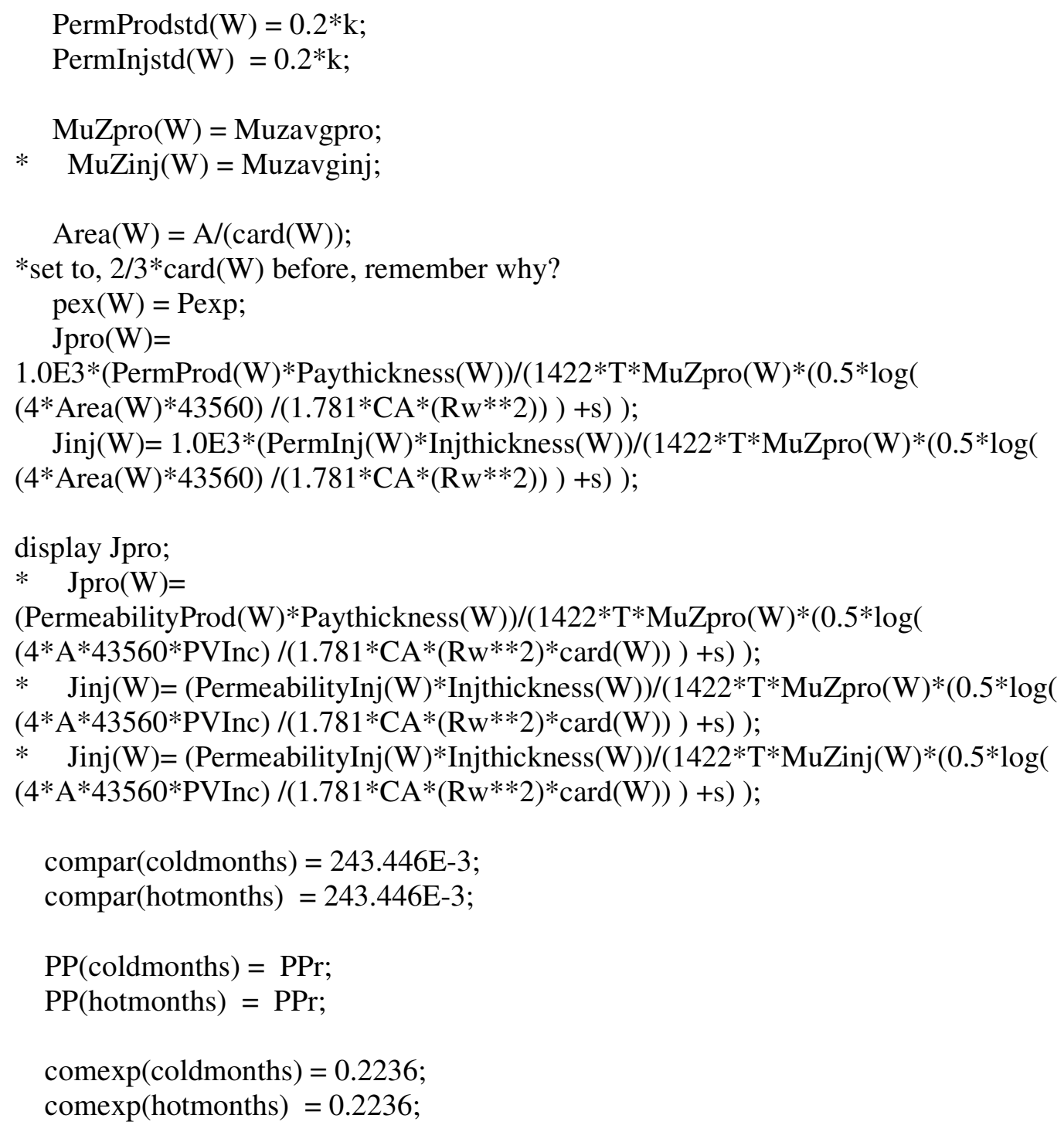

positive variables

$* * * * * * * * * * * * * * * * * * * * * * * * * * * * * * * * * * * * * * * * * * * * * *$

* Decision Variables

$* * * * * * * * * * * * * * * * * * * * * * * * * * * * * * * * * * * * * * * * * * * * * *$

qpro (i, W) production from an individual well for month i MMscf per day qinj $(i, W)$ Injection through an individual well for month i MMscf per day

* Gp(i) Cummilitive gas production by the end of month i Mscf

* Gi(i) Cummilitive gas Injection by the end of month i Mscf

PwfPro(i, W) Well flowing pressure from an individual well for month i Mpsi 
PInj(i) Injection pressure by an individual well for month i Mpsi

HP Total horsepower in MHP

* $\mathrm{n}$ (Comps) number of compressors to be installed from each type

$* * * * * * * * * * * * * * * * * * * * * * * * * * * * * * * * * * * * * * * * * * * * * *$

* Reservoir Variables

$* * * * * * * * * * * * * * * * * * * * * * * * * * * * * * * * * * * * * * * * * * * * *$

Pavg(i) Average Reservoir Pressure in Mpsi at the end of Period i

PZ(i) Average Pressure over $\mathrm{Z}$ as a function of pressure in Mpsi

PiZi Pi over Zi ratio at $\mathrm{i}=0$ in $\mathrm{Mpsi}$

Pinit Initial reservoir pressure (at $\mathrm{i}=0$ ) in Mpsi

Ginit Initial gas at $\mathrm{i}=0$ in Bcf

Gb Base gas in Bcf

* constcost;

binary variables

YWell(W) binary variable for potential well $\mathrm{j}$ drilled at $\mathrm{i}=0$;

Free variable

Cost Total investment and operation cost MM\$;

$* * * * * * * * * * * * * * * * * * * * * * * * * * * * * * * * * * * * * * * * * * * * *$
$*$ Initial Values
$* * * * * * * * * * * * * * * * * * * * * * * * * * * * * * * * * * * * * * * * * * * * *$

equations

Costeq

* Consisteq $1(\mathrm{~W})$

* Consisteq $2(\mathrm{~W})$

PZeq(i)

Piniteq

MBEeq(i)

Giniteq

Gfineq(i)

* Gbeq1(i)

Gbeq2

demandeq(i)

supplyeq(i) 
* Gpeq(i)

* Gieq(i)

Deliverabilityeq1 $(\mathrm{i}, \mathrm{W})$

* Deliverabilityeq2(i,W)

Injectivityeq(i, W)

mininjlimeq(i)

maxinjlimeq(i)

* minprolimeq $(\mathrm{i}, \mathrm{W})$

* Consistencyeq1(i, W)

* Consistencyeq2(i, W)

Consistencyeq(i, W)

Hpeq(i)

* testeq

;

$* * * * * * * * * * * * * * * * * * * * * * * * * * * * * * * * * * * * * * * * * * * * * *$

$*$ The Objective Function

$* * * * * * * * * * * * * * * * * * * * * * * * * * * * * * * * * * * * * * * * * * * * * *$

\section{Costeq... Cost $=\mathrm{e}=$}

$\operatorname{sum}((\mathrm{i})$,

- Presentvalue(i)*PurchasePrice(i)*Plength*(sum((W), qinj(i, W)))

+ Presentvalue $(\mathrm{i}) *$ PurchasePrice $(\mathrm{i}) *$ Plength* $(\operatorname{sum}((\mathrm{W})$, qinj $(\mathrm{i}, \mathrm{W})))$

+ Presentvalue $(\mathrm{i}) *$ ProductionCost $(\mathrm{i}) *$ Plength* $(\operatorname{sum}((\mathrm{W})$, qpro(i, W) $))$

+ Presentvalue $(\mathrm{i}) *$ InjectionCost(i)*Plength*(sum((W), qinj(i, W) ) ) )

$+\operatorname{sum}((\mathrm{W}), \mathrm{W} \operatorname{cost}(\mathrm{W}) * \mathrm{YWell}(\mathrm{W}))$

+ ComCost*HP

$+\mathrm{Gb}^{*} 1.0 \mathrm{E} 3 *$ PurchasePrice $(' \mathrm{~m} 1$ ');

* Presentvalue(i)*PurchasePrice(i)*Plength*(sum((W), qinj(i, W)))

$*$ Profit $=$ Presentvalue $(\mathrm{i}) *$ SalePrice $(\mathrm{i}) *(\operatorname{sum}((\mathrm{W})$, qpro $(\mathrm{i}, \mathrm{W})) *$ Plength $)$

$* * * * * * * * * * * * * * * * * * * * * * * * * * * * * * * * * * * * * * * * * * * * *$

* Reservoir Constraints

**************************************************

* Gpeq(i)..Gp(i) $=e=\mathrm{Gp}(\mathrm{i}-1) \$($ not ifirst $(\mathrm{i}))+\operatorname{sum}((\mathrm{W})$, qpro(i, W))*Plength;

* Gieq(i)..Gi(i) $=\mathrm{e}=\mathrm{Gi}(\mathrm{i}-1) \$($ not ifirst(i) $)+\operatorname{sum}((\mathrm{W})$, qinj(i, W $)) *$ Plength;

* Consisteq1(W)..sum((i), qpro(i , W )) =l= BigNumber* YWell(W);

* Consisteq2(W)..sum((i), qinj(i , W)) =l= BigNumber* YWell(W); 


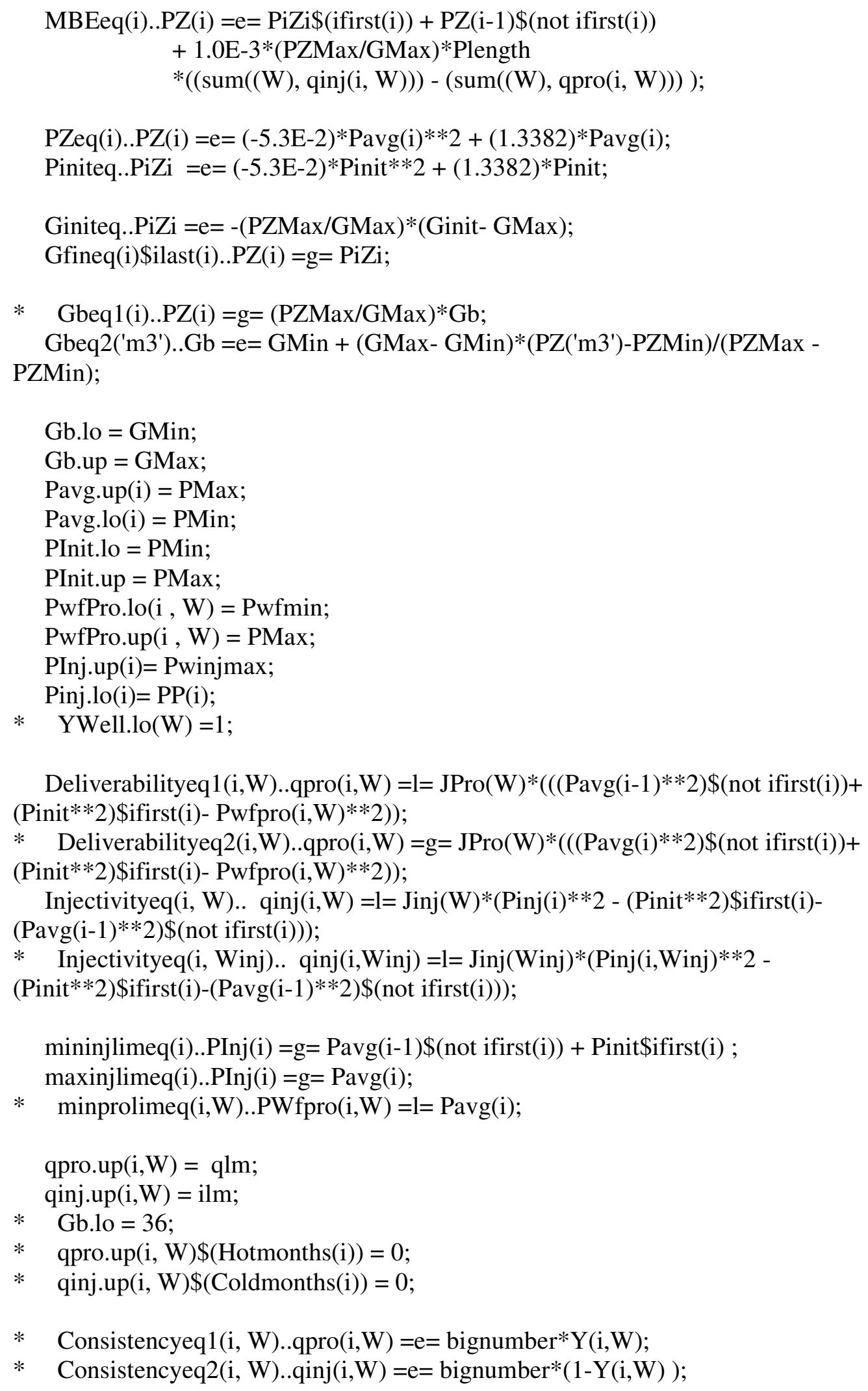

Deliverabilityeq1 $(\mathrm{i}, \mathrm{W})$. .qpro(i,W) $=\mathrm{l}=\mathrm{JPro}(\mathrm{W}) *(((\operatorname{Pavg}(\mathrm{i}-1) * * 2) \$($ not ifirst $(\mathrm{i}))+$ (Pinit**2)\$ifirst(i)- Pwfpro(i,W)**2));

* Deliverabilityeq2(i,W)..qpro $(\mathrm{i}, \mathrm{W})=\mathrm{g}=\mathrm{JPro}(\mathrm{W}) *((\operatorname{Pavg}(\mathrm{i}) * * 2) \$($ not ifirst $(\mathrm{i}))+$ (Pinit**2)\$ifirst(i)- Pwfpro(i,W)**2));

Injectivityeq(i, W).. qinj(i,W) $=\mathrm{l}=\operatorname{Jinj}(\mathrm{W})^{*}(\operatorname{Pinj}(\mathrm{i}) * * 2-($ Pinit**2)\$ifirst(i)$(\operatorname{Pavg}(\mathrm{i}-1) * * 2) \$($ not ifirst(i)));

* Injectivityeq(i, Winj).. qinj(i,Winj) $=1=\operatorname{Jinj}($ Winj $) *(\operatorname{Pinj}(i, W i n j) * * 2$ -

(Pinit**2)\$ifirst(i)-(Pavg(i-1)**2)\$(not ifirst(i)));

mininjlimeq(i)..PInj(i) $=\mathrm{g}=\operatorname{Pavg}(\mathrm{i}-1) \$($ not ifirst(i) $)+$ Pinit\$ifirst(i) ;

maxinjlimeq(i)..PInj(i) $=\mathrm{g}=\operatorname{Pavg}(\mathrm{i})$;

* $\quad$ minprolimeq(i,W)..PWfpro $(\mathrm{i}, \mathrm{W})=\mathrm{l}=\operatorname{Pavg}(\mathrm{i})$;

qpro.up $(\mathrm{i}, \mathrm{W})=$ qlm;

qinj.up $(\mathrm{i}, \mathrm{W})=\mathrm{ilm}$;

* Gb.lo = 36;

* $\quad$ qpro.up $(\mathrm{i}, \mathrm{W}) \$(\operatorname{Hotmonths}(\mathrm{i}))=0$;

* $\quad$ qinj.up $(\mathrm{i}, \mathrm{W}) \$($ Coldmonths $(\mathrm{i}))=0$;

* Consistencyeq1 $(\mathrm{i}, \mathrm{W}) . . q p r o(\mathrm{i}, \mathrm{W})=\mathrm{e}=$ bignumber* $\mathrm{Y}(\mathrm{i}, \mathrm{W})$;

* Consistencyeq2(i, W)..qinj $(\mathrm{i}, \mathrm{W})=\mathrm{e}=\operatorname{\operatorname {bignumber}} *(1-\mathrm{Y}(\mathrm{i}, \mathrm{W}))$; 
Consistencyeq(i, W)..qpro(i,W)*qinj(i,W) $=e=0$; W)

Hpeq(i)..HP $=\mathrm{g}=\operatorname{sum}((\mathrm{W}), 0.243446 *((\mathrm{PP}(\mathrm{i}) / \mathrm{Pwfpro}(\mathrm{i}, \mathrm{W})) * * 0.2236-1) * \mathrm{qpro}(\mathrm{i}$,

* $\quad$ Gb.lo = 36.839329;

* Hpeq(i , W)..HP(W) $=\mathrm{g}=$

compar(i)\$(coldmonths(i))*((PP(i)\$(coldmonths $(\mathrm{i})) / \mathrm{Pwfpro}(\mathrm{i}, \mathrm{W}))^{* *}$ comexp(i)\$(cold months(i)) - 1)*qpro(i , W)

*

$+\operatorname{compar}(\mathrm{i}) \$($ hotmonths(i) $) *((\operatorname{Pinj}(\mathrm{i}, \mathrm{W})) / \mathrm{PP}(\mathrm{i}) \$($ hotmonths(i) $) * * \operatorname{comexp}(\mathrm{i}) \$($ hotmonth s(i)) - 1)*qinj(i, W);

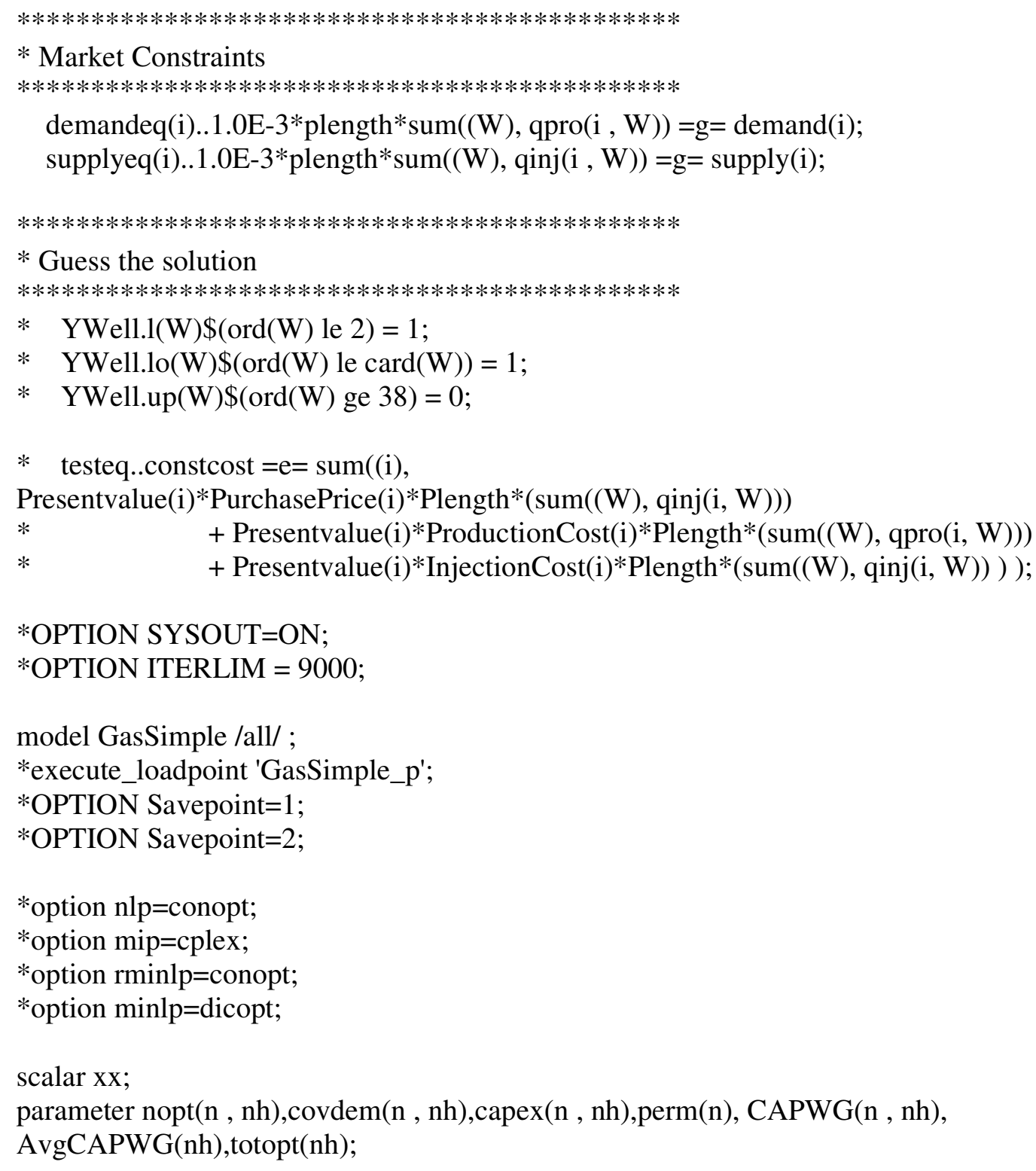


$\mathrm{xx}=$ GasSimple.Modelstat;

$\operatorname{nopt}(\mathrm{n}, \mathrm{nh})=0$;

totopt $(\mathrm{nh})=0$;

Display xx,GasSimple.Modelstat;

*solve GasSimple minimizing cost using MINLP ;

$\operatorname{loop}(\mathrm{n}$,

* $\quad \mathrm{W} \operatorname{cost}(\mathrm{W})=\operatorname{normal}(\mathrm{W} \operatorname{costmean}(\mathrm{W}), \mathrm{W} \operatorname{coststd}(\mathrm{W}))$;

$\operatorname{perm}(\mathrm{n})=\operatorname{normal}\left(\operatorname{Permprodmean}\left({ }^{\mathrm{W}} 11^{\prime}\right)\right.$, Permprodstd('W1'));

* $\quad \operatorname{normal}\left(\operatorname{Permprodmean}(\mathrm{W})-1.5^{*}\right.$ Permprodstd(W), Permprodstd(W));

$\operatorname{PermProd}(\mathrm{W})=\operatorname{perm}(\mathrm{n})$;

$\operatorname{PermInj}(\mathrm{W})=\operatorname{PermProd}(\mathrm{W})$;

$\operatorname{Jpro}(\mathrm{W})=$

$1.0 \mathrm{E} 3 *(\operatorname{PermProd}(\mathrm{W}) *$ Paythickness $(\mathrm{W})) /(1422 * \mathrm{~T} * \mathrm{MuZpro}(\mathrm{W}) *(0.5 * \log ($

$\left.\left.\left.(4 * \operatorname{Area}(\mathrm{W}) * 43560) /\left(1.781 * \mathrm{CA} *\left(\mathrm{Rw}^{* * 2}\right)\right)\right)+\mathrm{s}\right)\right)$;

$\operatorname{Jinj}(\mathrm{W})=$

$1.0 \mathrm{E} 3 *(\operatorname{PermInj}(\mathrm{W}) * \operatorname{Injthickness}(\mathrm{W})) /(1422 * \mathrm{~T} * \operatorname{MuZpro}(\mathrm{W}) *(0.5 * \log ($

$\left.\left.\left.(4 * \operatorname{Area}(\mathrm{W}) * 43560) /\left(1.781 * \mathrm{CA} *\left(\mathrm{Rw}^{*} * 2\right)\right)\right)+\mathrm{s}\right)\right)$;

demand(i) $\$(\operatorname{coldmonths}(\mathrm{i}))=\operatorname{normal}(\operatorname{demandmean}(\mathrm{i}), \operatorname{demandstd}(\mathrm{i}))$;

supply(i) $\$($ hotmonths(i) $=\operatorname{normal}(\operatorname{supplymean}(\mathrm{i}), \operatorname{supplystd}(\mathrm{i}))$;

$\operatorname{loop}(\mathrm{nh}$,

HP.lo = HPs(nh);

HP.up = HPs(nh);

* 4 is the standard deviation

execute_loadpoint 'GasSimple_p';

OPTION Savepoint $=1$;

* OPTION lp = cplex;

solve GasSimple minimizing cost using MINLP ;

If(GasSimple.Modelstat eq 2, Display 'Model terminate with normal

solution',GasSimple.Modelstat;

$\operatorname{nopt}(\mathrm{n}, \mathrm{nh})=1$;

totopt $(\mathrm{nh})=\operatorname{totopt}(\mathrm{nh})+1$;

$\operatorname{covdem}(\mathrm{n}, \mathrm{nh})=\operatorname{sum}((\mathrm{i}), \operatorname{demand}(\mathrm{i}))$;

$\operatorname{Gbs}(\mathrm{n}, \mathrm{nh})=\mathrm{Gb} .1$;

$\operatorname{capex}(\mathrm{n}, \mathrm{nh})=\operatorname{sum}((\mathrm{W}), \mathrm{W} \operatorname{cost}(\mathrm{W}))+\operatorname{ComCost} * \mathrm{HP} .1+$

Gb.1*1.0E3*PurchasePrice('m1');

CAPWG $(\mathrm{n}, \mathrm{nh})=\operatorname{capex}(\mathrm{n}, \mathrm{nh}) / \operatorname{covdem}(\mathrm{n}, \mathrm{nh}) ;$;

$\operatorname{costs}(\mathrm{n}, \mathrm{nh})=$ cost. 1 ;

$\operatorname{dem}(\mathrm{n}, \mathrm{nh})=\operatorname{sum}((\mathrm{i}), \operatorname{demand}(\mathrm{i}))$;

)

$\sup (\mathrm{n}, \mathrm{nh})=\operatorname{sum}((\mathrm{i}), \operatorname{supply}(\mathrm{i}))$;

) ;

$\operatorname{AvgCAPWG}($ nh) $\$(\operatorname{totopt}(\mathrm{nh})$ ge 1)= sum((n), CAPWG(n ,nh))/totopt(nh); 


\section{\$Ontext}

loop (n,

execute_loadpoint 'GasSimple_p';

OPTION Savepoint=1;

solve GasSimple minimizing cost using MINLP ;

If(GasSimple.Modelstat eq 2, Display 'Model terminate with normal

solution',GasSimple.Modelstat;

* $\operatorname{nopt}(\mathrm{n})=1$;

covdem $(n)=\operatorname{sum}((\mathrm{i}), \operatorname{demand}(\mathrm{i}))$;

$\operatorname{capex}(n)=\operatorname{sum}((\mathrm{W}), \mathrm{W} \operatorname{cost}(\mathrm{W}))+$ ComCost*HP.1 +

Gb.1*1.0E3*PurchasePrice('m1');

CAPWG $(n)=\operatorname{capex}(n) / \operatorname{covdem}(n) ;) ;$

) ;

\$offtext

$* * * * * * * * * * * * * * * * * * * * * * * * * * * * * * * * * * * * * * * * * * * * *$

* Excel Output

$* * * * * * * * * * * * * * * * * * * * * * * * * * * * * * * * * * * * * * * * * * * * * *$

execute_unload "results_V4-5.gdx" covdem,capex,CAPWG,AvgCAPWG, costs,nopt,totopt,covdem,Gbs,perm,Wcost;

*execute_unload "results_V4-5.gdx" covdem,capex,CAPWG,

costs,dem,sup,nopt,totopt,covdem,Gbs;

*execute_unload "results_V4-5.gdx" covdem,capex,CAPWG, covdem, cost.1,

YWell.1, HP.1, Pinit.1, Gb.1, qpro.1, qinj.1, Pavg.l, Pwfpro.1, Pinj.1;

*execute_unload "results_V6-1.gdx" costs,dem,sup,nopt,covdem,capex,CAPWG;

*execute 'gdxxrw.exe GasSimple_p.gdx';

*execute 'gdxxrw.exe results_V2.gdx var=Gp.L rng=Test!a3';

*execute 'gdxxrw.exe results_V2.gdx var=Gi.l rng=Test!a5'; 


\section{Nomenclature}

$$
\begin{aligned}
& A \quad=\text { Area, } \mathrm{ft}^{2} \\
& B_{g} \quad=\text { Gas formation volume factor, } \mathrm{RB} / \mathrm{Mscf} \\
& B_{g i} \quad=\text { Initial gas formation volume factor, RB/Mscf } \\
& B_{w} \quad=\text { Water formation volume factor, } \mathrm{RB} / \mathrm{STB} \\
& c_{w} \quad=\text { Water compressibility factor } \mathrm{psi}^{-1} \\
& c_{f} \quad=\text { Formation compressibility factor } \mathrm{psi}^{-1} \\
& C_{A} \quad=\text { Shape factor } \\
& G \quad=\text { Original gas in place, Mscf } \\
& G_{I} \quad=\text { Cumulative gas injection, Mscf } \\
& G_{p} \quad=\text { Cumulative gas production, Mscf } \\
& h \quad=\text { Reservoir thickness, } \mathrm{ft} \\
& \text { HP = Total compression horsepower, } \mathrm{MHP} \\
& H P_{i n j} \quad=\text { Compression horsepower for injection, MHP } \\
& H P_{\text {pro }}=\text { Compression horsepower for production, } \mathrm{MHP} \\
& J_{k} \quad=\text { Productivity index for well } \mathrm{k}, \mathrm{Mscf} / \mathrm{psi}^{2} \\
& k \quad=\text { Permeability, md } \\
& n \quad=\text { Pressure exponent } \\
& \bar{P} \quad \text { = Average reservoir pressure, psi } \\
& P_{i} \quad=\text { Initial reservoir pressure, } \mathrm{psi} \\
& P_{\text {pipeline }} \quad=\text { Pipeline pressure, } \mathrm{psi} \\
& P_{w f, k} \quad=\text { Well flowing pressure for well k, psi } \\
& P_{w h} \quad=\text { Wellhead pressure, } \mathrm{psi} \\
& q_{k} \quad=\text { Production from well k, Mscf/day } \\
& Q_{i n j, t o t}=\text { Total injection rate, Mscf/D } \\
& Q_{\text {pro,tot }}=\text { Total production rate, Mscf/D } \\
& r_{w, k} \quad=\text { Wellbore radius for well } \mathrm{k}, \mathrm{ft}
\end{aligned}
$$




$\begin{array}{ll}s_{k} & =\text { Skin factor for well } \mathrm{k} \\ T & =\text { Temperature, }{ }^{\mathrm{o}} \mathrm{R} \\ W_{p} & =\text { Cumulative water produced, STB } \\ W_{I} & =\text { Cumulative water injected, STB } \\ W_{e} & =\text { Cumulative water influx, } \mathrm{RB} \\ Z & =\text { Gas deviation factor } \\ Z_{i} & =\text { Gas deviation factor at initial pressure } \\ \mu_{g} & =\text { Gas viscosity, } \mathrm{cp} \\ \Gamma & =\text { Transmissibility factor for a layer/compartment, } \mathrm{md} . \mathrm{ft}^{2} / \mathrm{cp} \\ \Gamma_{12} & =\text { Transmissibility between layers/compartments } 1 \& 2, \mathrm{md} . \mathrm{ft}^{2} / \mathrm{cp} \\ \Delta P & =\text { Reservoir pressure change, } \mathrm{psi}\end{array}$




\section{References}

Ahmed, T. and McKinney, P.D., 2005. Advanced Reservoir Engineering. Elsevier. Oxford. UK.

Bennion, D., Thomas, F., Ma, T. and Imer, D. 2000. Detailed Protocol for the Screening and Selection of Gas Storage Reservoirs. Paper SPE 59738 presented at the 2000 SPE/CERI Gas Technology Symposium, Calgary, Alberta Canada, 3-5 April.

Bilgesu, H. and Ali, W. 2004. Effect of Reservoir Properties on the Performance and Design of Gas Storage Wells. Paper SPE 91441 presented at the SPE Eastern Regional Meeting, Charleston, WV, 15-17 September.

Bratvold, R.B., Bickel, E., and Lohne, H.P., 2007. Value of Information in Oil and Gas Industry: Past, Present, and Future. Paper SPE 110378. Presented at the SPE Annual Technical Confrence and Exhabition, Anaheim, California, 11-14 November.

Brown, K.G. and Sawyer, W.K., 1999. Practical Methods to Improve Storage Operations - A Case Study. paper SPE 57460. presented at the SPE Eastern Regional Conference and Exhibition held in Charleston, West Virginia, 20-22 October 1999.

Carlos, A. and Chu-Jon, B.S., 1973. Simulation of a Gas Storage Reservoir System. Masters report, The U. of Texas at Austin, Austin, Texas.

Coats, K.H., 1969. Use and Misuse of Reservoir Simulation Models. JPT, November: 1391-1398. SPE 02367.

Coats, K. 1969. An Approach to Locating New Wells in Heterogeneous Gas Producing Fields. J. Pet Tech (May): 549-558.

De Moegen, H., Nabil, R., Lehuen, P., and Sonier, F. 1996. Well-Surface Connection Controls for Gas Reservoir Simulation. Paper SPE 35625 presented at the SPE Gas Technology Conference, Calgary, Alberta Canada, 28 April - 1 May.

Duane, J. 1967. Gas Storage Field Development Optimization. J. Pet Tech (March): 323-330.

Economides M., Hill, A. and Ehlig-Economides, C. 1994. Petroleum Production Systems. PTR Prentice Hall Inc., Englewood Cliffs, NJ.

EIA. 2004. The Basics of Underground Natural Gas Storage. Energy Information Administration (August).

EIA. 2006. US Underground Natural Gas Storage Developments: 1998-2005. Energy Information Administration (October).

Energy Information Administration. (2009, September 24). Energy Information Administration. Retrieved September 26, 2009, from Weekly Natural Gas Storage Report: http://www.eia.doe.gov/oil_gas/natural_gas/ngs/ngs.html 
FERC. 2004. Current State of and Issues Concerning Underground Natural Gas Storage. Federal Energy Regulatory Commission, 30 September.

Hagoort, J. and Hoogstra, R., 1999. Numerical solution of the material balance equations of compartmented gas reservoirs. SPE Reservoir Eng., 2, (August).

Haines, L. 2009. Plenty of Gas In Store. Oil and Gas Investor (June).

Henderson, J., Dempsey, J., and Tyler, J. 1968. Use of Numerical Models to Develop and Operate Gas Storage Reservoirs. J. Pet Tech (November): 1239-1246.

Hosgor, E. 2009. Assessing the Impact of Commodity Price Assumptions on Facility Optimization: A Case in Offshore Oil and Gas. MS Thesis, The University of Texas at Austin, Austin, TX.

Hewer, T.L., Fugate, M.W. and Owens, R.W., 1993. Improved Performance in Aquifer Gas Storage Fields Through Reservoir Management. Paper SPE 26172. Presented at SPE Gas Technology Symposiom, Calgary, Alberta, Canada, 28-30 June.

Hultzsch, P., Lake, Larry W., and Gilbert, R. 2007. Decision and Risk Analysis Through the Life of the Field. Paper SPE 107704 presented at the SPE Hydrocarbon Economics Evaluation Symposium, Dallas, Texas, 1-3 April.

Jablonowski, C., Wiboonkij-Arphakul, C., and Neuhold, M. 2008. Estimating the Cost of Errors in Estimates Used During Concept Selection. SPE Proj Fac \& Const, 3 (1): 1-6.

Jankovic, I., Fiori, A., and Dagan, G. 2006. Modeling Flow and Transport in Highly Heterogeneous Three-Dimensional Aquifers: Ergodicity, Gaussianity, and Anomolous Behavior: Conceptual Issues and Numerical Simulations. Water Resources Research, 42.

Johnson, V., Ammer, J. and Trick, M. 2000. Improving Gas Storage Development Planning Through Simulation-Optimization. Paper SPE 65639 presented at the SPE Eastern Regional Meeting in Morgantown, WV, 17-19 October.

Khodri, D., Lehuen, P., Labaune, B., and Nabil, R. 1997. Modeling of Reservoir to Surface Network Coupling: A Case Study of Gas Storage Management. Paper SPE 38864 presented at the SPE Annual Technical Conference and Exhibition, San Antonio, Texas, 5-8 October.

Kuncir, M., Chang, J., Mansdorfer, J. and Dougherty, E. 2003. Analysis and Optimal Design of Gas Storage Reservoirs. Paper SPE 84822 presented at the SPE Eastern Regional/AAPG Eastern Section Joint Meeting, Pittsburgh, PA, 6-10 September.

Lawal, A., Srinivasan, S., and Lake, Larry W. 2008. Sensitivity Analyses of Production and Recovery Forecasts Using Variance-Based Methods. Paper SPE 110846 presented at the SPE Western Regional and Pacific Section AAPG Joint Meeting, Bakersfield, CA, 29 March-2 April. 
Macary, S.M., Hassan, A., and Ragaee, E., 1999. Better Understanding of Reservoir Statistics is the Key for Reliable Monte Carlo Simulation. Ppaer SPE 53264, presented at the SPE Middle East Oil Show, Bahrain, 20-23 February.

McVay, D. and Spivey, J. 2001. Optimizing Gas-Storage Reservoir Performance. SPE Res Eval \& Eng (June): 173-178.

McFarland, J. W., Lasdon L. and Loose V., March-April, 1984. Development Planning and Management of Petroleum Reservoirs Using Tank Models and Nonlinear Programming. Operations Research, Vol. 32, No. 2. 270-289.

NaturalGas.org. (n.d.). Storage of Natural Gas. Retrieved March 19, 2009, from NaturalGas.org: http://www.naturalgas.org/naturalgas/storage.asp

Odeh, A. 1969. Reservoir Simulation: What is it? J. Pet Tech (November): 1383-1388.

Odeh, A. 1982. An Overview of Mathematical Modeling of the Behavior of Hydrocarbon Reservoirs. SIAM Review, 24 (3): 263-273.

Payne, D.A., 1996. Material balance calculations in tight gas reservoirs: the pitfalls of $\mathrm{p} / \mathrm{Z}$ plots and a more accurate technique. SPE Reservoir Eng., November.

Purwar, S. 2008. A Study on the Use of Proxy Reservoir Models in Field Development Optimization and Value of Information Problems. MS Thesis, The University of Texas at Austin, Austin, TX.

Van Horn, H. and Wienecke, D. 1970. A Method for Optimizing the Design of Gas Storage Systems. Paper SPE 2966 presented at the SPE Annual Meeting, Houston, Texas, 4-7 October.

The Federal Energy Regulatory Commission. (n.d.). FERC: Blanket Certificates. Retrieved July 20, 2009, from www.ferc.gov:

http://www.ferc.gov/industries/gas/indus-act/blank-cert.asp\#skipnavsub

Wells, J.A. and Evans, L.J., 1992. Engineering Evaluation and Performance Analysis of the Loop Gas Storage Field. paper SPE 24922 presented at the SPE Annual Technical Conference and Exhibition, Washington, D.C., 4-7 October.

Walsh, M. and Lake, Larry W. 2003. A Generalized Approach to Primary Hydrocarbon Recovery Of Petroleum Exploration \& Production. Elsevier Science B.V., Amsterdam, The Netherlands.

Wattenbarger, R. 1970. Maximizing Seasonal Withdrawals From Gas Storage Reservoirs. J. Pet Tech (August): 994-998. 


\section{Vita}

Amin Ettehadtavakkol was born in Esfahan, Iran in February, 1984. After completing his high school studies in 2002, he entered Sharif University of Technology, Tehran, Iran. In September 2007, he graduated with double BS degrees in Petroleum Engineering and Industrial Engineering. In June 2008, he entered the Graduate School of the University of Texas at Austin and began his Masters in the Department of Petroleum and Geosystems Engineering. Upon completion of his MS studies in 2009, he will commence his PhD studies in the area of integrated reservoir-economic analysis and E\&P project optimization.

Permanent address:

3569 Lake Austin Blvd,

Austin, Texas

This thesis was typed by the author. 